\title{
Surgical fixation methods for tibial plateau fractures (Review)
}

\author{
McNamara IR, Smith TO, Shepherd KL, Clark AB, Nielsen DM, Donell S, Hing CB
}

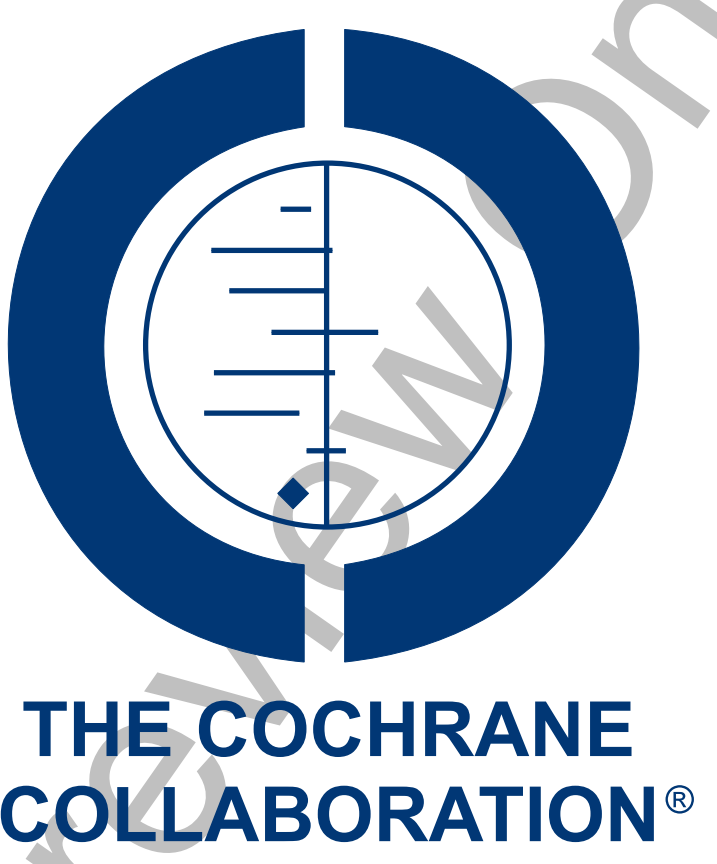

This is a reprint of a Cochrane review, prepared and maintained by The Cochrane Collaboration and published in The Cochrane Library 2015, Issue 9

http://www.thecochranelibrary.com

\section{WILEY}

Surgical fixation methods for tibial plateau fractures (Review)

Copyright @ 2015 The Cochrane Collaboration. Published by John Wiley \& Sons, Ltd. 
TABLE OF CONTENTS

HEADER . . . . . . . . . . . . . . . . . . . . . . . . . . . . . . . . . . . . . . . . . . . . . . . . . . . .

ABSTRACT . . . . . . . . . . . . . . . . . . . . . . . . . . . . . . . . . . . . . . . . . . . . . . . . . .

PLAIN LANGUAGE SUMMARY . . . . . . . . . . . . . . . . . . . . . . . . . . . . . . . . . . . . . . . . . . . . . .

SUMMARY OF FINDINGS FOR THE MAIN COMPARISON $\quad . \quad$. . . . . . . . . . . . . . . . . . . . . . . . . . .

BACKGROUND . . . . . . . . . . . . . . . . . . . . . . . . . . . . . . . . . . . . . . . . . . . . . . . . . . .

OBJECTIVES . . . . . . . . . . . . . . . . . . . . . . . . . . . . . . . . . . . . . . . . . . .

METHODS . . . . . . . . . . . . . . . . . . . . . . . . . . . . . . . . . . . . . . . . . . 99

RESULTS . . . . . . . . . . . . . . . . . . . . . . . . . . . . . . . . . . . . . . . 11

Figure 1. . . . . . . . . . . . . . . . . . . . . . . . . . . . . . . . . . . . . . . 13

Figure 2. . . . . . . . . . . . . . . . . . . . . . . . . . . . . . . . . . . . . . 16

Figure 3. . . . . . . . . . . . . . . . . . . . . . . . . . . . . . . . . . . . 17

DISCUSSION . . . . . . . . . . . . . . . . . . . . . . . . . . . . . . . . . . . . . . . . . . . . .

AUTHORS' CONCLUSIONS . . . . . . . . . . . . . . . . . . . . . . . . . . . . . . . . . 24

ACKNOWLEDGEMENTS . . . . . . . . . . . . . . . . . . . . . . . . . . . . . . . . . . . . . . . . . .

REFERENCES . . . . . . . . . . . . . . . . . . . . . . . . . . . . . . . . . . . . . . . .

CHARACTERISTICS OF STUDIES . . . . . . . . . . . . . . . c . . . . . . . . . . . . . . . . . . . . . . . . .

DATA AND ANALYSES . . . . . . . . . . . . . . . . . . . . . . . . . . . . . . . . . . . . . . . . . . . . . 45

Analysis 1.1. Comparison 1 Circular external fixation with limited internal fixation (hybrid fixation) versus standard open reduction internal fixation, Outcome 1 SF-36 scores at 24 months. . . . . . . . . . . . . . . . . . . . . .

Analysis 1.2. Comparison 1 Circular external fixation with limited internal fixation (hybrid fixation) versus standard open reduction internal fixation, Outcome 2 Hospital for Special Surgery Score. . . . . . . . . . . . . . .

Analysis 1.3. Comparison 1 Circular external fixation with limited internal fixation (hybrid fixation) versus standard open reduction internal fixation, Outcome 3 WOMAC Pain score.

Analysis 1.4. Comparison 1 Circular external fixation with limited internal fixation (hybrid fixation) versus standard open reduction internal fixation, Outcome 4 WOMAC Stiffness score. . . . . . . . . . . . . . . . . . . . . .

Analysis 1.5. Comparison 1 Circular external fixation with limited internal fixation (hybrid fixation) versus standard open reduction internal fixation, Outcome 5 WOMAC Function score. . . . . . . . . . . . . . . . . . .

Analysis 1.6. Comparison 1 Circular external fixation with limited internal fixation (hybrid fixation) versus standard open reduction internal fixation, Outcome 6 Reoperations (24 months).

Analysis 1.7. Comparison 1 Circular external fixation with limited internal fixation (hybrid fixation) versus standard open reduction internal fixation, Outcome 7 Intra-operative measures (length of surgery, blood loss). . . . . . .

Analysis 1.8. Comparison 1 Circular external fixation with limited internal fixation (hybrid fixation) versus standard open reduction internal fixation, Outcome 8 Knee joint total range of motion (degrees) at 24 months. . . . . . .

Analysis 1.9. Comparison 1 Circular external fixation with limited internal fixation (hybrid fixation) versus standard open reduction internal fixation, Outcome 9 Return to pre-injury activity level. . . . . . . . . . . . . . . . . . .

Analysis 1.10. Comparison 1 Circular external fixation with limited internal fixation (hybrid fixation) versus standard open reduction internal fixation, Outcome 10 Length of hospital stay (days). . . . . . . . . . . . . . . . 56

Analysis 1.11. Comparison 1 Circular external fixation with limited internal fixation (hybrid fixation) versus standard open reduction internal fixation, Outcome 11 Radiological outcomes (postoperatively). . . . . . . . . . . . . . . 57

Analysis 1.12. Comparison 1 Circular external fixation with limited internal fixation (hybrid fixation) versus standard open reduction internal fixation, Outcome 12 Radiological evidence of tibiofemoral joint osteoarthritis. . . . . .

Analysis 2.1. Comparison 2 Less invasive stabilisation system (LISS) versus double-plating open reduction internal fixation, Outcome 1 Hospital for Special Surgery Score.

Analysis 2.2. Comparison 2 Less invasive stabilisation system (LISS) versus double-plating open reduction internal fixation, Outcome 2 Complications (24 months).

Analysis 2.3. Comparison 2 Less invasive stabilisation system (LISS) versus double-plating open reduction internal fixation, Outcome 3 Reoperations (up to 24 months).

Analysis 2.4. Comparison 2 Less invasive stabilisation system (LISS) versus double-plating open reduction internal fixation,

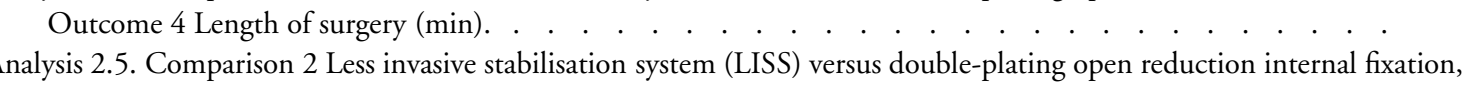
Outcome 5 Incision length $(\mathrm{cm})$. 
Analysis 2.6. Comparison 2 Less invasive stabilisation system (LISS) versus double-plating open reduction internal fixation, Outcome 6 Perioperative blood loss $(\mathrm{ml})$.

Analysis 2.7. Comparison 2 Less invasive stabilisation system (LISS) versus double-plating open reduction internal fixation, Outcome 7 Postsurgical malreduction and malalignment. . . . . . . . . . . . . . . . . . . . . . . .

Analysis 2.8. Comparison 2 Less invasive stabilisation system (LISS) versus double-plating open reduction internal fixation, Outcome 8 Time to radiological union (weeks).

Analysis 3.1. Comparison 3 Arthroscopically assisted percutaneous reduction and internal fixation versus standard open reduction and internal fixation, Outcome 1 Hospital for Special Surgery Score (14 months).

Analysis 3.2. Comparison 3 Arthroscopically assisted percutaneous reduction and internal fixation versus standard open reduction and internal fixation, Outcome 2 Knee stiffness.

Analysis 3.3. Comparison 3 Arthroscopically assisted percutaneous reduction and internal fixation versus standard open reduction and internal fixation, Outcome 3 Length of surgery $(\mathrm{min})$. . . . . . . . . . . . . . . . . . .

Analysis 3.4. Comparison 3 Arthroscopically assisted percutaneous reduction and internal fixation versus standard open reduction and internal fixation, Outcome 4 Incision length $(\mathrm{cm})$.

Analysis 3.5. Comparison 3 Arthroscopically assisted percutaneous reduction and internal fixation versus standard open reduction and internal fixation, Outcome 5 Knee joint range of motion (degrees) (14 months). . . . . . .

Analysis 3.6. Comparison 3 Arthroscopically assisted percutaneous reduction and internal fixation versus standard open reduction and internal fixation, Outcome 6 Time to radiological union (months). . . . . . . . . . . . .

Analysis 4.1. Comparison 4 Bone substitute versus autologous bone graft, Outcome 1 Lower limb function. . . .

Analysis 4.2. Comparison 4 Bone substitute versus autologous bone graft, Outcome 2 Adverse events. . . . . . .

Analysis 4.3. Comparison 4 Bone substitute versus autologous bone graft, Outcome 3 Range of movement (at 12 months).

Analysis 4.4. Comparison 4 Bone substitute versus autologous bone graft, Outcome 4 Radiographic outcomes. . .

Analysis 4.5. Comparison 4 Bone substitute versus autologous bone graft, Outcome 5 Anatomical malalignment.

APPENDICES

.. . . . . . . . . . . . . . . . 70

CONTRIBUTIONS OF AUTHORS . . . . . . . . . . . . . . . . . . . . . . . . . . . . . . . . . . . . . . . . .

DECLARATIONS OF INTEREST . . . . . . . . . . . . . . . . . . . . . . . . . . . . . . . . 73

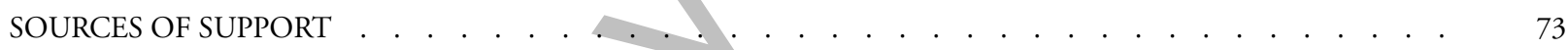

DIFFERENCES BETWEEN PROTOCOL AND REVIEW . . . . . . . . . . . . . . . . . . . . . . . . . 73

Surgical fixation methods for tibial plateau fractures (Review) 


\title{
Surgical fixation methods for tibial plateau fractures
}

\author{
Iain R McNamara ${ }^{1}$, Toby O Smith ${ }^{2}$, Karen L Shepherd ${ }^{1}$, Allan B Clark ${ }^{2}$, Dominic M Nielsen ${ }^{3}$, Simon Donell ${ }^{2}$, Caroline B Hing ${ }^{3}$ \\ ${ }^{1}$ Department of Trauma and Orthopaedics, Norfolk and Norwich University NHS Trust, Norwich, UK. ${ }^{2}$ Faculty of Medicine and \\ Health Sciences, University of East Anglia, Norwich, UK. ${ }^{3}$ Department of Trauma and Orthopaedic Surgery, St George's Hospital, \\ London, UK
}

Contact address: Iain R McNamara, Department of Trauma and Orthopaedics, Norfolk and Norwich University NHS Trust, Norfolk and Norwich University Hospital, Colney Lane, Norwich, Norfolk, NR4 7UY, UK. iain.mcnamara@nnuh.nhs.uk.

Editorial group: Cochrane Bone, Joint and Muscle Trauma Group.

Publication status and date: New, published in Issue 9, 2015.

Review content assessed as up-to-date: 2 September 2014.

Citation: McNamara IR, Smith TO, Shepherd KL, Clark AB, Nielsen DM, Donell S, Hing CB. Surgical fixation methods for tibial plateau fractures. Cochrane Database of Systematic Reviews 2015, Issue 9. Art. No.: CD009679. DOI: 10.1002/14651858.CD009679.pub2.

Copyright (C) 2015 The Cochrane Collaboration. Published by John Wiley \& Sons, Ltd.

\section{Background}

\section{A B S T R A C T}

Fractures of the tibial plateau, which are intra-articular injuries of the knee joint, are often difficult to treat and have a high complication rate, including early-onset osteoarthritis. Surgical fixation is usually used for more complex tibial plateau fractures. Additionally, bone void fillers are often used to address bone defects caused by the injury. Currently there is no consensus on either the best method of fixation or bone void filler.

\section{Objectives}

To assess the effects (benefits and harms) of different surgical interventions, and the use of bone void fillers, for treating tibial plateau fractures.

\section{Search methods}

We searched the Cochrane Bone, Joint and Muscle Trauma Group Specialised Register (12 September 2014), the Cochrane Central Register of Controlled Trials (2014 Issue 8), MEDLINE (1946 to September Week 1 2014), EMBASE (1974 to 2014 Week 36), trial registries (4 July 2014), conference proceedings and grey literature (4 July 2014).

\section{Selection criteria}

We included randomised and quasi-randomised controlled clinical trials comparing surgical interventions for treating tibial plateau fractures and the different types of filler for filling bone defects.

\section{Data collection and analysis}

Two review authors independently screened search results, selected studies, extracted data and assessed risk of bias. We calculated risk ratios (RR) for dichotomous outcomes and mean differences (MD) for continuous outcomes, with 95\% confidence intervals (CIs). Only very limited pooling, using the fixed-effect model, was possible. Our primary outcomes were quality of life measures, patientreported outcome measures of lower limb function and serious adverse events.

Surgical fixation methods for tibial plateau fractures (Review)

Copyright $\odot 2015$ The Cochrane Collaboration. Published by John Wiley \& Sons, Ltd. 


\section{Main results}

We included six trials in the review, with a total of 429 adult participants, the majority of whom were male (63\%). Three trials evaluated different types of fixation and three analysed different types of bone graft substitutes. All six trials were small and at substantial risk of bias. We judged the quality of most of the available evidence to be very low, meaning that we are very uncertain about these results.

One trial compared the use of a circular fixator combined with insertion of percutaneous screws (hybrid fixation) versus standard open reduction and internal fixation (ORIF) in people with open or closed Schatzker types V or VI tibial plateau fractures. Results (66 participants) for quality of life scores using the 36-item Short Form Health Survey (SF-36)), Hospital for Special Surgery (HSS) scores and Western Ontario and McMaster Universities Osteoarthritis Index (WOMAC) function scores tended to favour hybrid fixation, but a benefit of ORIF could not be ruled out. Participants in the hybrid fixation group had a lower risk for an unplanned reoperation (351 per 1000 people compared with 450 in the ORIF group; 95\% CI 197 fewer to 144 more) and were more likely to have returned to their pre-injury activity level (303 per 1000 people, compared with 121 in the ORIF group; 95\% CI 15 fewer to 748 more). Results of the two groups were comparable for the WOMAC pain subscale and stiffness scores, but mean knee range of motion values were higher in the hybrid group.

Another trial compared the use of a minimally invasive plate (LISS system) versus double-plating ORIF in 84 people who had open or closed bicondylar tibial plateau fractures. Nearly twice as many participants (22 versus 12 ) in the ORIF group had a bone graft. Quality of life, pain, knee range of motion and return to pre-injury activity were not reported. The trial provided no evidence of differences in HSS knee scores, complications or reoperation entailing implant removal or revision fixation. A quasi-randomised trial comparing arthroscopically-assisted percutaneous reduction and internal fixation versus standard ORIF reported results at 14 months in 58 people with closed Schatzker types II or III tibial plateau fracture. Quality of life, pain and return to pre-injury activity were not reported. There was very low quality evidence of higher HSS knee scores and higher knee range of motion values in the arthroscopically assisted group. No reoperations were reported.

Three trials compared different types of bone substitute versus autologous bone graft (autograft) for managing bone defects. Quality of life, pain and return to pre-injury activity were not reported. Only one trial (25 participants) reported on lower limb function, finding good or excellent results in both groups for walking, climbing stairs, squatting and jumping at 12 months. The incidences of individual complications were similar between groups in all three trials. One trial found no cases of inflammatory response in the 20 participants receiving bone substitute, and two found no complications associated with the donor site in the autograft group (58 participants). However, all 38 participants in the autologous iliac bone graft group of one trial reported prolonged pain from the harvest site. Two trials reported similar range of motion results in the two groups, whereas the third trial favoured the bone substitute group.

\section{Authors' conclusions}

Currently, there is insufficient evidence to ascertain the best method of fixation or the best method of addressing bone defects during surgery. However, the evidence does not contradict approaches aiming to limit soft-tissue dissection and damage or to avoid autograft donor site complications through using bone substitutes. Further well-designed, larger randomised trials are warranted.

\section{PLAIN LANGUAGE SUMMARY}

\section{Surgical fixation methods for treating tibial plateau fractures (breaks in the top end of the shin bone) in adults What is the medical problem?}

Fractures of the tibial plateau are injuries affecting the top end of the tibia (shin bone), which forms the lower bone surface in the knee joint. These fractures are often associated with a large amount of damage to the skin and muscle and may cause voids or defects in the bone.

\section{What treatments are available?}

Often, complex fractures are set surgically through open reduction and internal fixation (ORIF), which entails exposing the fracture to direct view and then fastening the bone fragments into place with metal plates and screws. Another method is external fixation, where wires and pins are placed into the bone around the fracture site to secure the fragments in place until they heal. External fixation can be coupled with the use of small plates or screws to hold some of the fracture fragments in position; this is called hybrid fixation.

To correct possible bone defects, bone void fillers, either bone grafts taken from the patients themselves or bone substitutes, may be used.

Surgical fixation methods for tibial plateau fractures (Review)

Copyright () 2015 The Cochrane Collaboration. Published by John Wiley \& Sons, Ltd. 


\section{What evidence is available?}

We found six small studies (involving 429 adults) of different fixation methods and bone fillers in September 2014. All six trials were small and at substantial risk of bias. We judged the quality of most of the available evidence to be very low, meaning that we are very uncertain about these results

Three studies evaluated different methods of fixation. One study found that hybrid fixation is more likely to result in better quality of life and lower-limb function, fewer complications requiring repeat surgery, and more people returning to pre-injury activity levels than standard ORIF. However, the possibility of a better result from ORIF could not be ruled out. Another study compared a minimally invasive, single-plate technique with a traditional open technique using two plates. This study found very little difference between the two groups in knee function, complications or reoperations. The third study compared arthroscopic surgery (which uses a tiny camera to visualise the joint) and internal fixation versus ORIF. It reported better functional outcome and knee mobility in the arthroscopy group. There were no reoperations.

Three studies compared different bone substitutes versus bone grafts for managing bone defects, but reported on only a few outcomes. One study found similar results in the two groups in the numbers of participants with good walking, stair climbing, squatting and jumping ability at one year. All three studies found similar numbers of specific complications in the two groups. One study found that all participants in the bone graft group had prolonged pain from the harvest site of the bone graft. Two studies reported similar range of motion results in the two groups, whereas the third study found better results in the bone substitute group at one year.

\section{Conclusions}

Currently, there is insufficient evidence to ascertain the best surgical methods of fixation and bone defect treatment for tibial plateau fractures in adults. Well-conducted trials are still needed to inform clinical decision-making.

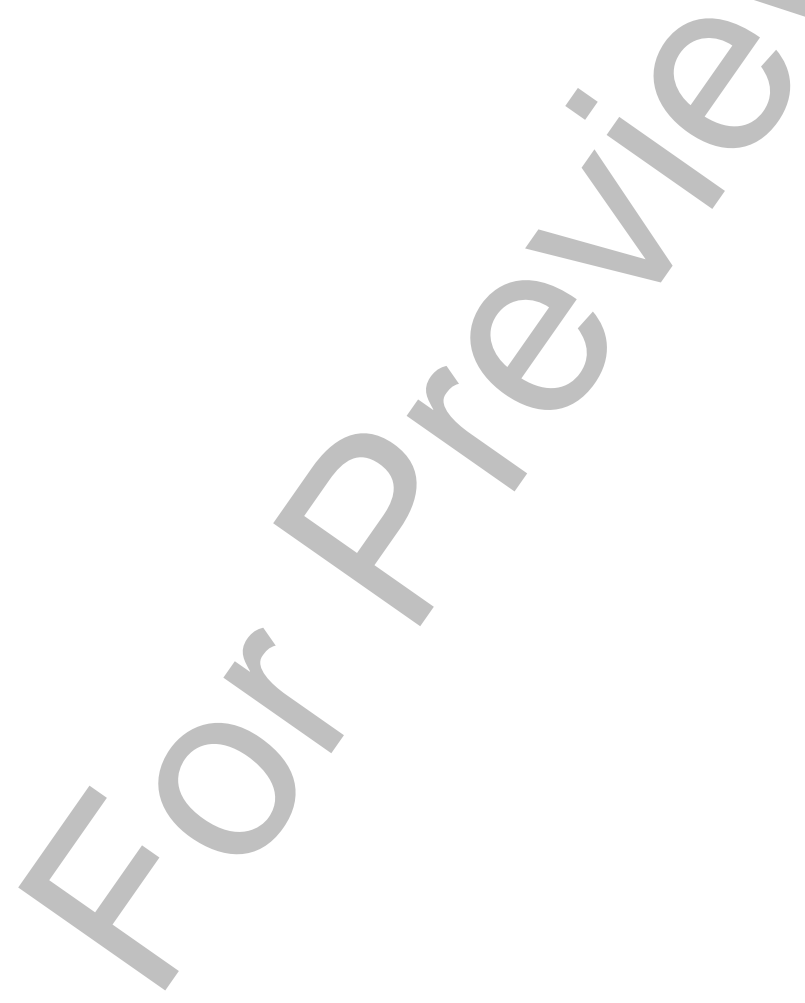

Surgical fixation methods for tibial plateau fractures (Review) 


\begin{tabular}{|c|c|c|c|c|c|c|}
\hline \multicolumn{7}{|c|}{ Hybrid fixation compared with standard open reduction internal fixation for tibial plateau fracture } \\
\hline \multicolumn{7}{|c|}{$\begin{array}{l}\text { Patient or population: adults with tibial plateau fracture (Schatzker types V or VI) } \\
\text { Settings: operating theatres, at } 5 \text { university-affiliated level-I trauma centres in Canada } \\
\text { Intervention: circular external fixator with limited internal fixation (hybrid fixation) } \\
\text { Comparison: standard open reduction internal fixation (plates - no locking plates used) }\end{array}$} \\
\hline \multirow[t]{3}{*}{ Outcomes } & \multicolumn{2}{|c|}{ Illustrative comparative risks* $(95 \% \mathrm{Cl})$} & \multirow[t]{3}{*}{$\begin{array}{l}\text { Relative effect } \\
(95 \% \text { CI) }\end{array}$} & \multirow[t]{3}{*}{$\begin{array}{l}\text { No. of Participants } \\
\text { (studies) }\end{array}$} & \multirow[t]{3}{*}{$\begin{array}{l}\text { Quality of the evidence } \\
\text { (GRADE) }\end{array}$} & \multirow[t]{3}{*}{ Comments } \\
\hline & Assumed risk & Corresponding risk & & & & \\
\hline & Standard ORIF & Hybrid Fixation & & & & \\
\hline 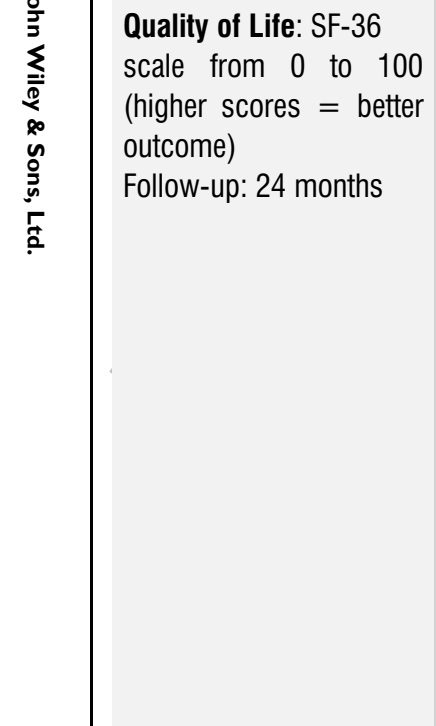 & $\begin{array}{l}\text { See comments } \\
\text { Overall SF-36 score not } \\
\text { reported. }\end{array}$ & See comments & See comments & $66(1)$ & $\begin{array}{l}\oplus \circ \bigcirc \bigcirc \\
\text { very low }{ }^{a}\end{array}$ & $\begin{array}{l}\text { Individual scores were } \\
\text { presented for } 8 \text { domains } \\
\text { of the SF-36 (general } \\
\text { health, physical function, } \\
\text { role-physical, role-emo- } \\
\text { tional, social function, } \\
\text { bodily pain, vitality, men- } \\
\text { tal health). The results } \\
\text { for } 3 \text { domains (physical } \\
\text { function, role-emotional, } \\
\text { bodily pain) all favoured } \\
\text { the hybrid fixation group, } \\
\text { but only the result for } \\
\text { the bodily pain domain } \\
\text { reached statistical signif- } \\
\text { icance (mean } 46 \text { versus } \\
35, \mathrm{MD} 11.00,95 \% \mathrm{Cl} 0 \text {. } \\
66 \text { to } 21.34 ; \mathrm{P}=0.04 \text { ) }\end{array}$ \\
\hline
\end{tabular}




\section{HSS score: \\ The mean HSS score in Mean HSS score in the -}

Scale from 0 to 100

(higher scores indicating point

greater function)

Follow-up: 24 months

xation group was

7.0 points higher (2.4

lower to 16.4 higher)
$66(1)$

$\oplus \oplus \bigcirc \bigcirc$

low $^{b}$

We have not located any reported values for minimal clinically important difference values for the HSS knee score. It is notable that the difference in HSS scores in favour of hybrid fixation was greater at 6 months: mean 72 versus $61, \mathrm{MD}$ 11.00 favouring hybrid fixation, $95 \% 1.64$ to 20 . $36 ; P=0.02)$

\section{WOMAC function The mean WOMAC func- The mean WOMAC func-}

Scale from 0 to $100^{c}$ tion score in the ORIF tion score in the hybrid

(higher scores indicating group was 46.7 points fixation group was 2.4

greater function)

points higher (16.2 lower
pation group was 2.4

Follow-up: 24 months

to 21.0 higher)

Adverse events ${ }^{e}$

Follow-up: 24 months
450 per 1000 people hav- 351 (253 to 594 ) per RR 0.78 ( 0.45 to 1.32) ing a reoperation $f$
1000 people having a re-

operation
83 (1)

( 1 hybrid fixation partici- very lows pant had a bilateral fracture)
$66(1)$

\section{$\oplus \bigcirc \bigcirc \bigcirc$}

very low ${ }^{d}$

Results of the two groups were also comparable for the WOMAC function subscale at 6 and 12 months

99 fewer people per 1000 had an unplanned reoperation after hybrid fixation (95\% Cl 197 fewer to 144 more)

15 out of 43 participants in the hybrid fixation group had 16 unplanned reoperations, compared with 18 participants of 40 in the ORIF group having 37 unplanned reoperations in total over the 2year follow-up period ${ }^{h}$ Reoperations varied from relatively minor proce- 
dures (e.g. screw removal), which tended to occur in the hybrid fixation group, to major procedures (e.g. total knee arthroplasty), which occurred more often in the ORIF group. The authors attributed the markedly shorter length of hospital stay in the hybrid fixation group (mean 9.9 versus 23.4 days; MD -13 . 50 days, $95 \% \mathrm{Cl}-14.77$ to -12.23 days) to participants in the ORIF group requiring multiple procedures for complications

WOMAC Pain The mean WOMAC Pain The mean WOMAC pain subscale from 0 to $100^{c}$ score in the control group score in the intervention (higher scores indicating was 13.1 points

greater function)

Follow-up: 24 months

Knee total range of mo-

tion (degrees)

Follow-up: 24 months

The mean knee range of The mean knee range of motion in the ORIF group motion in the hybrid fixawas 109.0 degrees

tion group was 11.0 degrees higher ( 2.0 degrees lower to 24.0 degrees higher)

Return to pre-injury ac- 121 per $1000^{f}$

tivity level

Follow-up: 24 months

303 (106 to 869$)$ per $\operatorname{RR} 2.50$ (0.87 to 7.18$) \quad 66(1)$

1000

groups was 0.3 points higher (5.9 lower to 5.3

higher)
$66(1)$

$66(1)$

(1)

.

6 (1)

(2)

$\oplus \bigcirc \bigcirc \bigcirc$
very low

Results of the two groups were also comparable for the WOMAC pain at 6 and 12 months

Results of the two groups were also comparable for the WOMAC stiffness subscale at 6, 12 and 24 months

$\oplus \bigcirc \bigcirc \bigcirc$
very low

Thus 182 more people per 1000 had returned to their pre-injury activity level in the hybrid fixation group $(95 \%$ Cl 15 fewer 
*The basis for the assumed risk (e.g. the median control group risk across studies) is provided in footnotes. The corresponding risk (and its $95 \%$ confidence interval) is based on the assumed risk in the comparison group and the relative effect of the intervention (and its $95 \% \mathrm{Cl}$ ).

CI: confidence interval; HSS: Hospital for Special Surgery; MD: mean difference; ORIF: open reduction and internal fixation; SF-36: 36-item Short Form Health Survey;RR: risk ratio; WOMAC: Western Ontario and McMaster Universities Osteoarthritis Index

GRADE Working Group grades of evidence

High quality: Further research is very unlikely to change our confidence in the estimate of effect.

Moderate quality: Further research is likely to have an important impact on our confidence in the estimate of effect and may change the estimate.

Low quality: Further research is very likely to have an important impact on our confidence in the estimate of effect and is likely to change the estimate.

Very low quality: We are very uncertain about the estimate.

${ }^{a}$ The evidence was downgraded one level for study limitations (mainly reflecting a high risk of performance and detection bias relating to

lack of blinding), one level for imprecision (small trial) and one level for indirectness (inadequate outcome measure due to presentation

in 8 domains).

${ }^{b}$ The evidence was downgraded one level for study limitations (mainly reflecting a high risk of performance and detection bias relating to

lack of blinding), and one level for imprecision (small trial).

${ }^{c}$ The scale for this outcome measures was not stated in the trial report, and is thus an assumption based on comments in the text.

${ }^{d}$ The evidence was downgraded one level for study limitations (mainly reflecting a high risk of performance and detection bias relating to lack of blinding), one level for imprecision (small trial), and one level for indirectness (inadequate outcome measure due to underreporting of scale).

${ }^{e}$ Adverse events were complications that resulted in surgery.

${ }^{f}$ Estimate based on trial data.

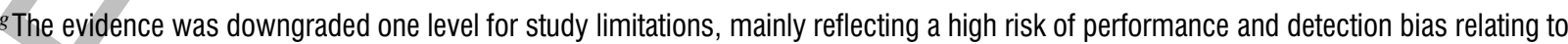
lack of blinding, and two levels for imprecision (wide confidence interval and small trial).

${ }^{h}$ Length of hospital stay was 14 days shorter in the hybrid fixation group (mean 9.9 versus 23.4 days); much of the longer stay in the

ORIF group was attributed to participants requiring multiple procedures for complications in this group.

${ }^{i}$ The evidence was downgraded two levels for study limitations (reflecting a high risk of performance and detection bias relating to lack

of blinding and some inconsistencies in reporting) and one level for imprecision (small trial).

${ }^{j}$ The evidence was downgraded one level for study limitations (mainly reflecting a high risk of performance and detection bias relating

to lack of blinding) and two levels for imprecision (wide confidence interval and small trial). 


\section{B A C K G R O U N D}

\section{Description of the condition}

The knee is the articulating joint between the distal femur (lower end of the thigh bone), the proximal tibia (upper end of the shin bone) and the patella (knee cap). The tibial plateau, which is at the top end of the tibia, forms the lower articulating surface of the knee joint. Anatomically, it comprises the medial (inner half) and lateral (outer half) articulating surfaces; these join with the medial and lateral condyles of the femur, respectively. The medial and lateral portions of the tibial plateau are separated by the tibial spines, between which the anterior cruciate ligament originates. Around $1 \%$ of fractures in adults involve the tibial plateau (Moore 1987). In men, these fractures usually occur at a younger age and often result from high-energy trauma, such as motor vehicle accidents. In women, the fractures occur more often later in life as a result of lower-energy trauma, often reflecting underlying osteoporosis (Schatzker 1979). Reporting on a population of 752 patients with tibial plateau fractures, Moore 1987 found that the average patient age was 44 years and that $62 \%$ of patients were male.

Tibial plateau fractures are always intra-articular fractures. The main classification of tibial plateau fractures is based on the anatomical location of the fracture and the degree of extension of the fracture into the tibial metaphysis (Schatzker 1979). The Schatzker classification establishes six different types of fractures: type I are split fractures of the lateral tibial plateau; type II (the most common) are split-depression fractures of the lateral tibial plateau; type III involve a depression of the lateral tibial plateau; type IV are fractures of the medial plateau; type $\mathrm{V}$ are bicondylar fractures with an intact metaphysis and diaphysis; and type VI are bicondylar fractures in which the shaft is separated from the condyles (Schatzker 1979).

The other widely reported classification was created by the Arbeitsgemeinschaft für Osteosynthesefragen (German for 'Association for the Study of Internal Fixation' or AO) and the Orthopaedic Trauma Association (OTA). The AO/OTA system categorises fractures of the proximal tibia into three main types $(\mathrm{A}, \mathrm{B}, \mathrm{C})$. These in turn are further divided into three groups, each with three subgroups. As intra-articular fractures, fractures of the tibial plateau comprise types B and C (OTA 1996).

Tibial plateau fractures, especially Schatzker types IV to VI, have a high complication rate. Complications include delayed or nonunion of the fracture and early post-traumatic osteoarthritis (Young 1994). Inadequate management of the fracture can result in prolonged disability and pain (Barei 2006; Mallik 1992; Young 1994).

\section{Description of the intervention}

Fractures of the tibial plateau can be treated in a variety of ways. The choice of treatment is influenced by various factors including the type of fracture, associated injuries, bone quality, surgeon preference and experience, and patient expectations and lifestyle (Court-Brown 1990; Laflamme 2003). Typically, health professionals perform a thorough clinical and radiological assessment, including an assessment for associated soft-tissue injuries such as to the menisci and ligaments, which are commonly damaged (Bennett 1994; Shepherd 2002). Simple fractures, such as those with minimal displacement, are usually managed non-operatively with a good outcome (Howard 1997). At the other end of the injury spectrum, significant bone comminution and soft-tissue injury can lead to limb-threatening complications (Young 1994). The management of these injuries often present a significant challenge to the trauma surgeon.

Complex tibial plateau fractures typically require surgical fixation to achieve the best possible functional outcome. Surgical intervention for tibial plateau fractures typically involves open reduction, where the fracture fragments are reduced (put back into place) under direct view, and internal fixation, which uses devices (typically plates and screws) to secure the fractured parts (Egol 2004a). Arthroscopically assisted percutaneous (through the skin) internal fixation has been used for simpler fractures (Schatzker types II and III) (Kayali 2008). Another method used for relatively complex fractures (Schatzker types V and VI) is hybrid fixation, which combines external fixation with limited internal fixation (COTS 2006; Piper 2005). For all three categories, bony voids may be filled with bone graft (either allograft or autograft) or bone graft substitutes.

\section{How the intervention might work}

Surgical treatment aims to achieve anatomical reduction and stability of the articular surface, with the restoration of the length and alignment of the leg (Dirschl 1997; Watson 2002). The aim of surgery is to prevent abnormal loading of the knee joint and secondary degenerative osteoarthritis. During surgery it is important to limit the damage to the surrounding soft tissues in order to preserve the blood supply and vascularity of the fracture fragments, optimise fracture healing and lower the risk of complications (Kumar 2000; Moore 1987).

The standard approach of tibial plateau fracture fixation is the use of a direct technique of open reduction and internal fixation (ORIF), with a variety of implants to reduce and stabilise the fracture. For simpler fractures, such as Schatzker types II and III, using arthroscopically assisted percutaneous reduction and internal fixation methods limits the extent of surgical dissection, but it is a more technically complicated procedure. For complex fractures, the contrasting technique of hybrid fixation may be considered. This is a technique whereby an external fixator is used to apply force to the ligaments and soft tissues of the leg (ligamentotaxis), to reduce the fracture and maintain the reduced position. Various

Surgical fixation methods for tibial plateau fractures (Review) 
other direct techniques, involving limited open incision for placing plates and screws or percutaneously inserted screws, are then used to restore and stabilise the articular surface (Watson 2002). These different procedures have different complications. For instance, for ORIF, a common reason to return to theatre is to remove symptomatic metalwork, while for hybrid fixation, return to theatre is often necessary to adjust the external fixator (COTS 2006; Schatzker 1979).

\section{Why it is important to do this review}

Tibial plateau fractures are often difficult and demanding fractures to treat, with a high risk of complications, including for earlyonset osteoarthritis. Since these fractures occur predominantly in the working population, this injury can have an economic and societal impact. There are variations in current practice in the management of these fractures, one key area being the adoption of hybrid fixation versus open reduction and internal fixation for the most complex types of fractures. These circumstances justify assessment of the evidence surrounding the use of different surgical techniques. It is also necessary to appraise the most recently developed low-profile locking plates and the use of arthroscopically assisted percutaneous fixation methods. Finally, it is unclear whether the type of bone void filler influences the outcome of the fracture fixation. The aim of this review is to inform decisionmaking regarding surgical fixation methods and the type of bone void filler used for tibial plateau fractures.

\section{O B J E C T I VES}

To assess the effects (benefits and harms) of different surgical interventions, and the use of bone void fillers, for treating tibial plateau fractures.

Our intended comparisons included:

- circular external fixation with limited internal fixation (hybrid fixation) versus ORIF;

- different types of internal fixation;

- arthroscopically assisted percutaneous reduction and internal fixation yersus standard ORIF;

- different types of void filler used for bony defects.

\section{METHODS}

\section{Criteria for considering studies for this review}

\section{Types of studies}

We included randomised controlled clinical trials (RCTs) as well as quasi-randomised (method of allocating participants to a treatment which is not strictly random, e.g. by hospital number) trials that compared surgical interventions for treating tibial plateau fractures.

\section{Types of participants}

We included trials involving people undergoing surgical fixation for a tibial plateau fracture.

We excluded trials that principally recruited people following periprosthetic and pathological fractures, as well as trials that principally recruited people with multiple fractures of the same limb. However, we would have included trials that recruited some people in any of the above three categories if separate data had been available or the numbers in these categories were proportionally low (nominally less than 10\%) and balanced between the allocated groups.

We included trials that recruited adults and children unless they were conducted specifically to examine the treatment of paediatric tibial eminence fractures.

\section{Types of interventions}

We included trials comparing different methods of surgical fixation of tibial plateau fractures, such as experimental or novel methods compared with more traditional methods. Potential comparisons of interest included:

- hybrid fixation (as described in 'Description of the intervention') versus ORIF;

- different methods of internal fixation such as for plating;

- arthroscopically assisted percutaneous internal fixation versus standard ORIF.

We also included trials comparing different types of filler for managing bony defects caused by tibial plateau fractures, including bone graft substitutes and autologous (autogenous) bone grafting.

\section{Types of outcome measures}

The primary focus was on long-term functional outcome, measured for at least one year following injury. Where trials reported outcomes longer than one year, we reported these for the full length of the trial as a secondary analysis.

\section{Primary outcomes}

- Generic quality of life measures (for example, the 36-item Short Form Health Survey (SF-36); Ware 1992), with specific reference, where relevant, to the physical and bodily pain components of the score

- Specific, validated patient-reported outcome measures of lower limb function (for example, Hospital for Special Surgery 
(HSS) knee score, Oxford Knee Score (Dawson 1998), Knee Society Clinical Rating score (Insall 1989), Western Ontario and McMaster Universities Osteoarthritis Index (WOMAC), Lower Extremity Functional Scale (LEFS) (Binkley 1999)). We are unaware of any scoring system that has been specifically designed to evaluate the follow-up of patients after knee fractures.

- Serious adverse events (e.g. death, deep infection, septic arthritis, avascular necrosis, complex regional pain syndrome, reoperation, reoperation for removal of metalwork)

\author{
Secondary outcomes \\ - Pain \\ - Intraoperative results (length of surgery, blood loss, length \\ of incision) \\ - Knee range of movement \\ - Return to pre-injury activity level \\ - Hospital length of stay and time off work \\ - Residual deformity and malunion (including the presence \\ of degenerative osteoarthritis) \\ - Patient satisfaction with treatment \\ - Time to radiological union \\ - Total length of treatment
}

\section{Search methods for identification of studies}

\section{Electronic searches}

We searched the Cochrane Bone, Joint and Muscle Trauma Group Specialised Register (12 September 2014), the Cochrane Central Register of Controlled Trials (CENTRAL) (in The Cochrane Library; 2014 Issue 8), MEDLINE (1946 to September Week 1 2014), Ovid MEDLINE In-Process \& Other Non-Indexed Citations (12 September 2014) and EMBASE (1974 to 2014 Week 36). We reviewed unpublished or grey literature using Open Grey (System for Information on Grey Literature in Europe) (4 July 2014). We did not place any constraints on the searches based on language or publication status.

In MEDLINE, we combined a subject-specific search with the Cochrane Highly Sensitive Search Strategy for identifying randomised trials in MEDLINE (sensitivity-maximizing version) (Lefebvre 2011). This strategy was modified for use in the other databases. The search strategies for CENTRAL, MEDLINE and EMBASE are shown in Appendix 1.

We searched for ongoing or recently completed trials in the WHO International Clinical Trials Registry Platform, Current Controlled Trials and clinicaltrials.gov (4 July 2014) (Appendix $1)$.

\section{Searching other resources}

We searched for conference proceedings using the Bone and Joint Journal's Orthopaedic Proceedings search platform (4 July 2014) (Appendix 1). We searched bibliographies of relevant articles identified from the search strategy. In addition, we contacted the corresponding authors from all included studies and trial investigators from ongoing trials to verify the search results.

\section{Data collection and analysis}

The intended methods for data collection and analysis were described in our published protocol (McNamara 2012), which was based on methods described in the Cochrane Handbook of Systematic Reviews of Interventions (Higgins 2011a).

\section{Selection of studies}

Two review authors (IRM and TOS) selected potentially eligible articles by reviewing the title and abstract of each citation. After obtaining full articles, the same review authors independently performed the study selection. In case of disagreement, we reached a consensus through discussion.

\section{Data extraction and management}

Two review authors (IRM and TOS) extracted the data from each study to a pre-defined data extraction form. The data extraction included details related to participant numbers, gender, age and injury, surgical procedure and postoperative rehabilitation, and results related to the relevant outcome measurements, follow-up period and participant drop-out during follow-up. Each review author independently extracted all data before combining it in an agreed data extraction table. We reached a consensus opinion on the results of the data extraction through discussion.

\section{Assessment of risk of bias in included studies}

Two review authors (IRM and TOS) independently assessed the risk of bias of the included studies using Cochrane's 'Risk of bias' tool (Higgins 2011b). We assessed the risk of bias for randomisation (sequence generation and allocation concealment), blinding (including participants, personnel and outcome assessors), completeness of outcome data and selection of reported outcomes. We also evaluated other sources of bias, including bias resulting from major imbalances in key baseline characteristics (e.g. age, gender, fracture type, open fracture, timing of surgery) and performance bias, including the risk of bias from systematic differences in the care provided (e.g. surgeon's experience or rehabilitation).

The risk of bias was categorised as 'low', 'unclear' or 'high' for each of the included studies. When the two authors could not resolve differences between the ratings through discussion, a third author (SD) acted as adjudicator. 


\section{Measures of treatment effect}

We presented risk ratios (RR) for binary data and mean differences (MD) for continuous data. Where different scales or tools were used to measure the same continuous outcome, we planned to calculate standardised mean differences. We presented $95 \%$ confidence intervals (CI) whenever possible.

\section{Unit of analysis issues}

We anticipated that the unit of randomisation for trials in this area would be the individual participant. This hypothesis was confirmed on reviewing the literature. Exceptionally, where a trial included participants with bilateral tibial plateau fractures, data pertained to knees rather than individual participants; this was the case for COTS 2006. In the face of this problem with the unit of analysis, and when we could not make appropriate corrections, we planned to present the data only if the disparity between the unit of analysis and randomisation was small. Where data were pooled, we planned to perform a sensitivity analysis to examine the effect of pooling incorrectly analysed trials with the other correctly analysed trials. However, since only very limited meta-analysis was possible, this analysis was not necessary.

\section{Dealing with missing data}

We contacted all corresponding authors for key information that was missing from their respective publications. Where appropriate, we planned to perform an intention-to-treat analysis, designed to include all participants randomised to the intervention groups. We planned to assess the effect of any participants who dropped out or who were excluded from the trials by conducting worstand best-case scenario analyses. We were alert to the potential mislabelling or non-identification of standard errors and standard deviations. Unless missing standard deviations could be derived from confidence intervals or exact $\mathrm{P}$ values, we did not assume values for presentation in the analysis.

\section{Assessment of heterogeneity}

In making decisions on pooling, we assessed the clinical heterogeneity of participants, interventions and outcomes of the included studies. Where pooling was done, we assessed statistical heterogeneity by visually inspecting forest plots and by using the $\mathrm{I}^{2}$ and $\mathrm{Chi}^{2}$ statistical tests. We intended to interpret $\mathrm{I}^{2}$ values according to Deeks 2011, which states that an $\mathrm{I}^{2}$ value of $0 \%$ to $40 \%$ might 'not be important'; $30 \%$ to $60 \%$ may represent 'moderate' heterogeneity; $50 \%$ to $90 \%$ may represent 'substantial' heterogeneity; and $75 \%$ to $100 \%$ represents 'considerable' heterogeneity.

\section{Assessment of reporting biases}

Had 10 or more studies met the inclusion criteria for a metaanalysis, we would have used funnel plots to assess publication bias (Sterne 2011).

\section{Data synthesis}

Initially, we planned to pool the results of comparable groups of trials using a fixed-effect model. If the data demonstrated statistically significant heterogeneity, especially when this was unexplained, we planned to use a random-effects model. Where there was excessive heterogeneity, we planned to present the data narratively in the text. However, only very limited pooling for one outcome measure was performed.

\section{Subgroup analysis and investigation of heterogeneity}

If there is sufficient data in a future update, we intend to undertake the following subgroup analyses to examine potential differences in treatment effect.

- Different fracture types as categorised using the Schatzker classification (types I to VI).

- Open versus closed fractures.

- Timing of surgery (i.e. immediate versus delayed).

- Use versus non-use of bone grafting to fill bone defects at the time of initial surgery.

We will also investigate whether the results of subgroups are significantly different by inspecting the overlap of CIs and performing the test for subgroup differences available in Review Manager (RevMan).

\section{Sensitivity analysis}

When appropriate, we planned to conduct sensitivity analyses to examine the various aspects of trial and review methodology, including inclusion of trials at high risk of bias (primarily a lack of allocation concealment). However, due to the limitations of the evidence base, we did not undertake these analyses.

\section{'Summary of findings' tables}

We used the GRADE approach to assess the quality of evidence related to each of the listed primary outcomes and the secondary outcome measures of pain, knee range of movement and return to pre-injury activity level (Types of outcome measures) (Higgins 2011a). We presented the results for one comparison in a 'Summary of findings' table.

\section{RE S U L T S}




\section{Description of studies}

See: Characteristics of included studies; Characteristics of excluded studies; Characteristics of ongoing studies.

\section{Results of the search}

We completed the search in September 2014. We screened a total of 1093 records from the following databases: Cochrane Bone, Joint and Muscle Trauma Group Specialised Register (10 records); CENTRAL (33), MEDLINE (206), EMBASE (235), the WHO International Clinical Trials Registry Platform (14), Current Controlled Trials (276), ClinicalTrials.gov (19), the Journal of Bone and Joint Surgery's Orthopaedic Proceedings (199) and Open Grey (101). We also screened 194 additional records identified through other sources.

Our search identified 33 potentially eligible papers (29 studies), for which we obtained full reports. Six trials were included in total: three evaluated different surgical techniques (COTS 2006; Jaing 2008; Shen 2011), and three evaluated different types of bone graft filler (Bucholz 1989; Heikkilä 2011; Russell 2008).

We excluded 19 trials (Carter 1996; Chan 2012; Dall'oca 2012; Egol 2004b; Gunther 2008; Hansen 2009; Jubel 2004; Krupp 2009; Lobenhoffer 1999; Malakasi 2013; NCT00429585; NCT00610701; Ohdera 2003; Ong 2012; Sung 2008; Tahririan 2014; Weaver 2012; Zhang 2012; Zhuo 2008).

We identified three studies (five articles) through the clinical trials registries as ongoing and awaiting publication (Kuros Biosurgery AG; NCT01828905; NCT02035917). Another study of unknown status (awaiting classification) is investigating the use of balloon osteoplasty for fracture reduction (Jordan 2014).

The flow diagram of the search strategy results is presented in Figure 1. 
Figure I. Study flow diagram.

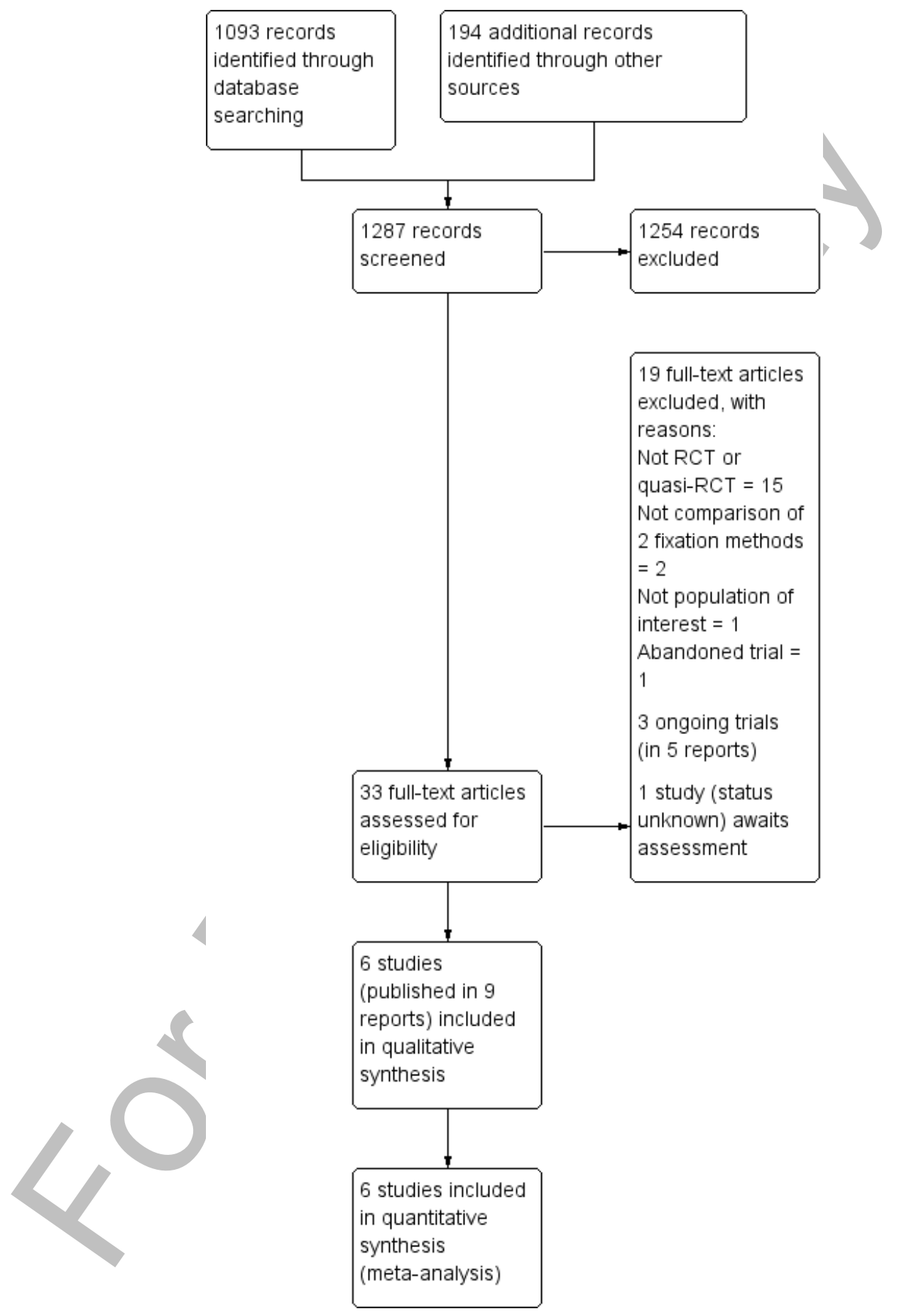




\section{Included studies}

The six included trials were published between 1989 and 2011. There were three reports for COTS 2006 and two for Heikkilä 2011. Five were published in English (Bucholz 1989; COTS 2006; Heikkilä 2011; Jaing 2008; Russell 2008) and one was translated from Chinese (Shen 2011).

For more details of individual studies, please see the Characteristics of included studies table.

\section{Design}

Four trials were randomised controlled trials (COTS 2006; Heikkilä 2011; Jaing 2008; Russell 2008), and two were quasirandomised controlled trials (Bucholz 1989; Shen 2011). All six were parallel, two-group trials.

Participants were probably allocated in a 1:1 ratio in four trials; however, there was a notable imbalance in allocation in Shen 2011, where 38 patients were allocated to one group and 20 to the other group. Russell 2008 based randomisation into groups for autologous iliac bone graft or alpha-BSM on a purposefully 1 : 2 protocol because autologous iliac bone graft was considered to be associated with higher risks of morbidity to the patient.

\section{Sample sizes}

Sample sizes in the included studies range from 25 (Heikkilä 2011) to 119 (Russell 2008) randomised participants, with 15 (Heikkilä 2011) to 94 (Russell 2008) participants in the final analyses.

\section{Settings}

One trial was performed in Canada (COTS 2006), two in China (Jaing 2008; Shen 2011), one in Finland (Heikkilä 2011) and two in the USA (Bucholz 1989; Russell 2008). Two were multicentre trials (COTS 2006; Russell 2008), while the rest were singlecentre.

\section{Participants}

Of the six trials, three evaluated different surgical techniques (COTS 2006; Jaing 2008; Shen 2011), and three evaluated different types of bone graft filler (Bucholz 1989; Heikkilä 2011; Russell 2008). Below, we describe patient characteristics according to these intervention types.

\section{Surgical fixation}

COTS 2006 included 82 participants (83 fractures) presenting with an open or closed, displaced, bicondylar tibial plateau fracture (Schatzker types V and VI). The mean age of the study population was 45 years, and $60 \%$ were male.

Jaing 2008 included 96 participants presenting with an open or closed, bicondylar tibial plateau fracture (AO/OTA types $\mathrm{C} 1$ to C3). The study did not report group allocation of 12 exclusions following randomisation. The mean age of the 40 participants in the analyses was 42 years, and $69 \%$ were male.

Shen 2011 included 58 participants presenting with closed, unilateral tibial plateau fractures (Schatzker types II and III). The mean age of the study population was 36 years (range 20 to 56 years), and $69 \%$ were male.

\section{Bony void fillers}

Bucholz 1989 included 49 participants presenting with closed, unstable tibial plateau fractures. Nine participants lost to followup were excluded. The mean age of the 40 participants included in the analyses was 37 years (range 16 to 73 years), and 60\% were male.

Heikkilä 2011 included 25 participants presenting with closed, unilateral tibial plateau fractures (AO classification $41 \mathrm{~B} 2$ and B3). The mean age of the study participants was 54 years (range 25 to 82 years), and $48 \%$ were male.

Russell 2008 included 119 participants presenting with 120 closed, unstable tibial plateau fractures (Schatzker types I to VI). The mean age of the study population was 43 years, and $61 \%$ were male.

\section{Surgical interventions}

\section{Surgical fixation}

Three trials tested one each of the following comparisons of surgical fixation methods.

- Circular external fixation with limited internal fixation (hybrid fixation) versus standard open reduction internal fixation (ORIF) (COTS 2006).

- Less invasive stabilisation system (LISS) versus doubleplating open reduction internal fixation (Jaing 2008).

- Arthroscopically assisted percutaneous reduction and internal fixation versus standard open reduction and internal fixation (Shen 2011). 


\section{Bony void fillers}

Three studies compared the following bone-fillers with autologous bone graft.

- Interporous hydroxyapatite (Bucholz 1989).

- Bioactive glass (Heikkilä 2011).

- Endothermic calcium phosphate cement (Russell 2008).

\section{Outcomes}

\section{Surgical fixation}

Trial participants were followed up for an average of 13 months (between 12 to 14 months) in Shen 2011 and for 24 months in COTS 2006 and Jaing 2008.

Only COTS 2006 presented quality of life data (based on SF36 scores) and WOMAC scores. All three surgical fixation trials reported Hospital for Special Surgery (HSS) knee scores. None of these scoring systems was specifically designed to evaluate the follow-up of patients following trauma.

All three trials reported on complications, but only two trials presented reoperation data (COTS 2006; Jaing 2008).

\section{Bony void fillers}

Follow-up duration was one year in Heikkilä 2011 and Russell 2008. However, Heikkilä 2011 also reported long-term results at 11 years and final radiographs were taken at between 12 and 48 months in Russell 2008. Mean follow-up was 34.5 months in the bone substitute group of Bucholz 1989, compared with 15.4 months in the autograft group.

None of the three trials reported data for quality of life or validated knee scores. Heikkilä 2011 reported on the numbers of participants with excellent or good lower limb function based on ability to walk, climb stairs, squat and jump.

All three studies reported on complications.

\section{Excluded studies}

We excluded 16 of the 19 excluded trials because they were not randomised or quasi-randomised controlled trials (Carter 1996;
Chan 2012; Dall'oca 2012; Egol 2004b; Gunther 2008; Hansen 2009; Jubel 2004; Krupp 2009; Lobenhoffer 1999; Malakasi 2013; Ohdera 2003; Ong 2012; Tahririan 2014; Weaver 2012; Zhang 2012; Zhuo 2008). One study did not compare specific fixation techniques (Sung 2008), one currently ongoing, excluded study focused on patients with extracapsular proximal tibial fractures without intra-articular extension (NCT00429585), and one registered trial was abandoned (NCT00610701).

For further details, please see the Characteristics of excluded studies table.

\section{Ongoing studies}

Three studies are ongoing or awaiting publication (Kuros Biosurgery AG; NCT01828905; NCT02035917).

\section{Surgical fixation}

One potentially randomised trial aims to compare non-locking and locking plate fixation in people with unilateral closed tibial plateau fractures (NCT02035917).

\section{Bone void fillers}

One trial aims to compare CERAMENTTM I BONE VOID FILLER versus autologous cancellous bone graft (iliac crest) in 136 patients with tibial plateau fractures treated by internal fixation and void reconstruction. Another trial, which is an industrysponsored phase 2 randomised trial, aims to compare the safety and efficacy of two doses of a novel bone substitute (I-0401; also KUR-111) with autologous bone graft (Kuros Biosurgery AG). At the time of writing, this trial had completed recruitment of at least 183 patients with tibial plateau fractures.

\section{Risk of bias in included studies}

We present our judgements regarding the risk of bias in each of seven domains for individual studies in Figure 2 and summarise them in Figure 3. 
Figure 2. Risk of bias summary: review authors' judgement about each risk of bias item for each included study.

\begin{tabular}{|c|c|c|c|c|c|c|c|}
\hline & 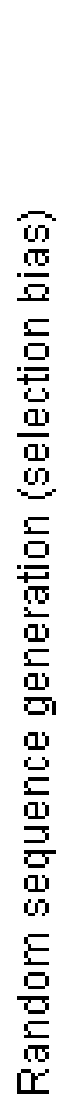 & 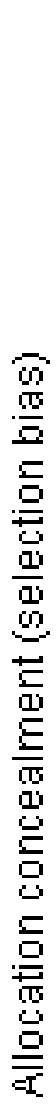 & 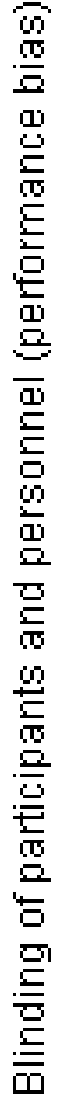 & 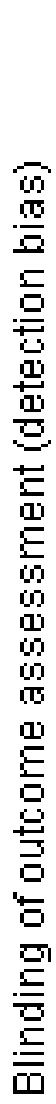 & 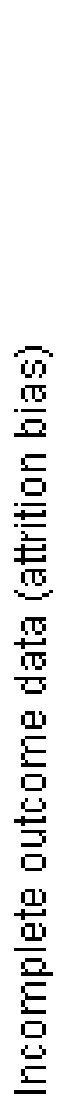 & 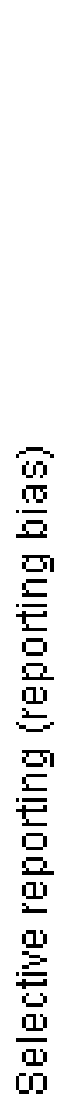 & 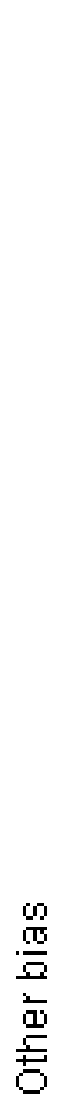 \\
\hline Bucholz 1989 & & & & & & $?$ & $?$ \\
\hline COTS 2006 & & & & & $?$ & $?$ & \\
\hline Heikkilä 2011 & $?$ & $?$ & & & $?$ & & $?$ \\
\hline Jaing 2008 & & $?$ & & & & $?$ & \\
\hline Russell 2008 & & & & & & $?$ & $?$ \\
\hline Shen 2011 & & & & & & $?$ & \\
\hline
\end{tabular}


Figure 3. Risk of bias graph: review authors' judgement about each risk of bias item presented as percentages across all included studies.

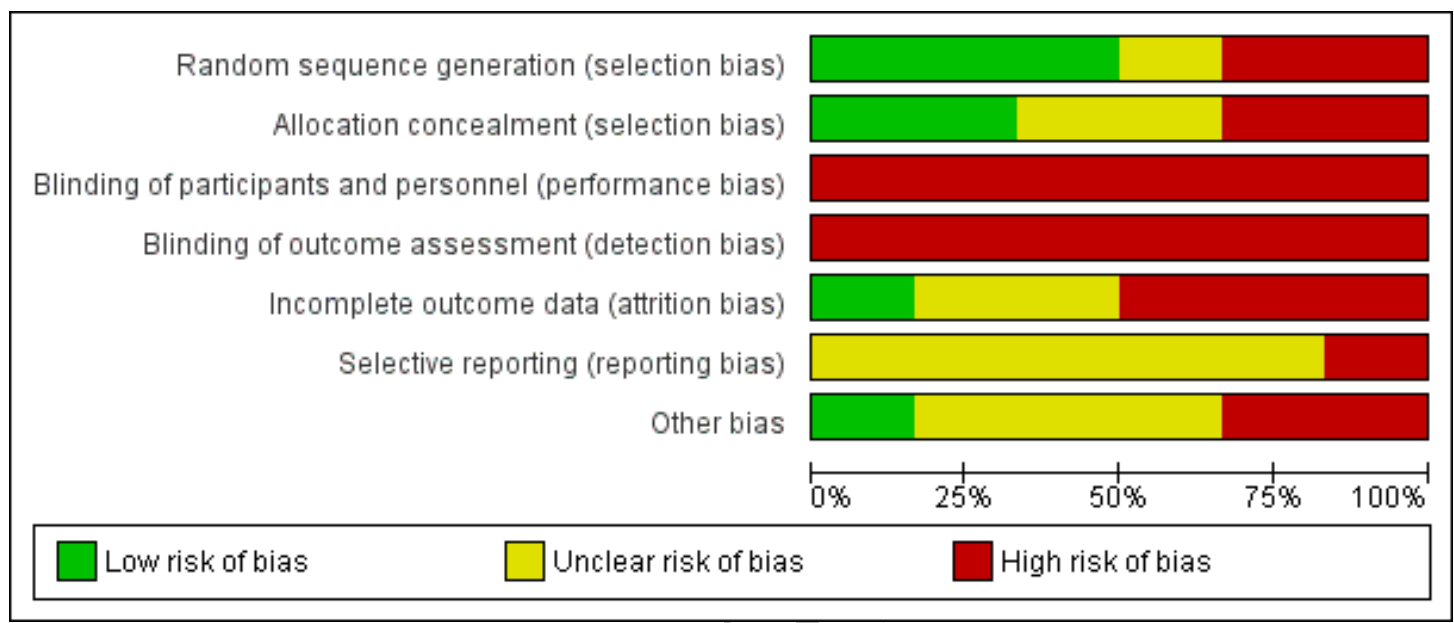

\section{Allocation}

We deemed both multi-centre trials to be at low risk of selection bias, both in terms of sequence generation and allocation concealment (COTS 2006; Russell 2008). We judged both quasi-randomised trials to be at high risk of selection bias, reflecting the inadequacy of the allocation concealment due to the predictability of the allocation sequencing (Bucholz 1989; Shen 2011). Heikkilä 2011 reported the use of closed envelopes but provided no other details on randomisation, so we judged its risk of bias to be unclear for both random sequence generation and allocation concealment. Jaing 2008, which reported only on the use of computer-generated random numbers, carried a low risk of bias related to sequence generation but an unclear risk for allocation concealment.

\section{Blinding}

All six trials were judged at high risk of performance bias due to lack of blinding. The contrast between the interventions being tested meant that blinding of surgeons and participants was not practical. Likewise, all six trials were judged at high risk of detection bias. There was no report of assessor blinding in the trials aside from blinding of panellists assessing radiographs in Jaing 2008. However, even for these outcomes, Jaing 2008 acknowledged that the reviewers may have been able to detect the intervention used, which undermined the blinding.

\section{Incomplete outcome data}

No loss to follow-up occurred in Shen 2011, which was at low risk of attrition bias. COTS 2006 reported similar losses in the two intervention groups, and Heikkilä 2011 may not have declared the total number randomised; we considered that both of these studies had an unclear risk of attrition bias. Notably, the long-term followup data from Heikkilä 2011 were at high risk of attrition bias. Both Bucholz 1989 and Jaing 2008 were at high risk of bias due to their failure to provide group assignments for postrandomisation exclusions or losses to follow-up. Russell 2008 was also at high risk of attrition bias from an imbalance in the losses between the two groups ( $18 \%$ versus $31 \%$ ).

\section{Selective reporting}

All studies reported the outcomes that had been defined in their 'Methods' sections. However, there was no indication in any report of trial registration on whether a trial protocol had been published, nor did we find any. With the exception of Heikkilä 2011, to which we assigned a high risk of bias since numerical data were often unavailable, we judged all the trials to have unclear risk of bias for reporting bias.

\section{Other potential sources of bias}


With regard to the risk of other sources of bias, mainly judged on whether the baseline characteristics between intervention groups were comparable, we judged it to be low for COTS 2006; unclear for Bucholz 1989, Heikkilä 2011 and Russell 2008; and high for Jaing 2008 and Shen 2011. In Jaing 2008, more participants in the double-plating group had primary bone graft, meniscal repair and cruciate ligament surgery; these may point to performance bias, differences in baseline characteristics or both. The unexplained imbalance in the numbers in the two groups and the lack of details and control of fixation methods put Shen 2011 at high risk of other bias.

\section{Effects of interventions}

See: Summary of findings for the main comparison Circular external fixation with limited internal fixation (hybrid fixation) versus standard open reduction internal fixation

\section{Circular external fixation with limited internal fixation (hybrid fixation) versus standard open reduction internal fixation}

COTS 2006 compared circular external fixation with limited internal fixation (hybrid fixation) with ORIF in 82 people who had open or closed Schatzker types V or VI tibial plateau fractures. Results were available for 66 participants at 24 months follow-up.

\section{Primary outcomes}

\section{Generic quality of life measures}

The distribution of the scores at 24 months for the individual domains making up the SF-36 (general health, physical function, role-physical, role-emotional, social function, bodily pain, vitality, mental health) are shown in Analysis 1.1 (all SDs were calculated from reported P values). The results for three domains (physical function, role-emotional, bodily pain) all favoured the hybrid fixation group but only the result for the bodily pain domain reached statistical significance (mean 46 versus 35, MD 11.00, 95\% CI 0.66 to $21.34 ; \mathrm{P}=0.04 ; 66$ participants).

\section{Validated specific patient-reported outcome measures of lower limb function}

Hospital for Special Surgery (HSS) scores were higher in the hybrid fixation group at 6,12 and 24 months, but the difference between the two groups was only statistically significant (and probably included a clinically important difference) at 6 months (mean 72 versus 61, MD 11.00 favouring hybrid fixation, 95\% 1.64 to 20.36; $\mathrm{P}=0.02$; 76 participants; Analysis 1.2). WOMAC scores for the pain, stiffness and function at 6,12 and 24 months were comparable in the two groups (Analysis 1.3; Analysis 1.4; Analysis
1.5). The range of each of these scores was not given in the trial report but is likely to be 0 to 100 , with higher scores indicating worse outcome. In their discussion, COTS 2006 observed that there was higher disability measured by the WOMAC subscale of function (range of participant scores 41 to 56) than in pain (range 11 to 15) or stiffness (range 7 to 10). The greatest between-group difference for the WOMAC subscales was for function at six months (mean 56.1 versus 46.1; MD 10.00 favouring hybrid fixation, $95 \% \mathrm{CI}$ - 6.71 to 26.71; $\mathrm{P}=0.24 ; 76$ participants; Analysis 1.5 ).

\section{Serious adverse events}

Two participants allocated to hybrid fixation underwent ORIF because reduction of their OTA type $\mathrm{C} 3$ fractures was not possible; their results were included in those for hybrid fixation. Slightly fewer participants of the hybrid fixation group underwent unplanned reoperations following initial surgery (15/43 versus $18 /$ 40; RR $0.78,95 \%$ CI 0.45 to 1.32; Analysis 1.6). Of the participants requiring an unplanned reoperation, 15 people in the hybrid fixation group had a total of 16 reoperations in total, whereas the 18 participants in the ORIF group who had a reoperation required 37 reoperations over the two-year follow-up period. The types and proportions of reoperation are shown in Analysis 1.6. Reoperations varied from minor procedures, which occurred more often in the hybrid fixation group (screw removal in 6/43 people compared to $0 / 40$ in the ORIF group), to major interventions, which occurred more often in the ORIF group (total knee arthroplasty (1/43 versus $2 / 40)$, amputation $(0 / 43$ versus $1 / 40)$ ).

\section{Secondary outcomes}

\section{Intra-operative results}

Length of surgery was on average 13 minutes less in the hybrid fixation group (mean 170 versus 183 minutes; MD -13.00 minutes, $95 \%$ CI -34.02 to 8.02 minutes; Analysis 1.7), and blood loss in the hybrid fixation group was under half that of the ORIF group (213 ml versus $544 \mathrm{ml}$; MD $-331.00 \mathrm{ml}$, 95\% CI -560.88 to $-101.12 \mathrm{ml}$; Analysis 1.7$)$. SDs were calculated from reported $P$ values for both outcomes.

\section{Knee range of movement}

The total arc of motion was greater in the hybrid fixation group (mean 120 versus 109 degrees, MD 11.00 degrees, 95\% CI 1.99 to 23.99; Analysis 1.8), this reflected higher flexion range of motion values in this group. 


\section{Return to pre-injury activity level}

Although return to pre-injury activity level by the end of each follow-up was low in both groups, a higher proportion returned in the hybrid fixation group at each stage (Analysis 1.9). At 6 months, the results were $8 / 40$ versus $1 / 36$ (RR $7.20,95 \%$ CI 0.95 to 54.79 ); at 12 months, $10 / 37$ versus $2 / 35$ (RR $4.73,95 \% \mathrm{CI}$ 1.11 to 20.09); and at 24 months, $10 / 33$ versus $4 / 33$ (RR 2.50, $95 \%$ CI 0.87 to 7.18 ), for the hybrid fixation and ORIF groups, respectively.

\section{Hospital length of stay}

Length of hospital stay was significantly shorter in the hybrid fixation group: mean 9.9 versus 23.4 days; MD -13.50 days, $95 \% \mathrm{CI}$ -14.77 to -12.23 days; Analysis 1.10). The trial authors attributed the longer stay in the ORIF group primarily to participants in this group requiring multiple procedures for complications.

\section{Residual deformity and malunion}

Postoperatively, the quality of reduction between the groups was compared radiographically. As illustrated in Analysis 1.11, the results for the various parameters reported were comparable between the two groups. At both one and two years follow-up, similar numbers in the two groups had radiographic signs of osteoarthritis (joint space narrowing, osteophyte formation and sharpening of tibial spines); at 24 months follow-up, results were $13 / 32$ versus $11 / 34$ for the hybrid fixation and ORIF groups, respectively (RR 1.26, $95 \%$ CI 0.66 to 2.39; Analysis 1.12 ).

\section{Other secondary outcomes}

There were no outcomes reporting patient satisfaction with treatment, time to radiological union or total length of treatment.

\section{Less invasive stabilisation system (LISS) versus double-plating open reduction internal fixation}

Jaing 2008 reported results up to 24 months, comparing the less invasive stabilisation system (LISS) with double-plating open reduction internal fixation in 84 people who had open or closed bicondylar tibial plateau fractures (AO/OTA types C1, C2 or C3).

\section{Primary outcomes}

\section{Generic quality of life outcome measure}

No generic quality of life measure was recorded.
Specific, validated patient-reported outcome measures of lower limb function

At 12 and 24 months follow-up, there were no clinically important differences between the two groups (12 months: mean 81.8 versus 80.3; MD 1.50 favouring LISS, 95\% CI -0.94 to 3.94; 24 months: 83.3 versus 83.1 ; MD 0.20, $95 \%$ CI -2.12 to 2.52); see Analysis 2.1 .

\section{Serious adverse events}

Jaing 2008 provided a comprehensive summary of individual complications (Analysis 2.2). None of the between-group differences in individual complications were statistically significant. The most frequent complication was seroma (8/41 in the LISS group versus $5 / 43$ in the double-plating group; RR $1.68,95 \%$ CI 0.60 to 4.71 ), followed by infection, reported as either deep infection (3/41 versus 2/43; RR $1.57,95 \%$ CI 0.28 to 8.94 ) or superficial infection (4/41 versus $3 / 43$; RR $1.40,95 \%$ CI 0.33 to 5.87 ). Of particular note are the five cases of hardware irritation in the LISS group (5/ 41 versus $0 / 43$; RR $11.52,95 \%$ CI 0.66 to 202.02 ) and one case of implant failure in the double-plating group (0/41 versus $1 / 43$; RR $0.35,95 \%$ CI 0.01 to 8.34). Jaing 2008 reported on the total complications (26/41 versus $21 / 43$ ) but this may not equal the total number of participants who experienced complications.

During the 24-month follow-up, seven participants underwent LISS implant removal and four participants underwent doubleplate removal (7/41 versus 4/43; RR 1.84 favouring double-plating, $95 \%$ CI 0.58 to 5.81 ). One case of revision fixation occurred in the double-plating group (0/41 versus 1/43) (Analysis 2.3).

\section{Secondary outcomes}

\section{Intraoperative results}

Length of surgery was on average 16 minutes less in the LISS group (mean 127 versus 143 minutes; MD -16 minutes, 95\% CI -28.02 to -3.98 minutes; Analysis 2.4). The surgical incision length was also smaller in the LISS group (mean 13.3 versus $18.4 \mathrm{~cm}$; MD -5.1 cm, 95\% CI -5.53 to -4.67; Analysis 2.5). Similarly, perioperative blood loss was lower in the LISS group (mean $347 \mathrm{ml}$ versus $410 \mathrm{ml}$; MD -63.0 ml, 95\% CI -100.41 to -25.59; Analysis 2.6). Notably, a greater proportion of patients in the double-plating group received bone graft $(22 / 43 ; 51 \%)$ compared with the LISS group $(12 / 41 ; 29 \%)$. The reasons for the decisions to use bone grafts by the treating surgeons were not reported.

\section{Residual deformity and malunion}

Postsurgical malreduction was reported in two participants of the LISS group versus three participants in the double-plating group (RR 0.70, 95\% CI 0.12 to 3.97; Analysis 2.7). The incidence 
of postsurgical malalignment was higher in the LISS group (6/ 41 versus 1/43; RR 6.29, 95\% CI 0.79 to 50.03; Analysis 2.7). Similar numbers in the two groups subsequently sustained loss of reduction (1/41 versus 2/43) and loss of alignment (2/41 versus 1/43); see Analysis 2.2.

\section{Time to radiological union}

One case of nonunion, defined as no evidence of healing after six months, occurred in the double-plating group (Analysis 2.2); this eventually resolved after treatment with an exchange implant and bone graft. Radiologically assessed healing took place on average one week earlier in the LISS group (mean 14.1 versus 15.3 weeks; MD -1.20 weeks, 95\% CI -1.89 to -0.51 weeks; Analysis 2.8).

\section{Other secondary outcomes}

There was no report on pain, knee range of movement, return to pre-injury activity level, range of movement, length of hospital stay, patient satisfaction with treatment, total length of treatment or incidence of radiologically-detected osteoarthritis.

\section{Arthroscopically assisted percutaneous reduction and internal fixation versus standard open reduction and internal fixation}

Shen 2011 reported the results up to 14 months comparing arthroscopically assisted percutaneous reduction and internal fixation versus standard ORIF in 58 people who had Schatzker types II or III tibial plateau fractures.

\section{Primary outcomes}

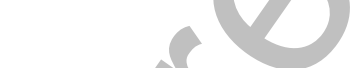

Generic quality of life outcome measure

No generic quality of life measure was reported.

Validated specific patient-reported outcome measures of lower limb function

At 14 months follow-up, HSS knee scores were higher in the arthroscopically assisted group than the ORIF group (mean 86.5 versus 73.4; MD 13.13 favouring arthroscopically assisted surgery; 95\% CI 9.00 to 17.26 ; Analysis 3.1 ).

\section{Serious adverse events}

Shen 2011 reported an absence of serious adverse events, specifically implant loosening or breakage and postoperative fracture. They reported a smaller incidence of knee stiffness in the arthroscopically assisted group (2/38 versus $3 / 20$; RR 0.35 favouring arthroscopically assisted surgery, $95 \% 0.06$ to 1.93 ; Analysis 3.2).
All cases were relieved after an intra-articular injection of sodium hyaluronate. Painkillers eased joint pain in six ORIF group participants, three of whom also had knee stiffness. All wounds healed.

\section{Secondary outcomes}

\section{Pain}

As above, six cases of early and resolved joint pain occurred in the ORIF group.

\section{Intraoperative results}

Arthroscopically assisted percutaneous internal fixation took longer to perform compared with ORIF (length of surgery: mean 107.32 minutes versus 90.5 minutes; MD 16.82 minutes favouring ORIF; 95\% CI 7.05 to 26.59 minutes; Analysis 3.3). Incision length was, as expected, shorter in the arthroscopically assisted surgery group (mean $6.53 \mathrm{~cm}$ versus $14.73 \mathrm{~cm}$; MD -8.2 $\mathrm{cm}$ favouring arthroscopically assisted surgery; $95 \% \mathrm{CI}-8.95$ to $7.45 \mathrm{~cm}$; Analysis 3.4).

\section{Knee range of movement}

At 12 to 14 months follow-up, the arthroscopically assisted percutaneous internal fixation group had a greater knee range compared with the ORIF group (mean 125.45 degrees versus 114.78 degrees; MD 10.67 degrees, 95\% CI 2.74 to 18.60; Analysis 3.5).

\section{Residual deformity and malunion}

Shen 2011 did not report on malalignment or on degenerative osteoarthritis.

\section{Time to radiological union}

All fractures healed in both groups. The mean time to radiological union in the arthroscopically assisted percutaneous internal fixation group was half a month shorter than that in the ORIF group (time to union 3.3 months versus 3.8 months; MD 0.5 months; $95 \%$ CI 0.17 to 0.85 months; Analysis 3.6).

\section{Other secondary outcomes}

There were no outcomes specifically examining return to pre-injury activity levels, blood loss, hospital length of stay, degenerative osteoarthritis, patient satisfaction to treatment or total time of treatment in the different groups. 


\section{Bone substitute versus autologous bone for filling bony voids}

Three trials, testing three different bone substitutes, made this comparison. Only very limited pooling (for individual complications) was possible.

Bucholz 1989 tested interporous hydroxyapatite and reported the results up to 15 months in 40 participants who had closed unstable tibial plateau fractures. Heikkilä 2011 tested bioactive glass and reported the results up to 12 months in 25 participants who had closed unilateral tibial plateau fractures. Heikkilä 2011 also reported long-term follow-up results at 11 years in 15 participants. Russell 2008 tested an endothermic calcium phosphate cement, and reported results at 12 months in 92 participants (93 fractures) who had closed unstable tibial plateau fractures (Schatzker types I to VI). Russell 2008 also reported on radiographic results collected in 102 fractures (101 participants) between 12 and 48 months.

\section{Primary outcomes}

\section{Generic quality of life outcome measure}

None of the three trials reported any generic quality of life measures.

\section{Specific, validated patient-reported outcome measures of lower limb function}

No formal outcome score assessing functional outcome was reported in the main reports of any of the three trials. In the long term follow-up report of Heikkilä 2011, investigators reported that the subjective functional outcome and the median for the knee injury and osteoarthritis outcome score (KOOS) - except for the sport score - were slightly higher in the bioglass group than in the autograft group at 11 years follow-up. However, Heikkilä 2011 provided no data and did not perform any statistical analyses of these outcomes. The earlier report (25 participants) found similar proportions of participants in the two groups with excellent or good lower limb function expressed in terms of ability to walk, climb stairs, squat and jump (Analysis 4.1).

\section{Serious adverse events}

The incidence of individual complications were similar in the bone substitute and autograft groups in all three trials. Only data for infection could be pooled (4/116 versus 3/69 in the bone substitute and autograft groups, respectively; RR $1.01,95 \%$ CI 0.27 to 3.82 , 3 trials; Analysis 4.2). We present accounts of the complications reported in the three trials below.

Bucholz 1989 reported seven complications in the hydroxyapatite group (1 loss of reduction, 3 prominent plates, 1 loose screw and 2 deep infections; the loss in reduction and one of the deep infections occurred in the same patient) and 11 complications in the autograft group ( 3 cases of loss of reduction; 3 cases of prominent plates; 3 loose plates and 2 deep wound infections; it is not clear if any of the participants of this group had more than one complication). The two participants with deep infections in each group had subsequent surgery.

Heikkilä 2011 reported that three participants required blood transfusion (1/14 bioactive glass versus $2 / 11$ autograft) due to excessive bleeding (> $500 \mathrm{ml}$ ), and one participant in the bioactive glass group had a superficial wound infection that was treated with debridement.

Russell 2008 reported one surgical site wound infection in each group (1/82 versus 1/38), both resolved with local wound care and antibiotics. No participants in Russell 2008 had plate breakage or an unplanned reoperation.

Bucholz 1989 reported no cases of inflammatory response to the bone substitute. Neither Bucholz 1989 nor Russell 2008 found complications associated with the donor site in the autograft group. However, all participants in the autologous iliac bone graft group of Russell 2008 had postoperative pain from the harvest site that took 6 to 12 months to resolve.

\section{Secondary outcomes}

\section{Pain}

Pain was not reported fully in any of the three trials. Although Bucholz 1989 noted that "significant knee pain" unrelated to prominent hardware was present in five participants (2/20 hydroxyapatite group versus $3 / 20$ bone graft group), this observation does not constitute a complete report of knee pain.

\section{Intraoperative results}

Neither Bucholz 1989 nor Russell 2008 recorded intraoperative outcomes. The mean drain bleeding from the operation area of the tibia reported by Heikkilä 2011 was $195 \mathrm{ml}$ (0 to $1000 \mathrm{ml})$ in the bioglass group versus $180 \mathrm{ml}(0$ to $1390 \mathrm{ml})$ in the autograft group. Additionally, the mean bleeding from iliac crest was 215 $\mathrm{ml}(0$ to $960 \mathrm{ml})$ in the autologous bone group. Bleeds in excess of $500 \mathrm{ml}$ in three cases were treated with blood transfusion (Analysis 4.2).

\section{Knee range of movement}

Bucholz 1989 reported that all but 2 of the 40 participants (one in each group) attained greater than 90 degrees of knee flexion. Heikkilä 2011 provided data showing that the groups were similar with regard to knee stability and range of movement. At 12 months follow-up, Russell 2008 reported that greater proportions of participants in the bone cement group achieved full extension (59/67 versus $18 / 26$; RR $1.14,95 \%$ CI 0.91 to 1.44 ) and full 
flexion (59/67 versus $18 / 26$; RR $1.27,95 \%$ CI 0.97 to 1.67 ) than in the autograft group; Analysis 4.3.

\section{Return to pre-injury activity level}

None of the trials reported on return to pre-injury activity level. However, Bucholz 1989 reported that all 40 participants returned to their pre-injury employment status.

\section{Residual deformity and malunion}

As noted above, Bucholz 1989 reported loss of reduction in four participants (1/20 hydroxyapatite versus 3/20 autograft) (Analysis 4.4). During the radiographic examination at final follow-up (range of 6 to 66 months) in Bucholz 1989, the mean articular surface depression was $0.5 \mathrm{~mm}$ in the hydroxyapatite group versus $2.0 \mathrm{~mm}$ in the autograft group (formal statistical testing was not conducted). The mean joint line (articular) depression was around $3 \mathrm{~mm}$ in both groups at both 3 and 12 months in Heikkilä 2011. Russell 2008 found a lower rate of radiographic articular subsidence $(\geq 2 \mathrm{~mm})$ in the bone substitute group (6/69 versus $10 / 33$; RR $0.29,95 \%$ CI 0.11 to 0.72 ; Analysis 4.4 ).

Radiologically-assessed alignment of the injured limb was similar in the two groups of Heikkilä 2011 (mean 1.8 versus 3.1 degrees; MD - $1.30,95 \%$ CI -4.84 to 2.24; 25 participants; Analysis 4.5). Russell 2008 reported one case of varus malunion in each group (Analysis 4.4).

At 11 year follow-up, Heikkilä 2011 reported a lower incidence of radiologically diagnosed osteoarthritis in the affected knee in the bioactive glass group (3/5 versus $8 / 10$; RR 0.75 , $95 \%$ CI 0.34 to 1.64; Analysis 4.4).

\section{Time to radiological union}

All fractures united within three months in Russell 2008. The other two trials did not report this outcome.

\section{Other secondary outcomes}

None of the trials reported on pain measures, return to pre-injury activity levels, hospital length stay, patient satisfaction with treatment or total length of treatment.

\section{DISCUSSION}

\section{Summary of main results}

We included six trials in the review, involving a total of 429 adult participants and 431 tibial plateau fractures. The overall mean age of participants in this review was 42 years, and the majority were male (63\%). Three trials compared different methods of surgical fixation, and three compared three different types of bone substitute versus autologous bone graft. All of the included studies were small and assessed to be at substantial risk of bias, particularly due to lack of blinding.

\section{Circular external fixation with limited internal fixation (hybrid fixation) versus open reduction and internal fixation}

A five-centre trial conducted in Canada comparing hybrid fixation with standard open reduction and internal fixation (ORIF) reported results at 24 months for 66 participants with open or closed Schatzker types V or VI tibial plateau fractures (COTS 2006); we present the main results for this comparison in the Summary of findings for the main comparison. The quality of the evidence was rated low or very low quality for individual outcomes. The results for quality of life (assessed using the SF-36, but reported only for eight individual domains) tended to favour hybrid fixation, but the CIs for each domain also crossed the line of no effect, and we could not rule out a smaller benefit of ORIF. A similar finding applied to the Hospital for Special Surgery (HSS) scores and for WOMAC function scores at 24 months. Although the HSS results at six months of the hybrid fixation were better and may have represented a clinically important benefit relative to ORIF, the same did not apply to WOMAC function scores at six months. Based on the risk of 450 per 1000 people having at least one unplanned reoperation in the ORIF group, 99 fewer people per 1000 had an unplanned reoperation after hybrid fixation (95\% CI 197 fewer to 144 more). More participants in the ORIF group had multiple reoperations, which explains the significantly longer length of hospital stay in this group (mean 9.9 hybrid fixation versus 23.4 days ORIF; MD -13.50 days, $95 \%$ CI -14.77 to -12.23 days). Results of the two groups were comparable for the WOMAC pain subscale. Although mean knee range of motion values were higher in the hybrid group, the clinical benefit was uncertain and, notably, the two groups had comparable WOMAC stiffness scores. Based on a low return to pre-injury activity level in the ORIF group of 121 per 1000 people, 182 more people per 1000 had returned to their pre-injury activity level in the hybrid fixation group (95\% CI 15 fewer to 748 more).

\section{Less invasive stabilisation system (LISS) versus double-plating open reduction internal fixation}

A single-centre trial conducted in China comparing LISS versus double-plating ORIF reported results at 24 months in 84 people who had open or closed bicondylar tibial plateau fractures (Jaing 2008). Of note is that nearly twice as many participants (22 versus 12 ) in the double-plating group had a bone graft. The quality of the evidence was rated very low quality for all outcomes. The trial did not report quality of life, and there was no evidence of differences between the two groups in HSS knee scores. There was no 
difference between the two groups in individual complications or reoperation entailing implant removal or revision fixation. There were no data for pain, knee range of motion or return to pre-injury activity.

\section{Arthroscopically assisted percutaneous reduction and internal fixation versus standard open reduction and internal fixation}

A single-centre quasi-randomised trial conducted in China comparing arthroscopically assisted percutaneous reduction and internal fixation versus standard ORIF reported results at 14 months in 58 people who had closed Schatzker types II or III tibial plateau fractures (Shen 2011). The quality of the evidence was rated to be very low for all outcomes. Quality of life was not reported in this trial. There was very low quality evidence of higher HSS knee scores in the arthroscopically assisted group. No serious adverse events resulting the need for a reoperation were recorded; all five participants (2/38 in the arthroscopically assisted group versus 3/ 20 in the ORIF group) had relief from knee stiffness after receiving an intra-articular injection. The arthroscopically assisted group had a greater knee range of motion values. There were no data for pain or return to pre-injury activity.

\section{Bone substitute versus autologous bone grafts for filling bony voids}

Three trials compared three different bone substitutes with autologous bone grafts (Bucholz 1989; Heikkilä 2011; Russell 2008). Bucholz 1989, a quasi-randomised single centre trial conducted in the USA, reported the results for interporous hydroxyapatite up to 15 months in 40 participants who had closed unstable tibial plateau fractures. Heikkilä 2011, a single-centre trial conducted in Finland, reported the results for bioactive glass up to 12 months in 25 participants who had closed unilateral tibial plateau fractures. Eleven-year follow-up results were also available for 15 participants. Russell 2008, a 12-centre trial conducted in the USA, reported the main results for an endothermic calcium phosphate cement at 12 months in 92 participants (93 fractures) who had closed unstable tibial plateau fractures (Schatzker types I to VI). It was only possible to pool infection data. The quality of the evidence was rated very low for all outcomes. Quality of life and knee function score data were not available for any of the three trials. One trial (25 participants) found similar proportions of participants in the two groups with excellent or good lower limb function expressed in terms of ability to walk, climb stairs, squat and jump. The incidences of individual complications were similar in the bone substitute and autograft groups in all three trials. One trial found no cases of inflammatory response to the bone substitute. Two trials found no complications associated with the donor site in the autograft group. However, all participants in the autologous iliac bone graft group of Russell 2008 had postoperative pain from the harvest site that took 6 to 12 months to resolve. Otherwise, the three trials did not report pain or return to pre-injury levels of activity. Two trials found comparable results for range of motion in the two groups. In contrast, greater proportions of participants in the bone cement group achieved full extension and full flexion in Russell 2008; however, since the confidence intervals crossed the line of no effect, we could not rule out a smaller benefit in the autograft group. Two of the three trials found better joint line alignment in the bone substitute group, whereas the results in the two groups were similar in the third trial. One trial found fewer cases of radiologically detected osteoarthritis at 11 years follow-up in the bone substitute group.

\section{Overall completeness and applicability of evidence}

The evidence base comprises three single-trial comparisons and one three-trial comparison for which only very limited pooling is available. The evidence base is further weakened by the lack of data for many outcomes, in particular for quality of life and knee function scores.

Fracture type is a major factor when considering the applicability of the findings of the individual trials and comparisons. Thus it is important to note the restriction of the fracture population in COTS 2006 to the Schatzker types V and V, and that of Shen 2011 to the less complex Schatzker types II and III. Additionally, COTS 2006 included open and closed fractures, whereas Shen 2011 included closed fractures only. Both these are compatible with the perceived indications of hybrid fixation and percutaneous fixation, respectively (see How the intervention might work).

Contributing to the high risk of 'other' bias in two trials were concerns about performance bias based on other procedures, especially in the imbalance in the use of bone grafts in Jaing 2008, and the lack of details and control of fixation methods in Shen 2011. Lack of details of the interventions and differences in other procedures, including those for soft-tissue knee injuries, also have implications for applicability. The authors of COTS 2006 acknowledged that a major limitation of using percutaneous reduction and circular fracture fixation was the limited ability to assess and treat intraarticular soft-tissue damage, such as meniscal tears. Such lesions are easier to repair using a formal open reduction approach, but whether this results in a superior outcome, with lower risk of posttraumatic osteoarthritis, is not known. The availability of the interventions under test should also be considered; while the level of the intervention in our comparison was at bone substitutes, we are unsure whether any of the three specific compounds under test are now available for general use.

Only COTS 2006 reported on the return to pre-injury activity levels, finding a maximum return of $30 \%$ in the hybrid fixation group at 24 months. Given that most of the trial populations were of working age, more focus on economic outcomes such as return to work, duration of sick leave and length of hospital stay appears 
warranted. These data were not reported, with the exception of COTS 2006, where length of hospital stay was significantly shorter in the hybrid fixation group, and Bucholz 1989, which reported that all 40 participants returned to their pre-injury employment status. The problems of attrition bias for long-term outcomes are illustrated in Heikkilä 2011, but it is still likely that 24 month follow-up is insufficient to assess whether there is a difference in the incidence of osteoarthritis between the interventions.

\section{Quality of the evidence}

All six trials were at high risk of performance and detection biases relating to the lack of blinding, and two were at high risk of selection bias because they were quasi-randomised. Thus in our assessment of the quality of the evidence using GRADE, we downgraded the evidence for all reported outcomes for each comparison by one or two levels for these and other study limitations. We downgraded all outcomes one or two levels for imprecision given the small sample sizes and wide confidence intervals. The reasons for downgrading of the evidence for the comparison of hybrid fixation versus ORIF are presented in full in 'Summary of findings for the main comparison'. Another reason for downgrading in this comparison was for indirectness, relating to the unsatisfactory nature of some outcome measures. We thus judged the quality of most of the available evidence to be very low, meaning that we are very uncertain about the estimates.

Only one study acknowledged the role of industry in the funding and support for the trials undertaken (COTS 2006). In this trial, the authors acknowledged that a company that manufactures orthopaedic fracture fixation devices supported the research or preparation of the final paper. However, a clear statement was made that none of the authors received direct payment, other benefits or any commitment to deliver these from a commercial entity. None of the other papers contained such an acknowledgement or declaration. Accordingly, it remains unknown whether the support provided by industry had an impact on the conduct and reporting of the results in these.

\section{Potential biases in the review process}

Although our search was comprehensive and conducted in a rigorous way, it is still possible that we failed to identify studies, such as those reported only in conferences proceedings. Some changes to our protocol occurred after its publication. We have described these, including giving justification for these changes.

\section{Agreements and disagreements with other studies or reviews}

Mahadeva 2008 performed a systematic review of the literature around the use of open reduction and internal fixation versus hy- brid fixation for Shatzker type VI fractures, with similar results as those presented here. They found five studies relating to the topic area, of which only one was a multicentre RCT (COTS 2006). Of the others, two were biomechanical studies, and two were retrospective analyses. On the basis of the available evidence, the authors concluded that there was insufficient evidence to compare the techniques.

We have not identified any other systematic reviews published in this area, either comparing different types of surgical techniques or the use of different types of bone substitute material.

\section{AUTHORS' CONCLUSIONS Implications for practice}

Currently, there is insufficient evidence to ascertain the best method of fixation or the best method of addressing bone defects at the time of surgery. The current evidence, however, does not contradict the use of approaches aiming to limit the extent of softtissue dissection and damage nor the use of bone substitutes to avoid autograft donor site pain and complications.

\section{Implications for research}

Given the deficiencies in the current evidence, further research in the form of good quality, adequately-powered, multi-centre RCTs is warranted to inform surgical decisions for treating these fractures. Such trials should collect validated quality of life and patient-reported measures of lower-limb and knee function, adverse events and their management, and they should also conduct an economic evaluation that includes both direct (health service) and indirect costs. A minimum follow-up of two years is required. Such research should be preceded by research that aims to identify priority questions and the most appropriate outcome measures for these injuries. Decisions on prioritisation should take into account the coverage of the current evidence base as well as the topics covered by the ongoing trials. In our view, the focus should be on methods of fixation rather than bone substitutes for bone defects. Prior subgrouping by fracture categories is also important. All research, including that conducted by industry, should be reported in full in a timely way and according to the CONSORT statement for non-pharmacologic treatment trials.

\section{ACKNOWLEDGEMENTS}

We thank Xavier Griffin, Helen Handoll, Mario Lenza, Steven Olson and Marc Swiontkowski for their helpful feedback during the editorial review process of the protocol and review. We thank Lindsey Elstub for her assistance in the preparation of this review and Joanne Elliott for her assistance in the search strategies. 
This project was supported by the National Institute for Health Research via Cochrane Infrastructure funding to the Cochrane Bone, Joint and Muscle Trauma Group. The views and opinions expressed therein are those of the authors and do not necessarily reflect those of the Systematic Reviews Programme, NIHR, NHS or the Department of Health.

\section{R E F E R E N C E S}

\section{References to studies included in this review}

Bucholz 1989 \{published data only\}

Bucholz RW, Carlton A, Holmes R. Interporous hydroxyapatite as a bone graft substitute in tibial plateau fractures. Clinical Orthopaedics and Related Research 1989; (240):53-62.

COTS 2006 \{published data only\}

* Canadian Orthopaedic Trauma Society. Open reduction and internal fixation compared with circular fixator application for bicondylar tibial plateau fractures. Results of a multicenter, prospective, randomized clinical trial. Journal of Bone and Joint Surgery - American Volume 2006;88(12): 2613-23.

Hall JA, Beuerlein MJ, Mckee MD, Canadian Orthopaedic Trauma Society. Open reduction and internal fixation compared with circular fixator application for bicondylar tibial plateau fractures. Surgical technique. Journal of Bone and Joint Surgery - American Volume 2009;91(Suppl 2): 74-88.

Pirani S, McKee M, The Canadian Orthopaedic Trauma Society. Randomized trial of bicondylar tibial plateau fractures treated with $\mathrm{AO}$ or ring fixator methods: early reintervention rates for complications. Journal of Bone and Joint Surgery - British Volume 2008;90 (Suppl 1):47.

Heikkilä 2011 \{published data only\}

* Heikkilä JT, Kukkonen J, Aho AJ, Moisander S, Kyyrönen T, Mattila K. Bioactive glass granules: a suitable bone substitute material in the operative treatment of depressed lateral tibial plateau fractures: a prospective, randomized 1 year follow-up study. Journal of Materials Science. Materials in Medicine 2011;22(4):1073-80.

Pernaa K, Koski I, Mattila K, Gullichsen E, Heikkilä J, Aho A, et al. Bioactive glass S53P4 and autograft bone in treatment of depressed tibial plateau fractures - a prospective randomized 11-year follow-up. Journal of Long-Term Effects of Medical Implants 2011;21(2):139-48.

Jaing 2008 \{published data only\}

Jiang R, Luo C-F, Wang M-C, Yang T-Y, Zeng B-F. A comparative study of Less Invasive Stabilization System (LISS) fixation and two-incision double plating for the treatment of bicondylar tibial plateau fractures. Knee 2008; 15(2):139-43.

Russell 2008 \{published data only\}

Russell TA, Leighton RK, Alpha-BSM Tibial Plateau Fracture Study Group. Comparison of autogenous bone graft and endothermic calcium phosphate cement for defect augmentation in tibial plateau fractures. A multicenter, prospective, randomized study. Journal of Bone \& Joint Surgery - American Volume 2008;90(10):2057-61.

Shen 2011 \{published data only\}

Shen G, Zhou J. Comparison study on effectiveness between arthroscopy assisted percutaneous internal fixation and open reduction and internal fixation for Schatzker types II and III tibial plateau fractures. Chinese Journal of Reparative and Reconstructive Surgery 2011;25(10):1201-4.

\section{References to studies excluded from this review}

\section{Carter 1996 \{published data only\}}

Carter AT, Uri RL, Goldstock L, Lozman J. Hybrid external fixation for bicondylar tibial plateau fractures: a comparison to double-buttress plating. Orthopaedic Transactions 1996; 20(4): 1076.

Chan 2012 \{published data only\}

Chan CC, Keating J. Comparison of outcomes of operatively treated bicondylar tibial plateau fractures by external fixation and internal fixation. Malaysian Orthopaedic Journal 2012;6(1):7-12.

Dall'oca 2012 \{published data only\}

Dall'oca C, Maluta T, Lavini F, Bondi M, Micheloni G, Bartolozzi P. Tibial plateau fractures: compared outcomes between ARIF and ORIF. Strategies in Trauma and Limb Reconstruction 2012;7(3):163-75.

Egol 2004b \{published data only\}

Egol K, Tajwani N, Sims S, Kummer F, Koval K. Treatment of complex tibial plateau fractures using the less invasive stabilization system plate: clinical experience and a laboratory comparison with double plating. Journal of Trauma 2004;57(2):340-6.

Gunther 2008 \{published data only\}

Gunther T, Horvath B, Almasi J. Minimal invasive operative treatment of the tibial plateau fractures with the help of the arthroscope and fluoroscope. European Journal of Trauma and Emergency Surgery 2008;34(Suppl 1):150-1.

Hansen 2009 \{published data only\} Hansen M, Attal RE, Blum J, Blauth M, Rommens PM. Intramedullary nailing of the tibia with the expert tibia nail [Tibiamarknagelung mit dem Expert Tibia Nagel]. Operative Orthopadie und Traumatologie 2009;21(6): 620-35. 
Jubel 2004 \{published data only\}

Jubel A, Andermahr J, Mairhofer J, Prokop A, Hahn U, Rehm KE. Use of the injectable bone cement Norian SRS for tibial plateau fractures. Results of a prospective 30month follow-up study [Die anwendung des injizierbaren knochenzements Norian SRS bei tibiakopffrakturen. Ergebnisse einer prospektiven anwendungsbeobachtung mit einem follow-up von 30 monaten]. Der Orthopäde 2004; 33(8):919-27.

Krupp 2009 \{published data only\}

Krupp RJ, Malkani AL, Roberts CS, Seligson D, Crawford $\mathrm{CH}$ 3rd, Smith L. Treatment of bicondylar tibia plateau fractures using locked plating versus external fixation. Orthopedics 2009;32(8):559-66

Lobenhoffer 1999 \{published data only\}

Lobenhoffer P, Schulze M, Gerich T, Lattermann C, Tscherne $\mathrm{H}$. Closed reduction/percutaneous fixation of tibial plateau fractures: arthroscopic versus fluoroscopic control of reduction. Journal of Orthopaedic Trauma 1999; 13(6):426-31.

Malakasi 2013 \{published data only\}

Malakasi A, Lallos S, Chronopoulos E, Korres DS, Efstathopoulos NE. Comparative study of internal and hybrid external fixation in tibial condylar fractures. European Journal of Orthopaedic Surgery and Traumatology 2013;23(1):97-103.

\section{NCT00429585 \{published data only\}}

Tornetta P. A multicenter randomized trial comparing IM nails and plate fixation in proximal tibial fractures. http://clinicaltrials.gov/show/NCT00429585 (accessed 15 September 2014).

\section{NCT00610701 \{published data only\}}

Crist B. Lateral versus anterior spanning external fixator for tibial plateau fractures. clinicaltrials.gov/ct2/show/ NCT00610701 (accessed 15 September 2014).

Ohdera 2003 \{published data only\}

Ohdera T, Tokunaga M, Hiroshima S, Yoshimoto E, Tokunaga J, Kobayashi A. Arthroscopic management of tibial plateau fractures-comparison with open reduction method. Archives of Orthopaedic and Trauma Surgery 2003; 123(9):489-93.

Ong 2012 \{published data only\}

Ong JC, Kennedy MT, Mitra A, Harty J. Fixation of tibial plateau fractures with synthetic bone graft versus natural bone graft: a comparison study. Irish Journal of Medical Science 2012;181(2):247-52.

Sung 2008 \{published data only\}

Sung JK, Levin R, Siegel J, EinhornTA, Creevy WR, Tornetta P. Reuse of external fixation components: a randomized trial. Journal of Orthopaedic Trauma 2008;22 (2):126-30.

Tahririan 2014 \{published data only\} Tahririan MA, Mousavitadi SH, Derakhshan M. Comparison of functional outcomes of tibial plateau fractures treated with nonlocking and locking plate fixations: a nonrandomized clinical trial. ISRN Orthopedics 2014;2014:324573. [DOI: 10.1155/2014/324573]

Weaver 2012 \{published data only\}

Weaver MJ, Harris M, Strom A, Smith R, Lhowe D, Zurakowski D, et al. Fracture pattern and fixation type related to loss of reduction in bicondylar tibial plateau fractures. Injury 2012;43(6):864-9.

Zhang 2012 \{published data only\}

Zhang Y, Fan D, Ma B, Sun G. Treatment of complicated tibial plateau fractures with dual plating via a 2 -incision technique. Orthopedics 2012;35(3):359-64.

Zhuo 2008 \{published data only\} Zhuo N, Li Z, Ge J, Lu X. Comparative study on internal fixation and external fixation for the treatment of complex tibial plateau fracture. Chinese Journal of Reparative and Reconstructive Surgery 2008;22(8):952-5.

\section{References to studies awaiting assessment}

Jordan 2014 \{published data only\} Jordan R, Hao J, Fader R, Gibula D, Mauffrey C. Study protocol: trial of inflation osteoplasty in the management of tibial plateau fractures. European Journal of Orthopaedic Surgery and Traumatology 2014;24(5):647-53.

\section{References to ongoing studies}

Kuros Biosurgery AG \{published data only\}

* Kuros Biosurgery AG. A phase 2 randomized, controlled, open-label (dose-blinded), multi-center, dose-finding study of the safety and efficacy of I-0401 in the treatment of patients with fractures of the tibial plateau requiring grafting. apps.who.int/trialsearch/Trial2.aspx?TrialID= EUCTR2006-003688-30-GB (accessed 27 July 2015). Messmer P. Safety and efficacy of I 0401 in the treatment of tibial plateau fractures requiring grafting. clinicaltrials.gov/ ct2/show/NCT00409799 (accessed 15 September 2014).

NCT01828905 \{published data only\}

* Nusselt T, Hofmann A, Wachtlin D, Gorbulev S, Rommens PM. CERAMENT treatment of fracture defects (CERTiFy): protocol for a prospective, multicenter, randomized study investigating the use of CERAMENTTM bone void filler in tibial plateau fractures. Trials 2014;15: 75. [DOI: $10.1186 / 1745-6215-15-75$ ]

Rommens PM, Hofmann A. Cerament treatment of fracture defects (CERTiFy). clinicaltrials.gov/ct2/show/ NCT01828905 (accessed 15 September 2014).

\section{NCT02035917 \{published data only\}}

Tahririan MA, Derakhshan M. Comparison of tibial plateau fractures outcomes treated with non-locking and locking plate. clinicaltrials.gov/ct2/show/NCT02035917 (accessed 15 September 2014).

\section{Additional references}

\section{Barei 2006}

Barei DP, Nork SE, Mills WJ, Coles CP, Henley MB, Benirschke SK. Functional outcomes of severe bicondylar 
tibial plateau fractures treated with dual incisions and medial and lateral plates. Journal of Bone \& Joint Surgery American Volume 2006;88(8):1713-21.

\section{Bennett 1994}

Bennett WF, Browner B. Tibial plateau fractures: a study of associated soft tissue injuries. Journal of Orthopaedic Trauma 1994;8(3):183-8.

\section{Binkley 1999}

Binkley JM, Stratford PW, Lott SA, Riddle DL. The Lower Extremity Functional Scale (LEFS): scale development, measurement properties, and clinical application. North American Orthopaedic Rehabilitation Research Network. Physical Therapy 1999;79(4):371-83.

\section{Court-Brown 1990}

Court-Brown CM, Wheelwright EF, Christie J, McQueen MM. External fixation for type III open tibial fractures. Journal of Bone \& Joint Surgery - British Volume 1990;72(5): $801-4$.

\section{Dawson 1998}

Dawson J, Fitzpatrick R, Murray D, Carr A. Questionnaire on the perceptions of patients about total knee replacement. Journal of Bone and Joint Surgery - British Volume 1998;80 (1):63-9.

\section{Deeks 2011}

Deeks JJ, Higgins JPT, Altman DG on behalf of the Cochrane Statistical Methods Group. Chapter 9: Analysing data and understanding meta-analyses. In: Higgins JPT, Green S (editors). Cochrane Handbook for Systematic Reviews of Interventions Version 5.1.0 (updated March 2011). The Cochrane Collaboration, 2011. Available from www.cochrane-handbook.org.

\section{Dirschl 1997}

Dirschl DR, Dahners LE. Current treatment of tibial plateau fractures. Journal of the Southern Orthopaedic Association 1997;6(1):54-61. [PUBMED: 9090625]

\section{Egol 2004a}

Egol KA, Su E, Tejwani NC, Sims SH, Kummer FJ, Koval $\mathrm{KJ}$. Treatment of complex tibial plateau fractures using the less invasive stabilization system plate: clinical experience and a laboratory comparison with double plating. Journal of Trauma-Injury Infection \& Critical Care 2004;57(2):340-6.

\section{Higgins 2011a}

Higgins JPT, Green S (editors). Cochrane Handbook for Systematic Reviews of Interventions Version 5.1.0 [updated March 2011]. The Cochrane Collaboration, 2011. Available from www.cochrane-handbook.org.

\section{Higgins 2011b}

Higgins JPT, Altman DG, Sterne JAC (editors). Chapter 8: Assessing risk of bias in included studies. In: Higgins JPT, Green S (editors). Cochrane Handbook for Systematic Reviews of Interventions Version 5.1.0 (updated March 2011). The Cochrane Collaboration, 2011. Available from www.cochrane-handbook.org.

\section{Howard 1997}

Howard M, Court-Brown CM. Epidemiology and management of open fractures of the lower limb. British Journal of Hospital Medicine 1997;57(11):582-7.

\section{Insall 1989}

Insall JN, Dorr LD, Scott RD, Scott WN. Rationale of the Knee Society clinical rating system. Clinical Orthopaedics and Related Research 1989;(248):13-14.

\section{Kayali 2008}

Kayali C, Oztürk H, Altay T, Reisoglu A, Agus H. Arthroscopically assisted percutaneous osteosynthesis of lateral tibial plateau fractures. Canadian Journal of Surgery 2008;51(5):378-82.

\section{Kumar 2000}

Kumar A, Whittle AP. Treatment of complex (Schatzker Type VI) fractures of the tibial plateau with circular wire external fixation: retrospective case review. Journal of Orthopaedic Trauma 2000;14(5):339-44.

\section{Laflamme 2003}

Laflamme GY, Heimlich D, Stephen D, Kreder HJ, Whyne CM. Proximal tibial fracture stability with intramedullary nail fixation using oblique interlocking screws. Journal of Orthopaedic Trauma 2003;17(7):496-502.

\section{Lefebvre 2011}

Lefebvre C, Manheimer E, Glanville J. Chapter 6: Searching for studies [Box 6.4]. In: Higgins JPT, Green $S$ (editors). Cochrane Handbook for Systematic Reviews of Interventions Version 5.1.0 (updated March 2011). The Cochrane Collaboration, 2011. Available from www.cochrane-handbook.org.

\section{Mahadeva 2008}

Mahadeva D, Costa M L, Gaffey A. Open reduction and internal fixation versus hybrid fixation for bicondylar/severe tibial plateau fractures: a systematic review of the literature. Archives of Orthopaedic and Trauma Surgery 2008;128(10): 1169-75.

\section{Mallik 1992}

Mallik AR, Covall DJ, Whitelaw GP. Internal versus external fixation of bicondylar tibial plateau fractures. Orthopaedic Review 1992;21(12):1433-6.

\section{Moore 1987}

Moore TM, Patzakis MJ, Harvey JP. Tibial plateau fractures: definition, demographics, treatment rationale, and longterm results of closed traction management or operative reduction. Journal of Orthopaedic Trauma 1987;1(2): 97-119.

\section{OTA 1996}

Orthopaedic Trauma Association Committee for Coding and Classification. Fracture and dislocation compendium. Journal of Orthopaedic Trauma 1996; Vol. 10, issue Suppl $1: 31-4$.

\section{Piper 2005}

Piper KJ, Won HY, Ellis AM. Hybrid external fixation in complex tibial plateau and plafond fractures: an Australian audit of outcomes. Injury 2005;36(1):178-84. 
RevMan

The Nordic Cochrane Centre, The Cochrane Collaboration. Review Manager (RevMan). 5.3. Copenhagen: The Nordic Cochrane Centre, The Cochrane Collaboration, 2014.

\section{Schatzker 1979}

Schatzker J, McBroom R, Bruce D. The tibial plateau

fracture. The Toronto experience 1968- 1975. Clinical Orthopaedics \& Related Research 1979, (138):94-104.

\section{Shepherd 2002}

Shepherd L, Abdollahi K, Lee J, Vangsness CT Jr. The prevalence of soft tissue injuries in nonoperative tibial plateau fractures as determined by magnetic resonance imaging. Journal of Orthopaedic Trauma 2002;16(9): 628-31.

\section{Sterne 2011}

Sterne JAC, Egger M, Moher D on behalf of the Cochrane Bias Methods Group. Chapter 10: Addressing reporting biases. In: Higgins JPT, Green S (editors). Cochrane Handbook for Systematic Reviews of Interventions Version 5.1.0 (updated March 2011). The Cochrane Collaboration, 2011. Available from www.cochrane-handbook.org.

Ware 1992

Ware JE, Sherbourne CD. The MOS 36-item short-form health survey (SF36). I. Conceptual framework and item selection. Medical Care 1992;30(6):473-83.

\section{Watson 2002}

Watson JT, Ripple S, Hoshaw SJ, Fyhrie D. Hybrid external fixation for tibial plateau fractures: clinical and biomechanical correlation. Orthopedic Clinics of North America 2002;33(1):199-209, ix.

\section{WOMAC}

The Western Ontario and McMaster Universities Osteoarthritis Index (WOMAC). http://www.womac.org (accessed 15 September 2014).

\section{Young 1994}

Young MJ, Barrack RL. Complications of internal fixation of tibial plateau fractures. Orthopaedic Review 1994;23: $149-54$.

\section{References to other published versions of this review}

\section{McNamara 2012}

McNamara IR, Hing CB, Smith TO, Clark AB, Nielsen DM, Donell S. Surgical fixation methods for tibial plateau fractures. Cochrane Database of Systematic Reviews 2012,

Issue 3. [DOI: 10.1002/14651858.CD009679]

* Indicates the major publication for the study 


\section{CHARACTERISTICS OFSTUDIES}

\section{Characteristics of included studies [ordered by study ID]}

\section{Bucholz 1989}

Methods

Participants
Single-centre, quasi-randomised controlled trial

Country of study: United States

Sample size: 49 participants ( 9 participants lost to follow-up were excluded)

Age: (of 40 ) mean 37 years, range 16 to 73 years

Gender: (of 40) 24 males (60\%) and 16 females

Fracture type: all presented with closed tibial plateau fractures, which were categorised according to the Hohl classification. Of 40 fractures, there were 22 lateral split depression fractures, 8 medial split depression fractures, 6 bicondylar fractures and 4 isolated depression fractures

Injury severity: injury severity score not reported; 23 (58\%) were from traffic accidents Inclusion criteria: Inclusion criteria included knees that were deemed unstable secondary to medial and lateral fractures, depression of the articular surface of more than $10 \mathrm{~mm}$ or translation of the condylar fragments of greater than $5 \mathrm{~mm}$ in mediolateral direction

Exclusion criteria: not specifically described

Interventions

Surgical procedure: The fracture was exposed via the 'standard surgical incisions' and the osteochondral fragments of the plateau were elevated. Once articular congruity had been restored, the dimensions of the cavity defect were measured. Fixation was achieved by standard plate and screw fixation in both groups

Bone filler 1: Hydroxyapatite bone substitute $(n=20)$ : the defect size was measured, and a block that had been sized to gain a press fit with the surrounding bone was inserted into the defect.

Bone filler 2: autologous bone graft ( $n=20)$ : an appropriate volume of cancellous graft was harvested from the ipsilateral iliac crest and impacted tightly into the metaphyseal defect

Outcomes

Follow-up: 4 to 6 week periods until fracture union and knee rehabilitation were complete. The autograft group was followed up for an average of 15.4 months (range 6 to 46 months) postsurgery. The hydroxyapatite group was followed up for an average of 34 . 5 months (range 12 to 66 months) postsurgery. Follow-up was performed by physical examination and radiological means looking at residual joint depression

Outcome measures: radiographic (initial reduction of articular surface, obliteration of all major fracture lines, subsequent settling of the articular surface at final follow-up, maintenance of joint-space height over the fracture and appearance of the hydroxyapatite implant), knee range of movement and pain, return to pre-injury employment, complications and their management

Notes

$-$

\section{Risk of bias}


Bucholz 1989 (Continued)

\begin{tabular}{l|l|l}
$\begin{array}{l}\text { Random sequence generation (selection } \\
\text { bias) }\end{array}$ & High risk & $\begin{array}{l}\text { Quasi-randomisation performed "based } \\
\text { solely on which day of the week the patients } \\
\text { presented themselves to the admitting ser- } \\
\text { vice" (page 53) }\end{array}$ \\
\hline $\begin{array}{l}\text { Allocation concealment (selection bias) } \\
\begin{array}{l}\text { Blinding of participants and personnel } \\
\text { (performance bias) } \\
\text { All outcomes }\end{array}\end{array}$ & High risk & Quasi-randomised and thus not concealed. \\
\hline
\end{tabular}

Blinding of outcome assessment (detection High risk

bias)

There was no mention of blinding.

All outcomes

Incomplete outcome data (attrition bias) High risk All outcomes

igh risk

\begin{tabular}{|c|c|c|}
\hline & & cation groups were not stated \\
\hline Selective reporting (reporting bias) & Unclear risk & $\begin{array}{l}\text { All outcomes reported as being collected } \\
\text { were reported in the 'Results' section. No } \\
\text { reference to a published protocol or trial } \\
\text { registration number was provided and none } \\
\text { was found }\end{array}$ \\
\hline
\end{tabular}

\begin{tabular}{ll}
\hline Other bias & Unclear risk
\end{tabular}

Although there were similarities in the demographic characteristics of the two groups for the 40 participants included in the analyses, there were no baseline data for the 9 participants lost to follow-up nor for fracture type. Additionally, management of other injuries such as ipsilateral femur fracture and ACL ruptures was not described

COTS 2006

\begin{tabular}{ll}
\hline Methods & 5-centre RCT \\
\hline Participants & Country of study: Canada \\
& Sample size: 82 participants with 83 fractures \\
& Age: mean 45 years \\
& Gender: 52 males (63\%) and 30 females \\
& Fracture type: open or closed displaced bicondylar tibial plateau fractures classified as \\
& Schatzker types V (18 fractures) or VI (65 fractures) and OTA types C1 (20 fractures), \\
& C2 (39 fractures) or C3 (24 fractures) \\
& Injury severity: mean injury severity score (0 to 75; most severe) was 8.2 ; there were 14 \\
& open injuries; 44 (54\%) were from traffic (including bicycle) accidents \\
& Inclusion criteria: skeletally mature and below the age of 65 years, presenting with
\end{tabular}

Surgical fixation methods for tibial plateau fractures (Review) 
displaced bicondylar tibial plateau fracture (Schatzker type V and VI and OTA types $\mathrm{C} 1, \mathrm{C} 2$, and C3), and with at least one of the following features: an intra-articular step or gap of $>2 \mathrm{~mm}$; extra-articular translation of $>1.0 \mathrm{~cm}$ or angulation of $>10^{\circ}$; an open fracture; compartment syndrome requiring fasciotomy; or an associated ligament injury requiring repair

Exclusion criteria: pathologic fracture; definitive surgery more than 14 days after the injury; a pre-existing joint disease; a severe systemic illness (active cancer, chemotherapy, insulin-dependent diabetes, renal failure, haemophilia, or a medical contraindication for surgery); open growth plates; a vascular injury requiring repair; or a severe head injury (initial Glasgow coma scale score of $<8$ ) or other neurological condition deemed to interfere with rehabilitation ticipant had bilateral injuries)). Participants were managed with a closed, percutaneous or limited open reduction of the articular surface followed by the insertion of percutaneous lag screws to stabilise the articular fragment(s); no plates were used. A femoral distractor and ligamentotaxis were used to reduce the fractures assisted by percutaneous techniques to elevate the fracture fragments. Subsequently a standard Ilizarov circular fixator was applied with a minimum of four points of purchase proximally and the shaft was reduced and fixation was applied through the distal rings of the frame. If proximal purchase, stability or both were poor, or a substantial knee ligament injury was present, a hinged frame that spanned the knee was then applied. No bone grafts were used All participants in the hybrid fixation were permitted to commence partial to full weightbearing (degree of weight-bearing dictated by surgeon) immediately after surgery. All participants were full weight-bearing at 12 weeks

Surgical procedure 2: ORIF group $(\mathrm{n}=40)$, standard AO principles of exposure and fixation were used. A single anterior incision or combined medial and lateral incisions (at the discretion of the treating surgeon) with arthrotomy were used to perform an open reduction and lag-screw fixation of the articular surface. Plates were applied medially and laterally to re-establish tibial alignment and buttress the articular repair. No locking plates were used in this series. Bone grafts could be used. Postoperatively, knees without a ligamentous or meniscal injury were placed in a removable knee immobiliser and permitted early motion. Weight-bearing was restricted (non-weight bearing) for the first 6 weeks. This was progressed to partial weight-bearing from 6 to 12 weeks postoperatively, and full weight-bearing initiated at 12 weeks

Follow-up: Participants were assessed preoperatively and completed outcome questionnaires, with radiographs at 6,12 and 24 months after the injury

Outcome measures: Short-Form 36 (SF-36) health status, Hospital for Special Surgery Knee score (HSS score), Western Ontario and McMaster Universities Osteoarthritis Index (WOMAC), complications resulting reoperation, the quality of radiographic reduction, return to pre-injury level of activity, range of knee motion, length of surgery, blood loss, presence of radiologically detected osteoarthritis and length of hospital stay where non-weight bearing was stipulated for the ORIF group, and those in the hybrid fixation group were permitted to bear weight (to some degree) immediately after surgery. However, intrasample variation may have been evident, as the level of weight-bearing was not controlled within the hybrid fixation group 
Randomisation undertaken on the morning of the planned operative intervention to allow preoperative frame construction. If randomisation was undertaken intraoperatively, the authors suggested that this would have "necessitated an unacceptable delay in the logistics of equipment assembly" (page 2615) and therefore would have been undesirable

\section{Risk of bias}

Bias

Random sequence generation (selection Low risk bias)

Allocation concealment (selection bias) Low risk
Participants were "randomised by sequentially numbered" sealed envelopes

\begin{tabular}{|l|l} 
search nurse who screened the participant performed randomi- \\
sation
\end{tabular}
Allocation was performed using "opaque, sealed envelopes". Re- sation

Blinding of participants and personnel High risk (performance bias)

There was no mention of blinding. Given the contrast in the All outcomes interventions, blinding is not possible for either the personnel or participants

Blinding of outcome assessment (detection High risk bias)

Blinding was not possible for outcome assessors since it is not practical to conceal a circular fixator

All outcomes

Incomplete outcome data (attrition bias) Unclear risk All outcomes

At 2 years, nine participants in the hybrid fixation group (21\%) and seven $(17.5 \%)$ in the ORIF group (one of whom had died) were lost to follow-up. Although losses were comparable between groups, these were still quite high. There was some potential mislabelling of SF-36 data and range of motion results

Selective reporting (reporting bias) Unclear risk

All outcome measurements outlined in the 'Methods' section were reported in the 'Results' section. No reference to a published protocol or trial registration number was provided and none was identified

Other bias

Low risk

The baseline characteristics of the two groups were comparable. No other risk of bias was identified

Heikkilä 2011

\begin{tabular}{ll}
\hline Methods & Single-centre RCT \\
\hline Participants & Country of study: Finland \\
& Sample size: 25 participants \\
& Age: mean 54 years, range 25 to 82 years \\
& Gender: 12 males (48\%) and 13 females \\
& Fracture type: closed depressed unilateral tibial comminuted plateau fracture (AO clas- \\
& sification 41 B2 and B3) \\
Injury severity: injury severity score not reported; unknown number resulted from traffic
\end{tabular}


Heikkilä 2011 (Continued)

accidents

Inclusion criteria: All patients were healthy, without systemic diseases and with normal bone structure. Joint line depression of more than $3 \mathrm{~mm}$ was indication for operative treatment

Exclusion criteria: not formally defined.

Interventions

Surgical procedure: The lateral condyle was exposed through an anterolateral posteriorly curved incision. Intact lateral meniscus and anterior cruciate ligament were observed in all knees. Lateral meniscus was detached at its tibial insertion to visualise the joint line. After lateral cortical fenestration, the articular surface was elevated under direct visual control. The fracture was then temporarily fixed using K-wires, and the elevation of articular surface was confirmed by preoperative plain films

Bone filler 1: Synthetic bone graft group $(n=14)$; granules were packed manually to quantitatively fill the defect caused by the fracture and the elevation

Bone filler 2: Autograft group ( $\mathrm{n}=11)$; defect was filled with autologous bone harvested from anterior iliac crest. The iliac crest was exposed using an incision along the superior iliac crest, which was chiseled temporarily aside, and medial lamina and corticocancellous bone chips were used as filler material

Outcomes

Follow-up: Clinical evaluation at 3 and 12 months postoperatively, and radiological evaluation immediately postoperatively then at 3 and 12 months. An 11 year follow-up of 15 participants is also reported

Outcome measures: Lower limb function, KOOS at 11 years, complications, range of knee motion, knee stability, blood loss, articular depression, presence of radiologically detected osteoarthritis

Notes

The demographics were not specified, but authors reported no statistical differences with regard to the fracture anatomy, surface area of the fracture or the degree of depression on condyle surface between groups

\section{Risk of bias}

Bias

Authors' judgement Support for judgement

Random sequence generation (selection Unclear risk bias)

The randomisation was reported as being "performed using closed envelopes". The method of sequence generation was not reported

Allocation concealment (selection bias) Unclear risk

The randomisation was reported as being "performed using closed envelopes". Insufficient description of methods to safeguard allocation concealment

Blinding of participants and personnel High risk (performance bias)

There was no mention of blinding. Given the contrast in the All outcomes interventions, it is unlikely that blinding was done

Blinding of outcome assessment (detection High risk bias)

There was no mention of blinding.

All outcomes

Surgical fixation methods for tibial plateau fractures (Review)

Copyright @ 2015 The Cochrane Collaboration. Published by John Wiley \& Sons, Ltd. 
Heikkilä 2011 (Continued)

\begin{tabular}{|c|c|c|}
\hline $\begin{array}{l}\text { Incomplete outcome data (attrition bias) } \\
\text { All outcomes }\end{array}$ & Unclear risk & $\begin{array}{l}\text { The abstract of the long term report of this trial refers to the } \\
\text { recruitment of } 29 \text { patients, but the main report only reports } \\
\text { results for } 25 \text {. Otherwise there was no reported loss to follow- } \\
\text { up in the main report. The loss to follow-up was unbalanced } \\
\text { at } 11 \text { year follow-up, putting this at high loss of attrition bias. } \\
\text { Incomplete outcome data }\end{array}$ \\
\hline
\end{tabular}

Selective reporting (reporting bias) High risk

All outcome measurements outlined in the 'Methods' section were reported in the 'Results' section, but numerical data were often not available. No reference to a published protocol or trial registration number was provided or found

Other bias $\quad$ Unclear risk

Baseline balance claimed, but data were not provided, and there was a 7 -year difference in the mean ages ( 57 years bioactive glass versus 50 years autograft). No other risk of bias was identified

Jaing 2008

Methods

Participants
Randomised clinical trial, single centre

Country of study: China

Sample size: 96 participants (12 were excluded, of which 8 were lost to follow-up)

Age: (of 84) mean 42 years

Gender: (of 84) 58 males (69\%) and 26 females

Fracture type: bicondylar tibial plateau fractures AO/OTA type C. Of 84: type C1, 13 fractures; type C2, 35 fractures; and type C3, 38 fractures

Injury severity: injury severity score not reported. However, 11 were open fractures (Gustilo type I, 1 fracture; type II, 7 fractures; type IIIA, 3 fractures), and the soft-tissue injuries for closed fractures were rated using Tscherne (grade 0, 29 fractures; grade I, 15 fractures; grade II, 22 fractures; grade III, 7 fractures). 54 (64\%) were high energy injuries

Inclusion criteria: unilateral fractures in participants which were independently ambulating before the injury; bicondylar tibial plateau fracture (AO/OTA type C)

Exclusion criteria: fractures older than 3 weeks prior to surgery; pathological fractures; open fractures requiring soft-tissue coverage or vascular repair; concomitant ipsilateral femoral fracture; participants requiring intensive care support

Interventions

Standardised surgical procedure elements: All participants had transcalcaneal skeletal traction. Participants with open wounds underwent surgical debridement within 8 hours of injury, with subsequent tetanus and intravenous antibiotics administered. Bone graft could be used in either intervention. Postoperatively, all participants from each group were permitted to start range-of-motion exercises and use a continuous passive motion machine once the incisions were sealed and dry. In cases of anterior cruciate ligament injury, a hinged brace was used for 12 to 16 weeks. All participants were non-weight bearing for the initial 12 postoperative weeks

Surgical procedure 1: Less invasive stabilisation system (LISS) group $(\mathrm{n}=41)$ were treated with a LISS plate (5 or 9 hole version) via an anterolateral incision alone

Surgical procedure 2: ORIF $(\mathrm{n}=43)$ : treated with dual-plate fixation via a anterolat- 
eral and posteromedial incision using anterolateral T- or L-shaped buttress plate plus a posteromedial limited contact dynamic compression plate

Outcomes

Follow-up: 12 and 24 months. A record was made of the time of fracture healing and if any postoperative complications occurred during normal clinical assessments

Outcome measures: Hospital Special Surgery (HSS) knee function score, fracture healing time, requirement for bone graft and postoperative complications including malreduction of articular surfaces, malalignment, and nonunion

Notes

Risk of bias

\begin{tabular}{ll|l} 
Bias & Authors' judgement & Support for judgement \\
\hline $\begin{array}{l}\text { Random sequence generation (selection } \\
\text { bias) }\end{array}$ & Low risk & $\begin{array}{l}\text { Randomisation was "accomplished with the } \\
\text { use of computer-generated random numbers" }\end{array}$ \\
\hline Allocation concealment (selection bias) & Unclear risk & $\begin{array}{l}\text { The location, administrator or any specific } \\
\text { methods for the randomisation procedure } \\
\text { were not stated. No mention was made of } \\
\text { methods for safeguarding allocation conceal- } \\
\text { ment }\end{array}$ \\
\hline
\end{tabular}

Blinding of participants and personnel High risk (performance bias)

All outcomes
There was no mention of blinding. Given the contrast in the interventions, this was not possible for the surgeons. Although the team of surgeons "had no preference for one particular method of fixation", the authors acknowledged that the participating surgeons "had little previous experience with LISS fixation"

The paper did not document whether the outcome assessors were blinded to the allocated fracture fixation device

bias)

All outcomes

Incomplete outcome data (attrition bias) High risk

All outcomes

Selective reporting (reporting bias)

Unclear risk
No allocation, baseline characteristics or follow-up data were provided for 12 (12.5\% of 96 randomised participants) postrandomisation exclusions (" 1 death, 3 comorbid psychological disorders and 8 lost to follow-up”)

All outcome measurements outlined in the 'Methods' section were reported in the 'Results' section. However, there was no reference to a published protocol or trial registration number, and none was found 
Jaing 2008 (Continued)

Other bias

High risk

Although the listed baseline characteristics were comparable for the 84 participants included in the analyses, there were greater numbers of meniscal repairs (19 versus 23), cruciate ligament surgeries (7 versus 11) and bone grafts (12 versus 22) in the double plating group, which can reflect differences in the injury characteristics, performance or both

Russell 2008

Methods

Participants 12-centres RCT

Country of study: United States

Sample size: 119 participants entered the study with 120 fractures

Age: mean 43 years

Gender: 73 males $(61 \%)$ and 46 females

Fracture type: Closed, unstable fractures. Classified according to the Schatzker system: type I, 1 fracture; type II, 52 fractures; type III, 26 fractures; type IV, 11 fractures; type V, 28 fractures; type VI, 2 fractures

Injury severity: injury severity score not reported; unknown percentage of traffic injuries Inclusion criteria: patient age of 16 to 77 years with an acute, closed, unstable fracture of the proximal part of the tibia (Schatzker types I to VI) that required both internal fixation and grafting

Exclusion criteria: Patients with substantial metabolic bone disease; diabetes, malignancy, peripheral vascular disease, alcoholism, substance abuse, use of systemic steroids, or immunosuppressive therapy; infection at the operative site; concurrent treatment with other bone substitutes including autograft (any graft substance other than the bone graft substitute (alpha-BSM) or autologous iliac bone graft); or related peripheral nerve damage were excluded, as were women who were pregnant or breastfeeding and fertile women not on routine contraceptive control. Tobacco use was not an exclusion criterion

Interventions

Surgical procedure: All fractures underwent ORIF with use of standard non-locking plate-fixation techniques in accordance with the surgeon's normal practice. Plate-andscrew constructs were used in 109 fractures, and screws only were used in 9. After reduction of the articular fracture, the residual subarticular defect was measured and then was packed with either:

Bone filler 1: alpha-BSM ( $\mathrm{n}=82$ fractures)

Bone filler 2: morselised corticocancellous autologous iliac bone graft ( $\mathrm{n}=38$ fractures)

Outcomes

Follow-up: 6, 12 and up to 48 months.

Outcome measures: complications, union, subsidence, loss or premature resorption of the graft, infection, range of knee motion, donor site pain, varus malunion, articular depression

Notes

Randomisation for subarticular defect management with autologous iliac bone graft or alpha-BSM was based on a 1:2 protocol, respectively, as autologous iliac bone graft was considered to be associated with more risks of morbidity to the patient. This randomi- 
sation protocol was mandated by the FDA

\section{Risk of bias}

\begin{tabular}{|c|c|c|}
\hline Bias & Authors' judgement & Support for judgement \\
\hline $\begin{array}{l}\text { Random sequence generation (selection } \\
\text { bias) }\end{array}$ & Low risk & $\begin{array}{l}\text { "Patients were randomised by sealed, computer-generated ran- } \\
\text { domisation schedules" }\end{array}$ \\
\hline Allocation concealment (selection bias) & Low risk & $\begin{array}{l}\text { The envelopes were opened in the operating room to determine } \\
\text { which grafting material would be used }\end{array}$ \\
\hline $\begin{array}{l}\text { Blinding of participants and personnel } \\
\text { (performance bias) } \\
\text { All outcomes }\end{array}$ & High risk & $\begin{array}{l}\text { There was no mention of blinding. Given the contrast in the } \\
\text { interventions, it is unlikely that blinding was done }\end{array}$ \\
\hline $\begin{array}{l}\text { Blinding of outcome assessment (detection } \\
\text { bias) } \\
\text { All outcomes }\end{array}$ & High risk & $\begin{array}{l}\text { All radiographs were reviewed by the panel of } 2 \text { experienced or- } \\
\text { thopaedic trauma surgeons and } 1 \text { consultant, who were blinded } \\
\text { as to the kind of graft material used. Although the panellists were } \\
\text { blinded to treatment group, the trial authors recognised that in- } \\
\text { dividual reviewers may have been able to detect the difference } \\
\text { in treatment, resulting in detection bias }\end{array}$ \\
\hline $\begin{array}{l}\text { Incomplete outcome data (attrition bias) } \\
\text { All outcomes }\end{array}$ & High risk & $\begin{array}{l}\text { There were inconsistencies between text and table for loss to fol- } \\
\text { low-up. The proportion differed in the } 2 \text { groups; e.g. } 12 \text { months } \\
\text { follow-up: } 15 / 82(18.3 \%) \text { versus } 12 / 38 \text { ( } 31.6 \%)\end{array}$ \\
\hline Selective reporting (reporting bias) & Unclear risk & $\begin{array}{l}\text { All outcomes reported as being collected were reported in the } \\
\text { 'Results' section. No reference to a published protocol or trial } \\
\text { registration number was provided and none was found }\end{array}$ \\
\hline Other bias & Unclear risk & $\begin{array}{l}\text { Separate baseline data for the two groups not provided except } \\
\text { for mean age ( } 43 \text { years in both groups) and mean weight ( } 77.6 \\
\mathrm{~kg} \text { versus } 83 \mathrm{~kg} \text { ) }\end{array}$ \\
\hline
\end{tabular}

Shen 2011

Methods

Participants
Single-centre, quasi-randomised controlled trial

Country of study: China

Sample size: 58 participants

Age: mean 36 years, range 20 to 56 years

Gender: 40 males (69\%) and 18 females

Fracture type: all presented with closed Schatzker type II (26 fractures) or type III fractures (32)

Injury severity: injury severity score not reported; 44 (76\%) were traffic injuries Inclusion criteria: fractures operated on within two weeks of injury; Schatzker II or III tibial plateau fractures (type II was considered a splitting lateral platform collapse, lateral 
Shen 2011 (Continued)

buckling stress fracture due to vertical load, and type III was regarded as a collapse on the outside of the plateau and common central area collapse); no blood, urine, liver, renal and heart failure; patient physically capable of undergoing surgery; mild or moderate bone osteoporosis; suitable for internal fixation of the joint line; no serious neurological or vascular damage injury

Exclusion criteria: open fracture; no other joint fracture or dislocation; pathologic fractures including fibrous tumours, giant cell tumour, or bone cyst; peroneal nerve injury, vascular rupture or knee joint capsule rupture more in severe cases; documented mental illness, epilepsy or inability to provide informed consent

Interventions

Surgical procedure 1: Arthroscopically assisted percutaneous internal fixation $(\mathrm{n}=38)$ . Arthroscopic anterolateral portal approach used to remove haematoma and reduce fracture and meniscal repair as indicated. Reduction radiologically aided with C-arm fluoroscopy. Kirschner wire used for temporary reduction. Fracture fixation devices for Schatzker II fractures included cancellous lag screw $(\mathrm{n}=12)$ and anatomical plate fixation $(n=6)$. Implant used not reported in 20 participants aside from being a plate

Surgical procedure 2: Open reduction and internal fixation $(n=20)$ using an anteromedial or anterolateral longitudinal or inverted L-shaped incision. Fracture reduction under direct vision. Kirschner wire temporary fixation and confirmed reduction using C-arm fluoroscopy. Fracture fixation devices used include cancellous lag screws $(\mathrm{n}=11)$ and anatomical plates $(\mathrm{n}=9)$

Postoperative management same between groups. Compression dressing applied and continuous passive motion machine mobilisation commenced during the initial 3 postoperative days. Initial non-weight bearing stipulated. Weight-bearing commenced gradually from 6 weeks postoperatively. No further information provided

Outcomes

Follow-up: Monthly assessment during initial 6 months, then once every 2 months up to 12 to 14 postoperative months

Outcome measures: Hospital Special Surgery (HSS) knee function score, length of surgery, incision length, fracture union and time to radiological evidence of fracture union, complications, early joint pain and range of movement

Notes

Incomplete information provided on fracture fixation methods and devices used in the arthroscopically assisted group

Risk of bias

\begin{tabular}{l|l|l} 
Bias & Authors judgement & Support for judgement \\
\hline $\begin{array}{l}\text { Random sequence generation (selection } \\
\text { bias) }\end{array}$ & High risk & $\begin{array}{l}\text { Randomisation performed using hospital } \\
\text { medical record numbers. Imbalance be- } \\
\text { tween groups not explained }\end{array}$ \\
\hline Allocation concealment (selection bias) & High risk & Quasi-randomised and thus not concealed \\
\hline $\begin{array}{l}\text { Blinding of participants and personnel } \\
\text { (performance bias) } \\
\text { All outcomes }\end{array}$ & High risk & $\begin{array}{l}\text { There was no mention of blinding. Given } \\
\text { the contrast in the interventions, blinding } \\
\text { is not possible for either the personnel or } \\
\text { participants }\end{array}$
\end{tabular}

Surgical fixation methods for tibial plateau fractures (Review)

Copyright () 2015 The Cochrane Collaboration. Published by John Wiley \& Sons, Ltd. 
Shen 2011 (Continued)

\begin{tabular}{|c|c|c|}
\hline $\begin{array}{l}\text { Blinding of outcome assessment (detection } \\
\text { bias) } \\
\text { All outcomes }\end{array}$ & High risk & There was no mention of blinding. \\
\hline $\begin{array}{l}\text { Incomplete outcome data (attrition bias) } \\
\text { All outcomes }\end{array}$ & Low risk & There was no reported loss to follow-up. \\
\hline Selective reporting (reporting bias) & Unclear risk & $\begin{array}{l}\text { All outcomes reported as being collected } \\
\text { were reported in the results section. There } \\
\text { was no reference to a published protocol } \\
\text { or trial registration number, and none was } \\
\text { found }\end{array}$ \\
\hline Other bias & High risk & $\begin{array}{l}\text { The paper indicated limited control regard- } \\
\text { ing fixation methods, which could have af- } \\
\text { fected the results. There was, however, 'no } \\
\text { significant difference' reported in baseline } \\
\text { characteristics in terms of gender, age, frac- } \\
\text { ture type and time to injury }\end{array}$ \\
\hline
\end{tabular}

\section{$\nabla \times$}

ACL: anterior cruciate ligament; AO: Arbeitsgemeinschaft für Osteosynthesefragen (German for 'Association for the Study of Internal Fixation'); FDA: Food and Drug Administration (USA); KOOS: knee injury and osteoarthritis outcome score; ORIF: open reduction and internal fixation; OTA: Orthopaedic Trauma Association; RCT: randomised controlled trial.

\section{Characteristics of excluded studies [ordered by study ID]}

\begin{tabular}{|c|c|}
\hline Study & Reason for exclusion \\
\hline Carter 1996 & Excluded as only published as an abstract. Not an RCT. \\
\hline Chan 2012 & Not an RCT. Retrospective cohort study. \\
\hline Dall'oca 2012 & $\begin{array}{l}\text { Not an RCT. A comparison of arthroscopic fixation of fractures with open reduction internal fixation. The study } \\
\text { was a comparison of results of two different cohorts of patients }\end{array}$ \\
\hline Egol 2004b & $\begin{array}{l}\text { A report on a cohort of patients treated by LISS technique and then a biomechanical study of the LISS plate } \\
\text { versus the double plating technique in cadaveric tibiae }\end{array}$ \\
\hline Gunther 2008 & Not a comparison of two fracture fixation methods. \\
\hline Hansen 2009 & Not a randomised controlled trial. Not a comparison of fixation of tibial plateau fractures \\
\hline Jubel 2004 & Not an RCT. A cohort study of synthetic bone graft substitute \\
\hline
\end{tabular}


(Continued)

Krupp $2009 \quad$ Not an RCT. A comparison of outcomes of a cohort of patients treated by either open reduction and internal fixation versus fine wire fixator

Lobenhoffer 1999 Not an RCT. A report on outcomes after fixation of tibial plateau fractures

Malakasi 2013 Not an RCT. A comparison of outcomes of a cohort of patients treated by either open reduction and internal fixation versus hybrid external fixator

NCT00429585 Not the population of interest. Population consists of extracapsular proximal tibial fractures without intraarticular extension

NCT00610701 This RCT aimed to compare the differences between anteriorly placed femoral external fixator pins and laterally placed femoral external fixator pins for people following tibial plateau fracture. It was registered in January 2008 but terminated (the trial registration record was updated in September 2014) because of "extremely low recruitment and very little follow-up for meaningful data"

Ohdera $2003 \quad$ Not an RCT. A retrospective cohort study

Ong $2012 \quad$ Not an RCT. A comparative cohort study.

Sung 2008 Not comparison of two fracture fixation methods. The trial compared reused components of an external fixator versus new external fixator components

Tahririan $2014 \quad$ Not an RCT. A prospective comparison of locking versus non-locking plate fixation

Weaver 2012 Not an RCT. Study evaluated the risk of loss of fixation with the injury severity pattern of the fracture

Zhang 2012 Not an RCT. A retrospective analysis of the outcomes of treating bicondylar tibial plateau fractures with a dual plating technique

Zhuo 2008

Not an RCT. A study examining the effect of a synthetic bone graft substitute in the healing of different fractures around the body

LISS: less invasive stabilisation system; RCT: randomised controlled trial.

\section{Characteristics of studies awaiting assessment [ordered by study ID] \\ Jordan 2014}

Methods Single-centre RCT (computer-generated sequence)

Participants Sample size: 24

Inclusion criteria: depressed or split depressed tibial plateau fracture (medial or lateral) requiring surgical intervention, age 18 to 80 , informed consent

Exclusion criteria: concomitant injuries influencing the management of the tibial plateau fracture, contraindication 
Outcomes Follow-up: 3, 6 and 12 months

Primary outcome measure: quality of reduction based on the postoperative CT scan

Secondary outcome measures: any surgical complication and patient satisfaction, measured using the Oxford Knee Score and Short Form-12 (SF12) questionnaire at 3, 6 and 12 months

Notes

Published protocol: Jordan R, Hao J, Fader R, Gibula D, Mauffrey C. Study protocol: Trial of inflation osteoplasty in the management of tibial plateau fractures. European Journal of Orthopaedic Surgery and Traumatology 2014;24 (5):647-53

Current status: not known

The eligibility of this ongoing study will be resolved in the next version of this review

\section{Characteristics of ongoing studies [ordered by study ID]}

\section{Kuros Biosurgery AG}

\begin{tabular}{|c|c|}
\hline Trial name or title & $\begin{array}{l}\text { Phase } 2 \text {, randomised controlled, open-label (dose-blinded), multi-centre, dose-finding study of the safety and } \\
\text { efficacy of I-0401 in the treatment of patients with fractures of the tibial plateau requiring grafting }\end{array}$ \\
\hline Methods & A phase 2 randomised, controlled, open-label (dose-blinded), multi-centre, dose-finding study \\
\hline Participants & $\begin{array}{l}\text { Sample size: } 183 \text { (see Notes) } \\
\text { Inclusion criteria: radiological evidence of fracture of the tibial plateau requiring grafting and fixation that } \\
\text { occurs either alone or is part of a polytraumatic event (AO classification: } 41 \mathrm{~B} 2,41 \mathrm{~B} 3,41 \mathrm{C} 2,41 \mathrm{C} 3 \text { ); female } \\
\text { and male subjects aged } 18 \text { years or above; body mass index (BMI) 16-33 (minimum body weight } 50 \mathrm{~kg} \text {, } \\
\text { maximum } 140 \mathrm{~kg} \text { ); Glasgow coma score (GCS) = 13; females of child-bearing potential must be willing to } \\
\text { undergo a pregnancy test (urine) prior to treatment start (at screening); females of child-bearing potential } \\
\text { randomised in the intervention group must agree to have acceptable contraception for at least } 3 \text { months after } \\
\text { receiving I-0401; able and willing to come to the clinic for follow-up visits as scheduled in the time and events } \\
\text { schedule; signed informed consent form. The patient has to be able to give consent personally } \\
\text { Exclusion criteria: total size of defect requiring a graft volume of > } 7.0 \mathrm{~mL} \text {; patients with high risk of } \\
\text { amputation; open tibial plateau fractures Gustilo-Anderson grade III; concomitant ipsilateral fractures of } \\
\text { the limb other than the fracture of the tibial plateau; active or past history of malignant tumour; evidence } \\
\text { of systemic or localised infection at time of surgery; pregnant or lactating females; evidence of immune- } \\
\text { suppression; on treatment or planned treatment with products containing PTH (e.g. Forteo, Forsteo); evidence } \\
\text { of hypercalcaemia (serum calcium above ULN); known history of allergy to aesthetics; suspected or known } \\
\text { allergies towards any of the components of I-0401 (TGplPTH1-34, Fibrin, HA/TCP granules); history or } \\
\text { evidence for a metabolic bone disease other than primary osteoporosis; known clinically significant organ or } \\
\text { systemic diseases or any other relevant medical condition deemed by the investigator to impose hazard to the } \\
\text { patient if study therapy is initiated or to compromise the subject's participation in the study; participation in } \\
\text { another clinical trial within } 3 \text { months prior to trial start; evidence of moderate or severe renal failure (serum } \\
\text { creatinine > } 3.0 \text { times ULN, NCI-CTC grades } 3 \text { and } 4 \text { ); history of allergic thrombocytopaenia (type II) } \\
\text { induced by heparin; inexplicable elevations of alkaline phosphatase (alkaline phosphatase > 5.0 times ULN, }\end{array}$ \\
\hline
\end{tabular}




\section{Kuros Biosurgery AG (Continued)}

NCI-CTC grades 3 and 4); prior external beam or implant radiation therapy to the skeleton

Group 1: dose $1(0.4 \mathrm{mg} / \mathrm{ml})$ : drug: I-0401 (a bone graft substitute in fibrin supplemented with hydroxyap-
atite/beta tricalcium phosphate granules) (also KUR-111)
Group 2: dose $2(1.0 \mathrm{mg} / \mathrm{ml})$ : drug: I-0401 (a bone graft substitute in fibrin supplemented with hydroxyap-
atite/beta tricalcium phosphate granules) (also KUR-111)
Group 3: autograft

Outcomes

Follow-up: 6, 12, 16, 26, 52 and 104 weeks postoperatively

Primary outcome measure(s):

- Radiological fracture union after implantation of the graft. Assessments will be performed by an independent radiological panel and will be based on computed tomography. Radiological fracture union will be defined using the following criteria.

- Cortical bridging on at least 1 visible cortical plane.

- Obliteration of fracture lines.

- Dislocation of bone fragments compared to the postoperative film.

- The tolerability and safety of I-0401.

- Pharmacokinetics of the drug.

- Quality of life and pharmacoeconomics.

Secondary outcome measures:

- Loss of reduction of the tibial plateau (in $\mathrm{mm}$ ) at after surgery compared to the postoperative assessment as measured by an independent radiological panel.

- Proportion of patients with radiological fracture union as assessed by an independent radiological panel.

- Proportion of patients with fracture healing at 16 weeks after implantation of the graft as assessed by the investigators, considering clinical and radiological criteria. Clinical assessment will consider the level of pain upon weight bearing on fracture site, the level of redness/swelling of the knee and whether a secondary intervention was necessary to promote fracture healing. Radiological criteria will consider cortical bridging and fracture lines.

- Time to fracture healing as assessed by the investigators considering clinical and radiological criteria (see above for fracture healing assessment).

- The number of secondary interventions due to non-healing within 52 weeks and within 104 weeks from time of surgery comprises all surgical procedures to promote fracture-healing; all surgical procedures to perform a knee arthroplasty; and non-invasive treatments (e.g. ultrasound) to promote fracture-healing. Timepoint(s) of evaluation of this end point: (i) loss of reduction of the tibia plateau (in $\mathrm{mm}$ ) at $6,12,16$, 26, 52 and 104 weeks after surgery; (ii) the proportion of patients with radiological fracture union at 52 and 104 weeks; (iii) proportion of patients with fracture healing at 16 weeks after implantation of the graft as assessed by the investigators considering clinical and radiological criteria.

- Time to fracture healing as assessed by the investigators.

- The number of secondary interventions due to non-healing within 52 weeks and within 104 weeks from time of surgery comprises all surgical procedures to promote fracture-healing; all surgical procedures to perform a knee arthroplasty; and non-invasive treatments (e.g. ultrasound) to promote fracture-healing.

Starting date $\quad 27$ February 2007

Contact information Chief Medical Officer, Kuros Biosurgery AG, Technoparkstrasse 1, 8005, Zürich, Switzerland. Email: info@kuros.ch 
Trial name or title Cerament treatment of fracture defects (CERTiFy)

\begin{tabular}{|c|c|}
\hline Methods & Open label RCT \\
\hline Participants & $\begin{array}{l}\text { Sample size: } 136 \\
\text { Inclusion criteria: patients with traumatic, closed, depression fracture of the proximal tibia (limited to AO } \\
\text { type B2 \& B3); solitary trauma; candidate for bone grafting; aged between } 18 \text { and } 65 \text { years; written informed } \\
\text { consent obtained before any study-related activities; patients with communicative ability to understand the } \\
\text { procedure and participate in the study and comply with the follow-up programme } \\
\text { Exclusion criteria: patients with multiple injuries; polytrauma patients; compartment syndrome; previous } \\
\text { iliac crest bone graft harvesting; local infection at the site of implantation; chronic pain disease; malignancy; } \\
\text { rheumatoid arthritis; chronic cortisone intake; radiographic diagnostics not available, fracture cannot be } \\
\text { classified; clinically significant or unstable medical or surgical condition that may preclude safe and complete } \\
\text { study participation; a pre-existing calcium metabolism disorder (e.g. hypercalcaemia); known hyperthyroidism } \\
\text { or autonomous thyroid adenoma; history of serious reaction to iodine-based radio contrast agents; women } \\
\text { who are pregnant or breastfeeding; irreversible coagulopathy or bleeding disorder; history of physical or } \\
\text { psychological condition that contraindicates the use of an investigational device or render the patient at } \\
\text { high risk from treatment complications; history of hypersensitivity to the investigational device or any of its } \\
\text { ingredients; participation in other clinical trials during the present clinical trial or within the last month }\end{array}$ \\
\hline Interventions & $\begin{array}{l}\text { Group 1: bone graft substitute - CERAMENTTM/BONE VOID FILLER } \\
\text { Group 2: bone graft - autologous cancellous bone graft (iliac crest) }\end{array}$ \\
\hline Outcomes & $\begin{array}{l}\text { Follow-up: } 26 \text { weeks postoperatively } \\
\text { Primary outcome measures: Short Form-12 Physical Component Summary (PCS); global pain visual ana- } \\
\text { logue scale score } \\
\text { Secondary outcome measures: utilisation of costs of care-related resources; SF-12 Mental Component } \\
\text { Summary (MCS); bone healing; occurrence of adverse events; device complaints and device-related incidents; } \\
\text { frequencies of subjects experiencing at least one adverse event (AE) will be displayed by body system and } \\
\text { preferred term according to MedDRA terminology. Detailed information collected for each AE will include } \\
\text { a description of the event, duration, whether the AE was serious, intensity, relationship to trial device, action } \\
\text { taken and clinical outcome }\end{array}$ \\
\hline
\end{tabular}




\section{NCT01828905 (Continued)}

Contact information Pol M. Rommens, Univ.-Prof, Department of Trauma Surgery, Centre for Musculoskeletal Surgery, Johannes Gutenberg University Mainz, Mainz, Rhineland-Palatine, Germany. Email: pol.rommens@unimedizinmainz.de

Notes

ClinicalTrials.gov identifier: NCT01828905

Published protocol: Nusselt T, Hofmann A, Wachtlin D, Gorbulev S, Rommens PM. CERAMENT treatment of fracture defects (CERTiFy): protocol for a prospective, multicenter, randomised study investigating the use of CERAMENTTM BONE VOID FILLER in tibial plateau fractures. Trials. 2014 8;15:75

Current status: Recruiting. Last updated: 30 March 2015. Estimated study completion date: December 2016

NCT02035917

Trial name or title Comparison of tibial plateau fractures outcomes treated with non-locking and locking plate

Methods It is not clear whether this is a randomised controlled trial rather than a prospective comparison as in their previous study (Tahririan 2014).

Participants Sample size: not stated

Inclusion criteria: unilateral closed tibia plateau fracture, aged 19 to 60 years

Exclusion criteria: patients who have been treated with casting or screw, history of diabetes mellitus, pathological fractures

Interventions $\quad$ Group 1: locking plate for open reduction internal fixation of a tibial plateau fracture

Group 2: non-locking plate for open reduction internal fixation of a tibial plateau fracture

Outcomes $\quad$ Follow-up: 10 months postoperatively

Primary outcome measures: Knee Society knee score

Secondary outcome measures: none stated

Starting date Not stated

Contact information Amin Nemati, Orthopedic Surgery Resident. Department of Orthopedics, Kashani Hospital, Isfahan University of Medical Sciences, Isfahan, Iran. Email: nemati@med.mui.ac.ir

Notes

ClinicalTrials.gov identifier: NCT02035917

Estimated primary completion date: April 2014

Last verified: 11th January 2014. Current status: Recruiting.

AO: Arbeitsgemeinschaft für Osteosynthesefragen (German for 'Association for the Study of Internal Fixation'); MedDRA: Medical Dictionary for Regulatory Activities; NCI-CTC: National Cancer Institute's Common Terminology Criteria [for adverse events]; PTH: parathyroid hormone; RCT: randomised controlled trial; TCP/HA: Tricalcium phosphate/hydroxyapatite; ULN: upper limit of normal; 
DATA AND ANALYSES

\begin{abstract}
Comparison 1. Circular external fixation with limited internal fixation (hybrid fixation) versus standard open reduction internal fixation
\end{abstract}

\begin{tabular}{|c|c|c|c|c|}
\hline Outcome or subgroup title & $\begin{array}{l}\text { No. of } \\
\text { studies }\end{array}$ & $\begin{array}{c}\text { No. of } \\
\text { participants }\end{array}$ & Statistical & Effect size \\
\hline 1 SF-36 scores at 24 months & 1 & & Mean Difference (IV, Random, 95\% CI) & Totals not selected \\
\hline 1.1 General health & 1 & & Mean Difference (IV, Random, 95\% CI) & $0.0[0.0,0.0]$ \\
\hline 1.2 Physical function & 1 & & Mean Difference (IV, Random, 95\% CI) & $0.0[0.0,0.0]$ \\
\hline 1.3 Role-physical & 1 & & Mean Difference (IV, Random, 95\% CI) & $0.0[0.0,0.0]$ \\
\hline 1.4 Role-emotional & 1 & & Mean Difference (IV, Random, 95\% CI) & $0.0[0.0,0.0]$ \\
\hline 1.5 Social function & 1 & & Mean Difference (IV, Random, 95\% CI) & $0.0[0.0,0.0]$ \\
\hline 1.6 Bodily pain & 1 & & Mean Difference (IV, Random, 95\% CI) & $0.0[0.0,0.0]$ \\
\hline 1.7 Vitality & 1 & & Mean Difference (IV, Random, 95\% CI) & $0.0[0.0,0.0]$ \\
\hline 1.8 Mental health & 1 & & Mean Difference (IV, Random, 95\% CI) & $0.0[0.0,0.0]$ \\
\hline 2 Hospital for Special Surgery & 1 & & Mean Difference (IV, Fixed, 95\% CI) & Totals not selected \\
\hline \multicolumn{5}{|l|}{ Score } \\
\hline 2.1 At 6 months & 1 & & Mean Difference (IV, Fixed, 95\% CI) & $0.0[0.0,0.0]$ \\
\hline 2.2 At 12 months & 1 & & Mean Difference (IV, Fixed, 95\% CI) & $0.0[0.0,0.0]$ \\
\hline 2.3 At 24 months & 1 & & Mean Difference (IV, Fixed, 95\% CI) & $0.0[0.0,0.0]$ \\
\hline 3 WOMAC Pain score & 1 & & Mean Difference (IV, Fixed, 95\% CI) & Totals not selected \\
\hline 3.1 At 6 months & 1 & & Mean Difference (IV, Fixed, 95\% CI) & $0.0[0.0,0.0]$ \\
\hline 3.2 At 12 months & 1 & & Mean Difference (IV, Fixed, 95\% CI) & $0.0[0.0,0.0]$ \\
\hline 3.3 At 24 months & 1 & & Mean Difference (IV, Fixed, 95\% CI) & $0.0[0.0,0.0]$ \\
\hline 4 WOMAC Stiffness score & 1 & & Mean Difference (IV, Fixed, 95\% CI) & Totals not selected \\
\hline 4.1 At 6 months & 1 & & Mean Difference (IV, Fixed, 95\% CI) & $0.0[0.0,0.0]$ \\
\hline 4.2 At 12 months & 1 & & Mean Difference (IV, Fixed, 95\% CI) & $0.0[0.0,0.0]$ \\
\hline 4.3 At 24 months & 1 & & Mean Difference (IV, Fixed, 95\% CI) & $0.0[0.0,0.0]$ \\
\hline 5 WOMAC Function score & 1 & & Mean Difference (IV, Fixed, 95\% CI) & Totals not selected \\
\hline 5.1 At 6 months & 1 & & Mean Difference (IV, Fixed, 95\% CI) & $0.0[0.0,0.0]$ \\
\hline 5.2 At 12 months & 1 & & Mean Difference (IV, Fixed, 95\% CI) & $0.0[0.0,0.0]$ \\
\hline 5.3 At 24 months & 1 & & Mean Difference (IV, Fixed, 95\% CI) & $0.0[0.0,0.0]$ \\
\hline 6 Reoperations (24 months) & 1 & & Risk Ratio (M-H, Fixed, 95\% CI) & Totals not selected \\
\hline 6.1 Requirement for & 1 & & Risk Ratio (M-H, Fixed, 95\% CI) & $0.0[0.0,0.0]$ \\
\hline \multicolumn{5}{|l|}{ reoperation } \\
\hline $\begin{array}{l}6.2 \text { Reoperation: incision and } \\
\text { drainage }\end{array}$ & 1 & & Risk Ratio (M-H, Fixed, 95\% CI) & $0.0[0.0,0.0]$ \\
\hline $\begin{array}{l}\text { 6.3 Reoperation: } \\
\text { split-thickness skin graft }\end{array}$ & 1 & & Risk Ratio (M-H, Fixed, 95\% CI) & $0.0[0.0,0.0]$ \\
\hline $\begin{array}{l}6.4 \text { Reoperation: screw } \\
\text { removal }\end{array}$ & 1 & & Risk Ratio (M-H, Fixed, 95\% CI) & $0.0[0.0,0.0]$ \\
\hline $\begin{array}{l}6.5 \text { Reoperation: knee } \\
\text { manipulation }\end{array}$ & 1 & & Risk Ratio (M-H, Fixed, 95\% CI) & $0.0[0.0,0.0]$ \\
\hline $\begin{array}{l}\text { 6.6 Reoperation: plate } \\
\text { removal }\end{array}$ & 1 & & Risk Ratio (M-H, Fixed, 95\% CI) & $0.0[0.0,0.0]$ \\
\hline $\begin{array}{l}6.7 \text { Reoperation: total knee } \\
\text { arthroplasty }\end{array}$ & 1 & & Risk Ratio (M-H, Fixed, 95\% CI) & $0.0[0.0,0.0]$ \\
\hline
\end{tabular}

Surgical fixation methods for tibial plateau fractures (Review)

Copyright @ 2015 The Cochrane Collaboration. Published by John Wiley \& Sons, Ltd. 


\begin{tabular}{|c|c|c|c|}
\hline $\begin{array}{l}\text { 6.8 Reoperation: above knee } \\
\text { amputation }\end{array}$ & 1 & Risk Ratio (M-H, Fixed, 95\% CI) & $0.0[0.0,0.0]$ \\
\hline $\begin{array}{l}\text { 6.9 Reoperation: soft-tissue } \\
\text { flap }\end{array}$ & 1 & Risk Ratio (M-H, Fixed, 95\% CI) & $0.0[0.0,0.0]$ \\
\hline $\begin{array}{l}\text { 6.10 Reoperation: revision } \\
\text { ORIF }\end{array}$ & 1 & Risk Ratio (M-H, Fixed, 95\% CI) & $0.0[0.0,0.0]$ \\
\hline $\begin{array}{l}7 \text { Intra-operative measures (length } \\
\text { of surgery, blood loss) }\end{array}$ & 1 & Mean Difference (IV, Fixed, 95\% CI) & Totals not selected \\
\hline 7.1 Length of surgery (min) & 1 & Mean Difference (IV, Fixed, 95\% CI) & $0.0[0.0,0.0]$ \\
\hline 7.2 Blood loss (ml) & 1 & Mean Difference (IV, Fixed, 95\% CI) & $0.0[0.0,0.0]$ \\
\hline $\begin{array}{l}8 \text { Knee joint total range of motion } \\
\text { (degrees) at } 24 \text { months }\end{array}$ & 1 & Mean Difference (IV, Fixed, 95\% CI) & Totals not selected \\
\hline $\begin{array}{l}9 \text { Return to pre-injury activity } \\
\text { level }\end{array}$ & 1 & Risk Ratio (M-H, Fixed, 95\% CI) & Totals not selected \\
\hline 9.1 At 6 months & 1 & Risk Ratio (M-H, Fixed, 95\% CI) & $0.0[0.0,0.0]$ \\
\hline 9.2 At 12 months & 1 & Risk Ratio (M-H, Fixed, 95\% CI) & $0.0[0.0,0.0]$ \\
\hline 9.3 At 24 months & 1 & Risk Ratio (M-H, Fixed, 95\% CI) & $0.0[0.0,0.0]$ \\
\hline 10 Length of hospital stay (days) & 1 & Mean Difference (IV, Fixed, 95\% CI) & Totals not selected \\
\hline $\begin{array}{l}11 \text { Radiological outcomes } \\
\text { (postoperatively) }\end{array}$ & 1 & Risk Ratio (M-H, Fixed, 95\% CI) & Totals not selected \\
\hline $\begin{array}{l}\text { 11.1 Lateral plateau step }>5 \\
\mathrm{~mm}\end{array}$ & 1 & Risk Ratio (M-H, Fixed, 95\% CI) & $0.0[0.0,0.0]$ \\
\hline $\begin{array}{l}11.2 \text { Medial plateau step }>5 \\
\mathrm{~mm}\end{array}$ & 1 & Risk Ratio (M-H, Fixed, 95\% CI) & $0.0[0.0,0.0]$ \\
\hline $\begin{array}{l}11.3 \text { Medial plateau } \\
\text { depression }>5 \mathrm{~mm}\end{array}$ & 1 & (M-H, Fixed, 95\% CI) & $0.0[0.0,0.0]$ \\
\hline $\begin{array}{l}11.4 \text { Lateral plateau } \\
\text { depression }>5 \mathrm{~mm}\end{array}$ & 1 & Risk Ratio (M-H, Fixed, 95\% CI) & $0.0[0.0,0.0]$ \\
\hline $\begin{array}{l}11.5 \text { Fracture gap deformity > } \\
5 \mathrm{~mm}\end{array}$ & 1 & Risk Ratio (M-H, Fixed, 95\% CI) & $0.0[0.0,0.0]$ \\
\hline $\begin{array}{l}\text { 11.6 Metaphyseal-diaphyseal } \\
\text { angulation }>5 \text { degrees }\end{array}$ & & Risk Ratio (M-H, Fixed, 95\% CI) & $0.0[0.0,0.0]$ \\
\hline $\begin{array}{l}12 \text { Radiological evidence of } \\
\text { tibiofemoral joint osteoarth }\end{array}$ & 1 & Risk Ratio (M-H, Fixed, 95\% CI) & Totals not selected \\
\hline 12.1 At 12 months & 1 & Risk Ratio (M-H, Fixed, 95\% CI) & $0.0[0.0,0.0]$ \\
\hline 12.2 At 24 months & 1 & Risk Ratio (M-H, Fixed, 95\% CI) & $0.0[0.0,0.0]$ \\
\hline
\end{tabular}

Comparison 2. Less invasive stabilisation system (LISS) versus double-plating open reduction internal fixation

\begin{tabular}{|c|c|c|c|c|}
\hline Outcome or subgroup title & $\begin{array}{l}\text { No. of } \\
\text { studies }\end{array}$ & $\begin{array}{c}\text { No. of } \\
\text { participants }\end{array}$ & Statistical method & Effect size \\
\hline $\begin{array}{l}1 \text { Hospital for Special Surgery } \\
\text { Score }\end{array}$ & 1 & & Mean Difference (IV, Fixed, 95\% CI) & Totals not selected \\
\hline $\begin{array}{l}1.1 \text { Hospital for Special } \\
\text { Surgery (12 months) }\end{array}$ & 1 & & Mean Difference (IV, Fixed, 95\% CI) & $0.0[0.0,0.0]$ \\
\hline $\begin{array}{l}1.2 \text { Hospital for Special } \\
\text { Surgery Score ( } 24 \text { months) }\end{array}$ & 1 & & Mean Difference (IV, Fixed, 95\% CI) & $0.0[0.0,0.0]$ \\
\hline 2 Complications (24 months) & 1 & & Risk Ratio (M-H, Fixed, 95\% CI) & Totals not selected \\
\hline
\end{tabular}

Surgical fixation methods for tibial plateau fractures (Review)

Copyright $\odot 2015$ The Cochrane Collaboration. Published by John Wiley \& Sons, Ltd. 
2.1 Seroma

2.2 Haematoma

2.3 Superfical skin infection

2.4 Deep infection

2.5 Deep venous thrombosis

2.6 Hardware failure

2.7 Hardware irritation

2.8 Fracture nonunion

2.9 Loss of fracture reduction

2.10 Loss of alignment

2.11 Total complications

3 Reoperations (up to 24 months)

3.1 Metalwork removal

3.2 Revision fixation

4 Length of surgery ( $\mathrm{min}$ )

5 Incision length $(\mathrm{cm})$

6 Perioperative blood loss (ml)

7 Postsurgical malreduction and malalignment

7.1 Incidence of malreduction

7.2 Incidence of malalignment

8 Time to radiological union

(weeks)
Risk Ratio (M-H, Fixed, 95\% CI)

Risk Ratio (M-H, Fixed, 95\% CI)

Risk Ratio (M-H, Fixed, 95\% CI)

Risk Ratio (M-H, Fixed, 95\% CI)

Risk Ratio (M-H, Fixed, 95\% CI)

Risk Ratio (M-H, Fixed, 95\% CI)

Risk Ratio (M-H, Fixed, 95\% CI)

Risk Ratio (M-H, Fixed, 95\% CI)

Risk Ratio (M-H, Fixed, 95\% CI)

Risk Ratio (M-H, Fixed, 95\% CI)

Risk Ratio (M-H, Fixed, 95\% CI)

Risk Ratio (M-H, Fixed, 95\% CI)

Risk Ratio (M-H, Fixed, 95\% CI)

Risk Ratio (M-H, Fixed, 95\% CI)

Mean Difference (IV, Fixed, 95\% CI)

Mean Difference (IV, Fixed, 95\% CI)

Mean Difference (IV, Fixed, 95\% CI)

Risk Ratio (M-H, Fixed, 95\% CI)

Risk Ratio (M-H, Fixed, 95\% CI)

Risk Ratio (M-H, Fixed, 95\% CI)

Mean Difference (IV, Fixed, 95\% CI)
$0.0[0.0,0.0]$

$0.0[0.0,0.0]$

$0.0[0.0,0.0]$

$0.0[0.0,0.0]$

$0.0[0.0,0.0]$

$0.0[0.0,0.0]$

$0.0[0.0,0.0]$

$0.0[0.0,0.0]$

$0.0[0.0,0.0]$

$0.0[0.0,0.0]$

$0.0[0.0,0.0]$

Totals not selected

$0.0[0.0,0.0]$

$0.0[0.0,0.0]$

Totals not selected

Totals not selected

Totals not selected

Totals not selected

$0.0[0.0,0.0]$

$0.0[0.0,0.0]$

Totals not selected

Comparison 3. Arthroscopically assisted percutaneous reduction and internal fixation versus standard open reduction and internal fixation

\begin{tabular}{|c|c|c|c|c|}
\hline Outcome or subgroup title & $\begin{array}{l}\text { No. of } \\
\text { studies }\end{array}$ & $\begin{array}{c}\text { No. of } \\
\text { participants }\end{array}$ & Statistical method & Effect size \\
\hline $\begin{array}{l}1 \text { Hospital for Special Surgery } \\
\text { Score (14 months) }\end{array}$ & 1 & & Mean Difference (IV, Fixed, 95\% CI) & Totals not selected \\
\hline 2 Knee stiffness & 1 & & Risk Ratio (M-H, Fixed, 95\% CI) & Totals not selected \\
\hline 3 Length of surgery (min) & 1 & & Mean Difference (IV, Fixed, 95\% CI) & Totals not selected \\
\hline 4 Incision length $(\mathrm{cm})$ & 1 & & Mean Difference (IV, Fixed, 95\% CI) & Totals not selected \\
\hline $\begin{array}{l}5 \text { Knee joint range of motion } \\
\text { (degrees) ( } 14 \text { months })\end{array}$ & 1 & & Mean Difference (IV, Fixed, 95\% CI) & Totals not selected \\
\hline $\begin{array}{l}6 \text { Time to radiological union } \\
\text { (months) }\end{array}$ & 1 & & Mean Difference (IV, Fixed, 95\% CI) & Totals not selected \\
\hline
\end{tabular}




\begin{tabular}{|c|c|c|c|c|}
\hline Outcome or subgroup title & $\begin{array}{l}\text { No. of } \\
\text { studies }\end{array}$ & $\begin{array}{c}\text { No. of } \\
\text { participants }\end{array}$ & Statistical method & Effect size \\
\hline 1 Lower limb function & 1 & & Risk Ratio (M-H, Fixed, 95\% CI) & Totals not selected \\
\hline $\begin{array}{l}1.1 \text { Ability to walk (excellent } \\
\text { or good) }\end{array}$ & 1 & & Risk Ratio (M-H, Fixed, 95 & $0.0[0.0,0.0]$ \\
\hline $\begin{array}{l}\text { 1.2 Stair climbing (excellent } \\
\text { or good) }\end{array}$ & 1 & & Risk Ratio (M-H, Fixed, 9 & $0.0[0.0,0.0]$ \\
\hline $\begin{array}{l}\text { 1.3 Squatting (excellent or } \\
\text { good) }\end{array}$ & 1 & & Risk Ratio (M-H, Fixed, 95\% CI) & $0.0[0.0,0.0]$ \\
\hline $\begin{array}{l}1.4 \text { Jumping (excellent or } \\
\text { good) }\end{array}$ & 1 & & Risk Ratio (M-H, Fixed, 95\% CI) & $0.0[0.0,0.0]$ \\
\hline 2 Adverse events & 3 & & Risk Ratio (M-H, Fixed, 95\% CI) & Subtotals only \\
\hline 2.1 Blood transfusion & 1 & 25 & Risk Ratio (M-H, Fixed, 95\% CI) & $0.39[0.04,3.79]$ \\
\hline 2.2 Infection & 3 & 185 & Risk Ratio (M-H, Fixed, 95\% CI) & $1.01[0.27,3.82]$ \\
\hline 2.3 Nonunion & 1 & 120 & Risk Ratio (M-H, Fixed, 95\% CI) & $0.0[0.0,0.0]$ \\
\hline $\begin{array}{l}2.4 \text { Implant breakage or } \\
\text { deformity }\end{array}$ & 1 & 120 & Risk Ratio (M-H, Fixed, 95\% CI) & $0.0[0.0,0.0]$ \\
\hline 2.5 Prominent metalwork & 1 & 40 & Risk Ratio (M-H, Fixed, 95\% CI) & $1.0[0.23,4.37]$ \\
\hline 2.6 Loose screws & 1 & 40 & Risk Ratio (M-H, Fixed, 95\% CI) & $0.33[0.04,2.94]$ \\
\hline $\begin{array}{l}2.7 \text { Donor site complication } \\
\text { (infection) }\end{array}$ & 2 & 160 & Risk Ratio (M-H, Fixed, 95\% CI) & $0.0[0.0,0.0]$ \\
\hline $\begin{array}{l}2.8 \text { Reoperation (for } \\
\text { complication) }\end{array}$ & 1 & & Risk Ratio (M-H, Fixed, 95\% CI) & $1.0[0.16,6.42]$ \\
\hline $\begin{array}{l}3 \text { Range of movement (at } 12 \\
\text { months) }\end{array}$ & 1 & & Risk Ratio (M-H, Fixed, 95\% CI) & Totals not selected \\
\hline 3.1 Flexion $\geq 120$ degrees & 1 & & Risk Ratio (M-H, Fixed, 95\% CI) & $0.0[0.0,0.0]$ \\
\hline 3.2 Full extension & 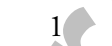 & & Risk Ratio (M-H, Fixed, 95\% CI) & $0.0[0.0,0.0]$ \\
\hline 4 Radiographic outcomes & & & Risk Ratio (M-H, Fixed, 95\% CI) & Totals not selected \\
\hline 4.1 Varus malunion & 1 & & Risk Ratio (M-H, Fixed, 95\% CI) & $0.0[0.0,0.0]$ \\
\hline 4.2 Loss of reduction & 1 & & Risk Ratio (M-H, Fixed, 95\% CI) & $0.0[0.0,0.0]$ \\
\hline $\begin{array}{l}4.3 \text { Articular subsidence } \\
\mathrm{mm}\end{array}$ & 1 & & Risk Ratio (M-H, Fixed, 95\% CI) & $0.0[0.0,0.0]$ \\
\hline $\begin{array}{l}\text { 4.4 Osteoarthritis at } 11 \text { years } \\
\text { follow-up }\end{array}$ & 1 & & Risk Ratio (M-H, Fixed, 95\% CI) & $0.0[0.0,0.0]$ \\
\hline 5 Anatomical malalignment & 1 & & Mean Difference (IV, Fixed, 95\% CI) & Totals not selected \\
\hline $\begin{array}{l}5.1 \text { Valgus malalignment } \\
\text { (degrees) }\end{array}$ & 1 & & Mean Difference (IV, Fixed, 95\% CI) & $0.0[0.0,0.0]$ \\
\hline
\end{tabular}


Analysis I.I. Comparison I Circular external fixation with limited internal fixation (hybrid fixation) versus standard open reduction internal fixation, Outcome I SF-36 scores at 24 months.

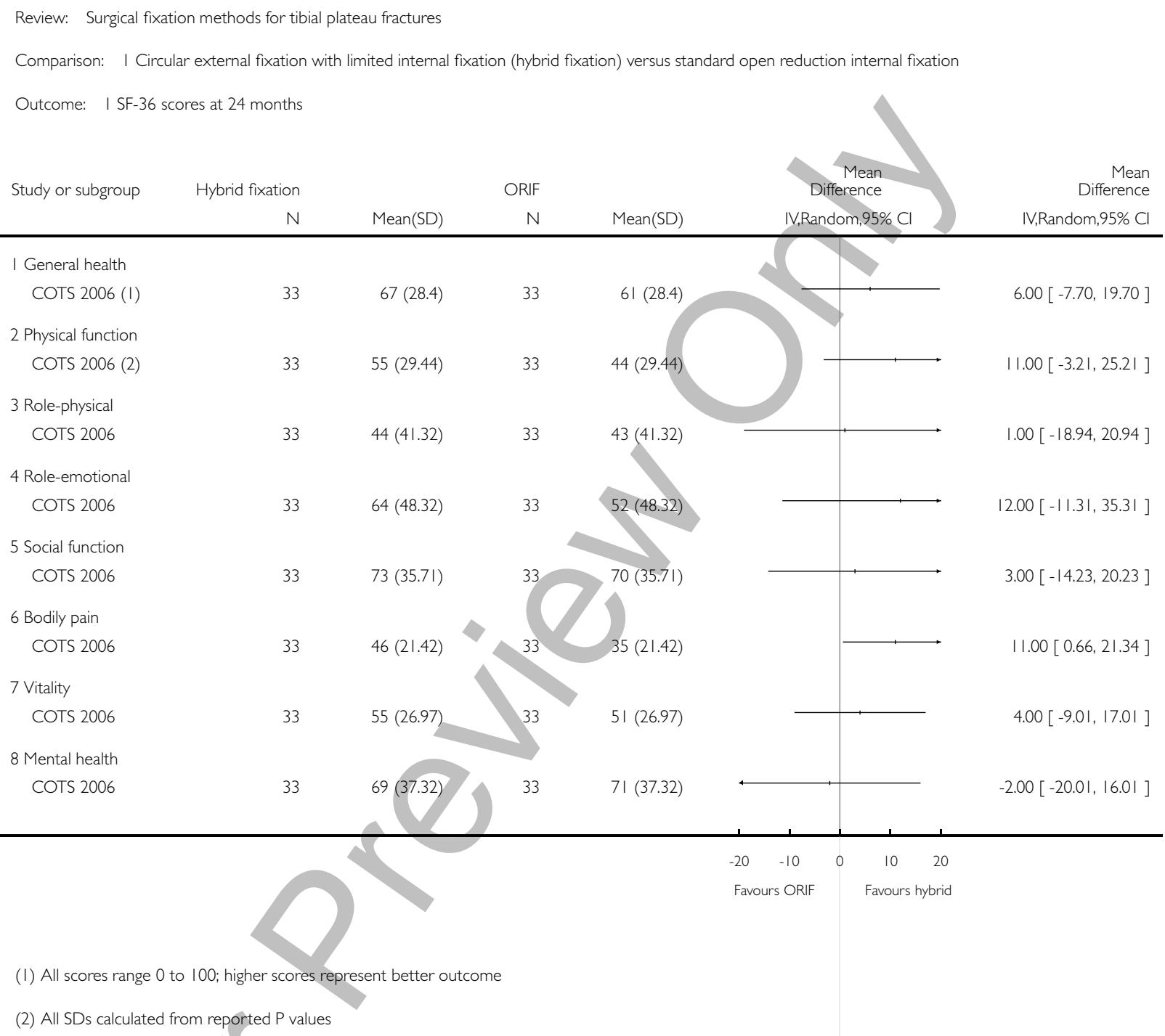

(2) All SDs calculated from reported $P$ values 
Analysis I.2. Comparison I Circular external fixation with limited internal fixation (hybrid fixation) versus standard open reduction internal fixation, Outcome 2 Hospital for Special Surgery Score.

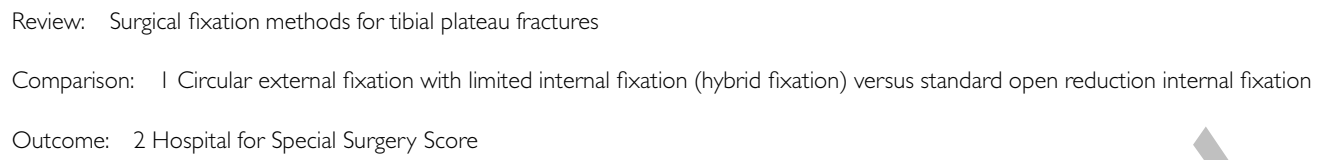

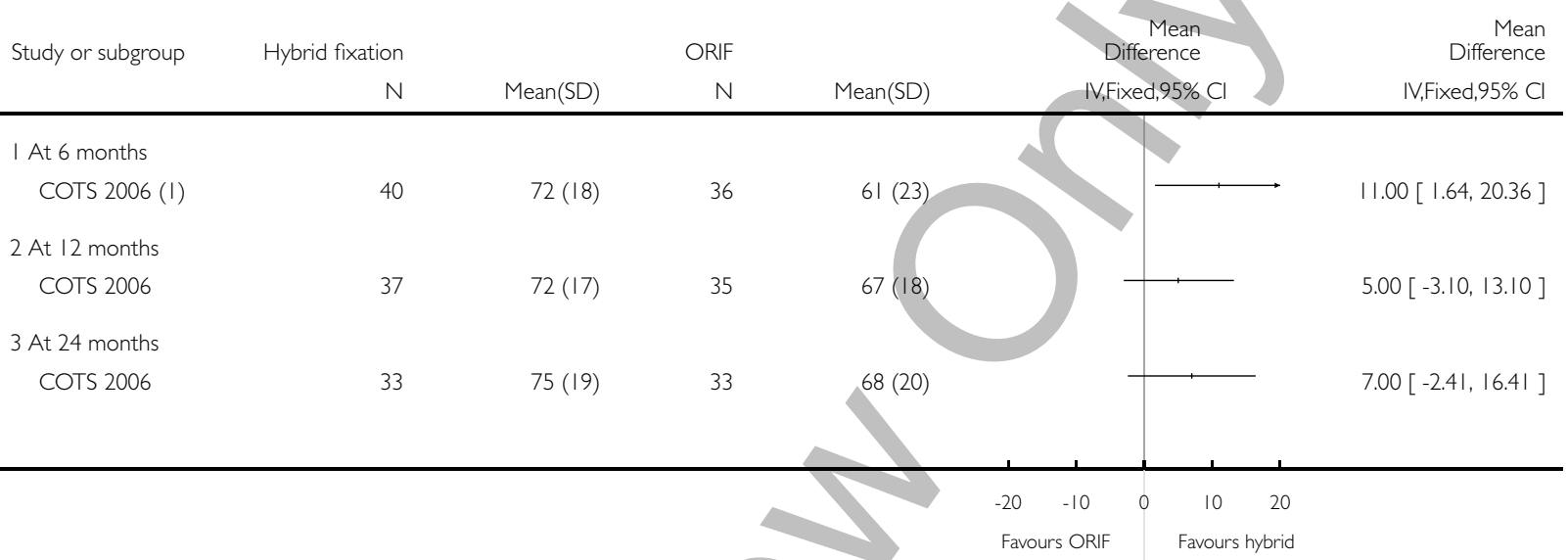

( I) HSS score (range 0 to 100). Higher scores represent better outcome. 
Analysis I.3. Comparison I Circular external fixation with limited internal fixation (hybrid fixation) versus standard open reduction internal fixation, Outcome 3 WOMAC Pain score.

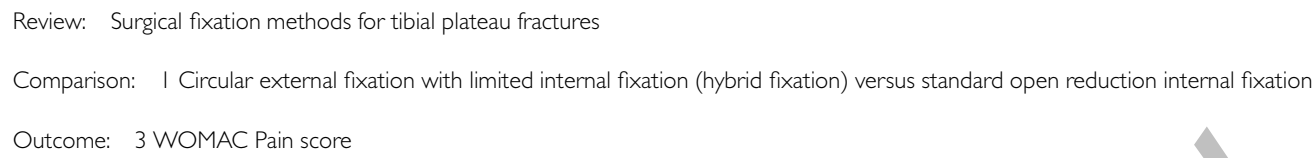


Analysis I.4. Comparison I Circular external fixation with limited internal fixation (hybrid fixation) versus standard open reduction internal fixation, Outcome 4 WOMAC Stiffness score.

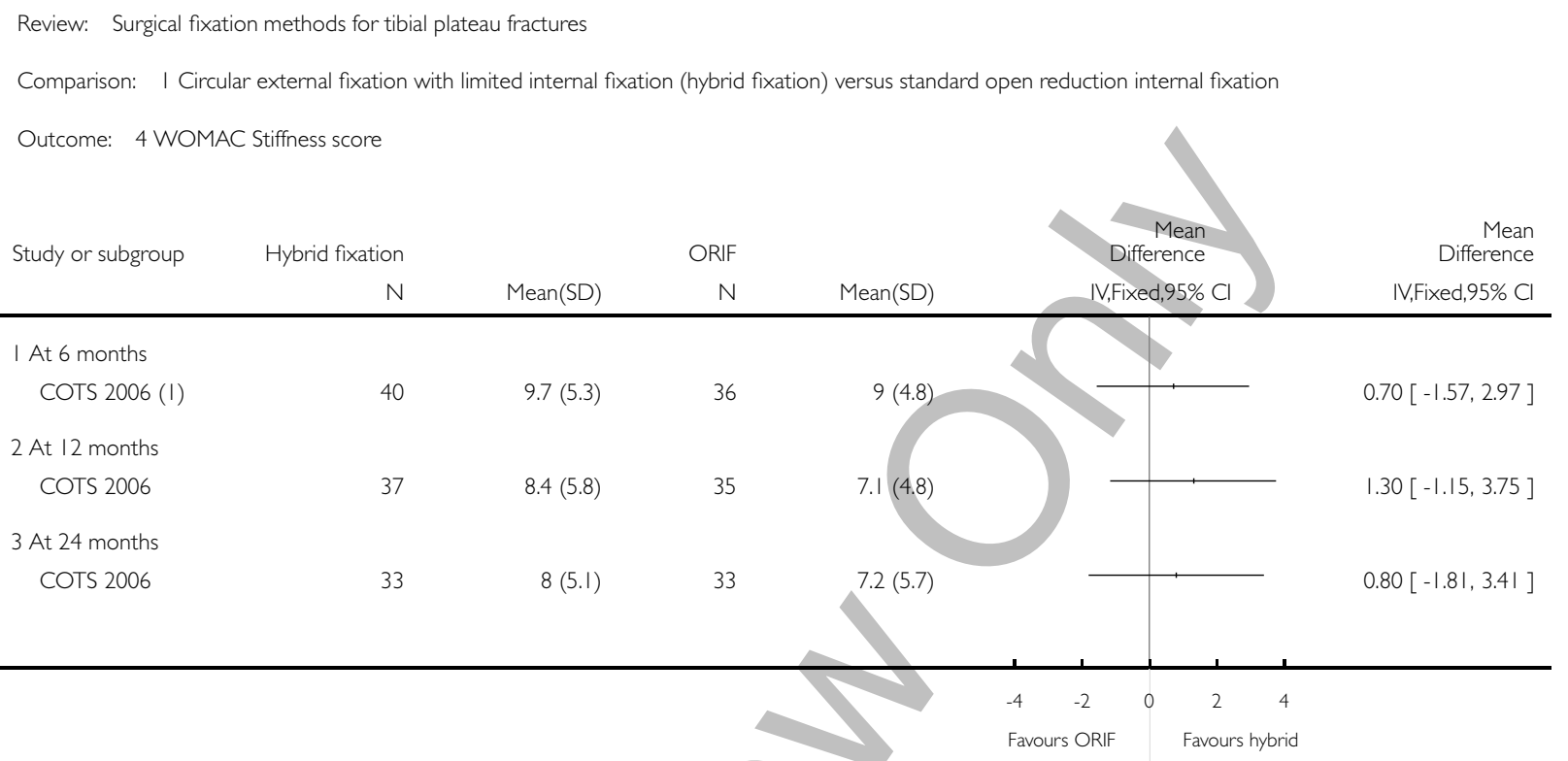

(1) Assumption score ranges 0 to 100; higher scores indicate greater stiffness 
Analysis I.5. Comparison I Circular external fixation with limited internal fixation (hybrid fixation) versus standard open reduction internal fixation, Outcome 5 WOMAC Function score.

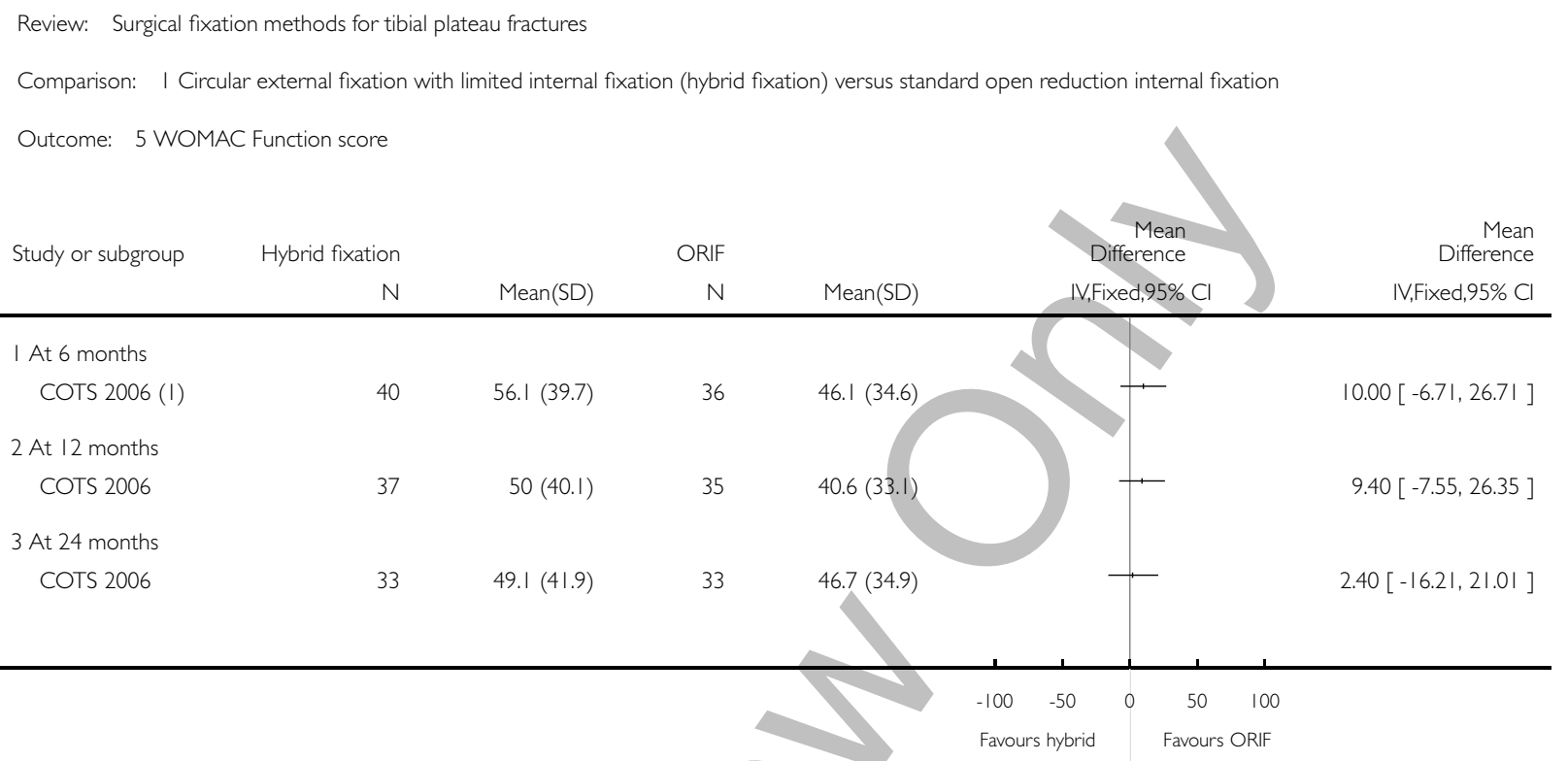

(I) Assumption score ranges 0 to 100; higher scores indicate greater functional limitation 
Analysis I.6. Comparison I Circular external fixation with limited internal fixation (hybrid fixation) versus standard open reduction internal fixation, Outcome 6 Reoperations (24 months).

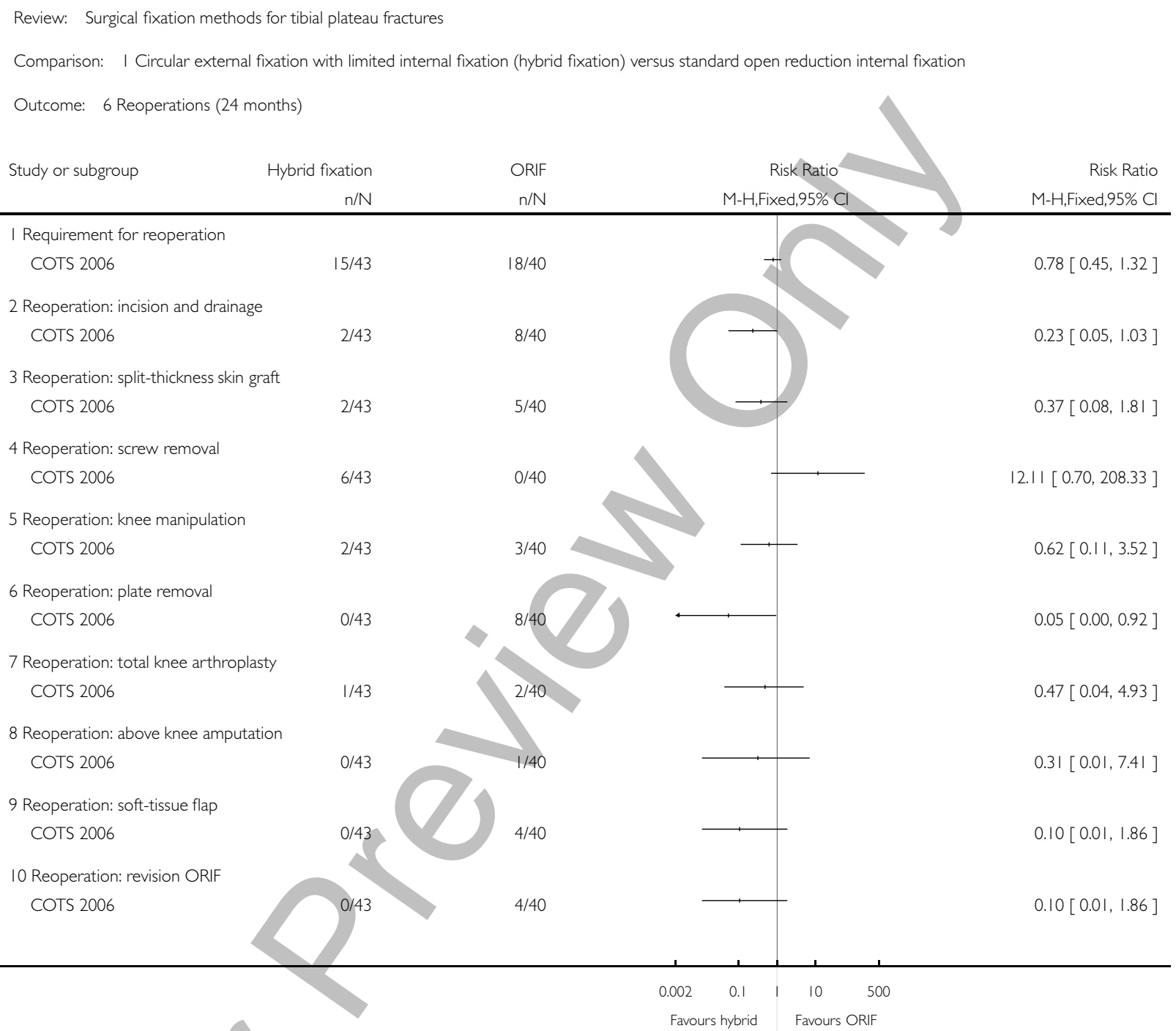


Analysis I.7. Comparison I Circular external fixation with limited internal fixation (hybrid fixation) versus standard open reduction internal fixation, Outcome 7 Intra-operative measures (length of surgery, blood loss).

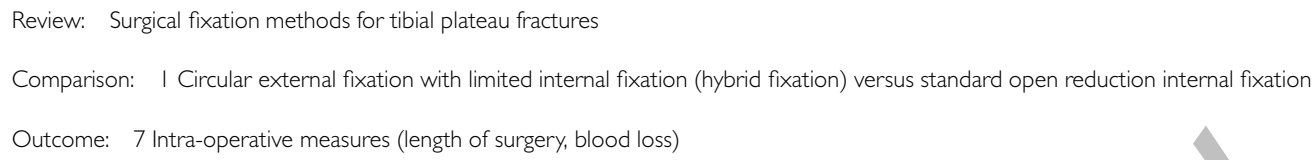

\begin{tabular}{llllll}
$\mathrm{N}$ & Mean(SD) & $\mathrm{N}$ & Mean(SD) & $\mathrm{IV}$, Fixed,95\% Cl & $\mathrm{IV}, \mathrm{Fixed}, 95 \% \mathrm{Cl}$ \\
\hline
\end{tabular}

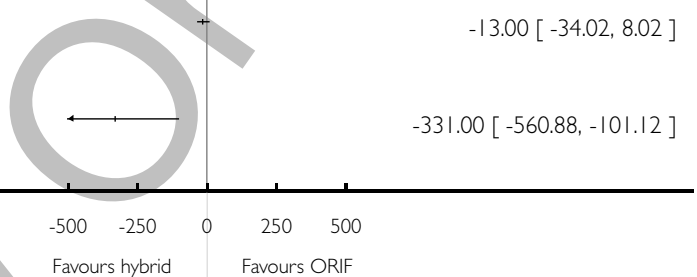

(I) SDs calculated from reported $\mathrm{P}$ value

(2) SDs calculated from reported $\mathrm{P}$ value

Analysis I.8. Comparison I Circular external fixation with limited internal fixation (hybrid fixation) versus standard open reduction internal fixation, Outcome 8 Knee joint total range of motion (degrees) at 24 months.
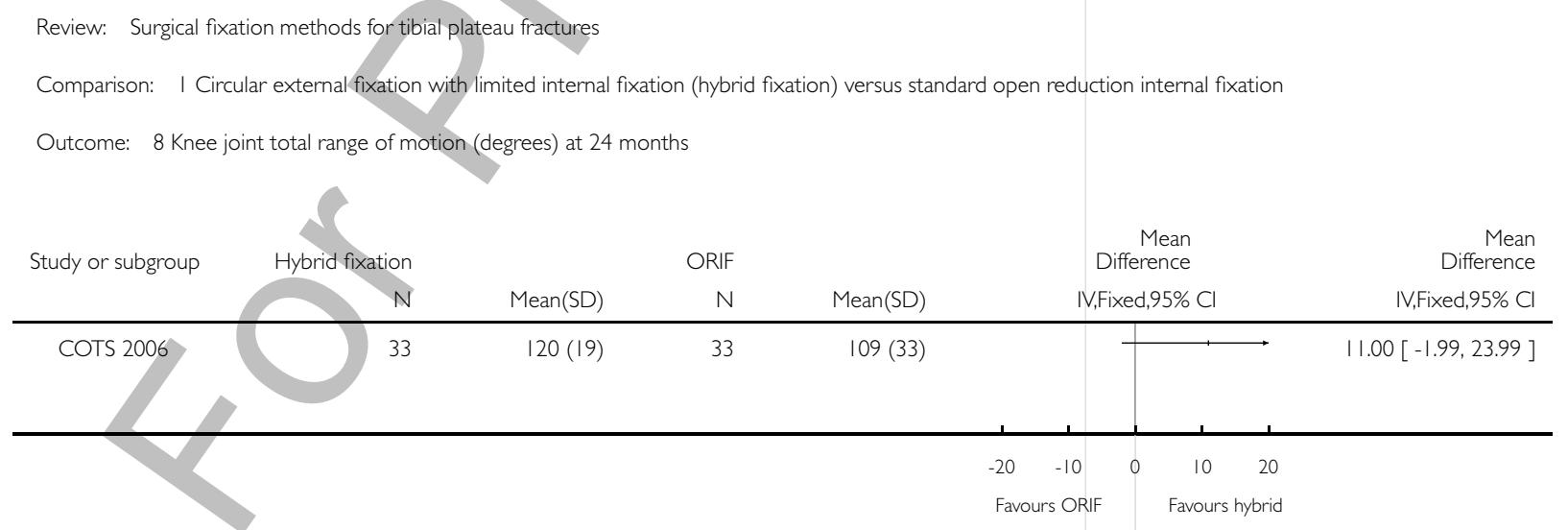
Analysis I.9. Comparison I Circular external fixation with limited internal fixation (hybrid fixation) versus standard open reduction internal fixation, Outcome 9 Return to pre-injury activity level.

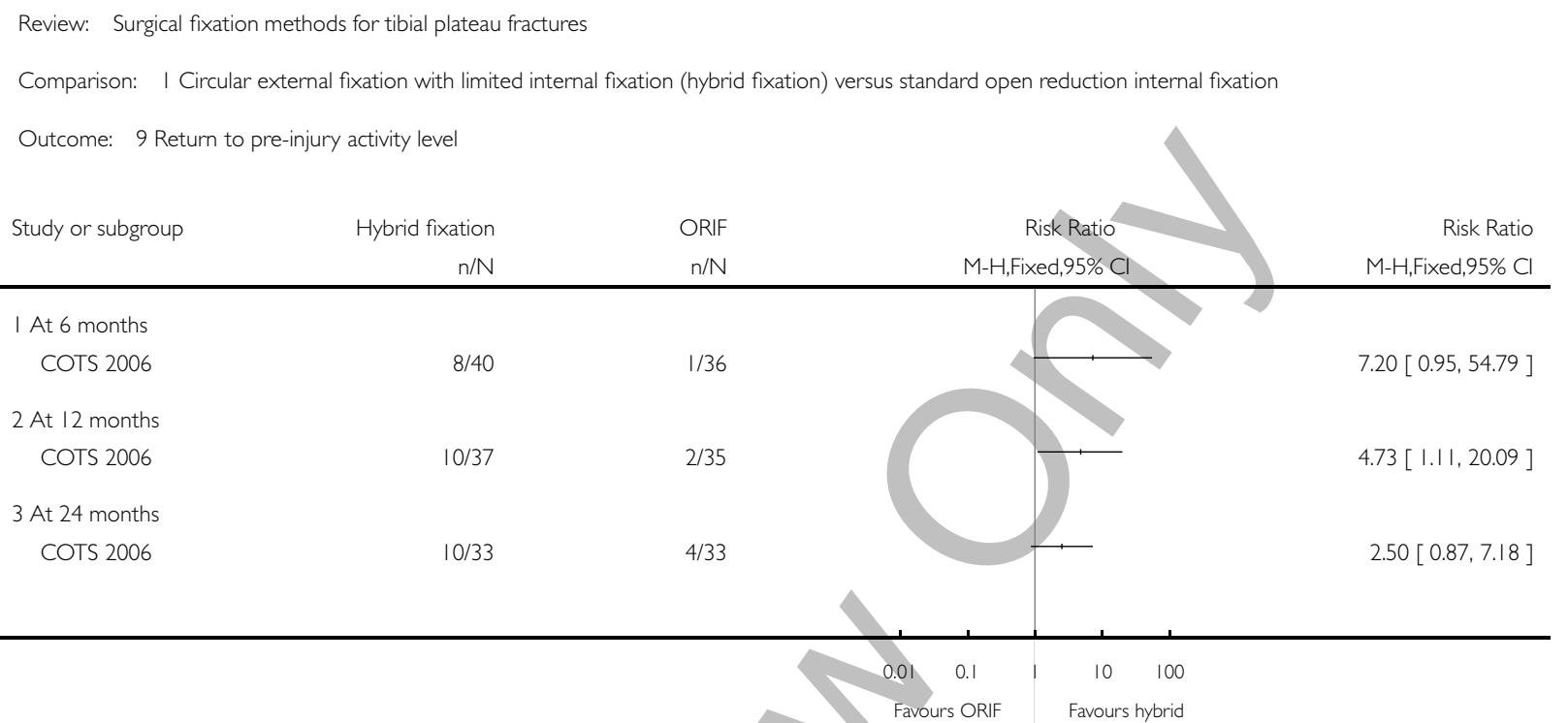

Analysis I.10. Comparison I Circular external fixation with limited internal fixation (hybrid fixation) versus standard open reduction internal fixation, Outcome 10 Length of hospital stay (days).
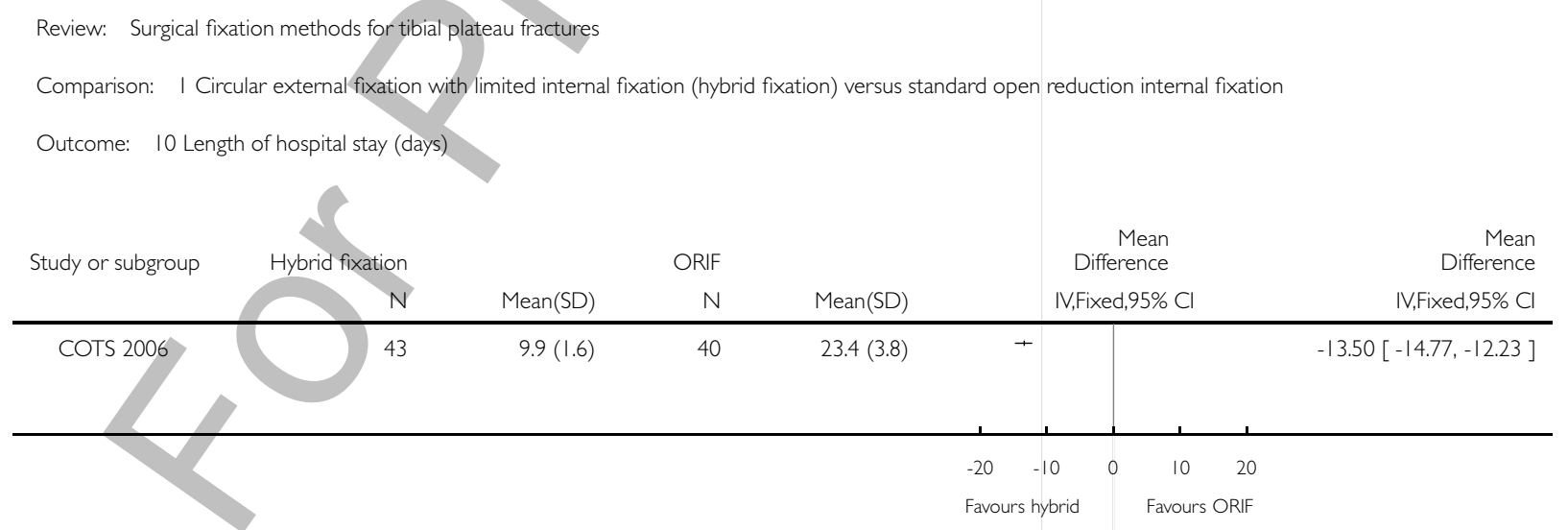
Analysis I.I I. Comparison I Circular external fixation with limited internal fixation (hybrid fixation) versus standard open reduction internal fixation, Outcome I I Radiological outcomes (postoperatively).

Review: Surgical fixation methods for tibial plateau fractures

Comparison: I Circular external fixation with limited internal fixation (hybrid fixation) versus standard open reduction internal fixation

Outcome: I Radiological outcomes (postoperatively)

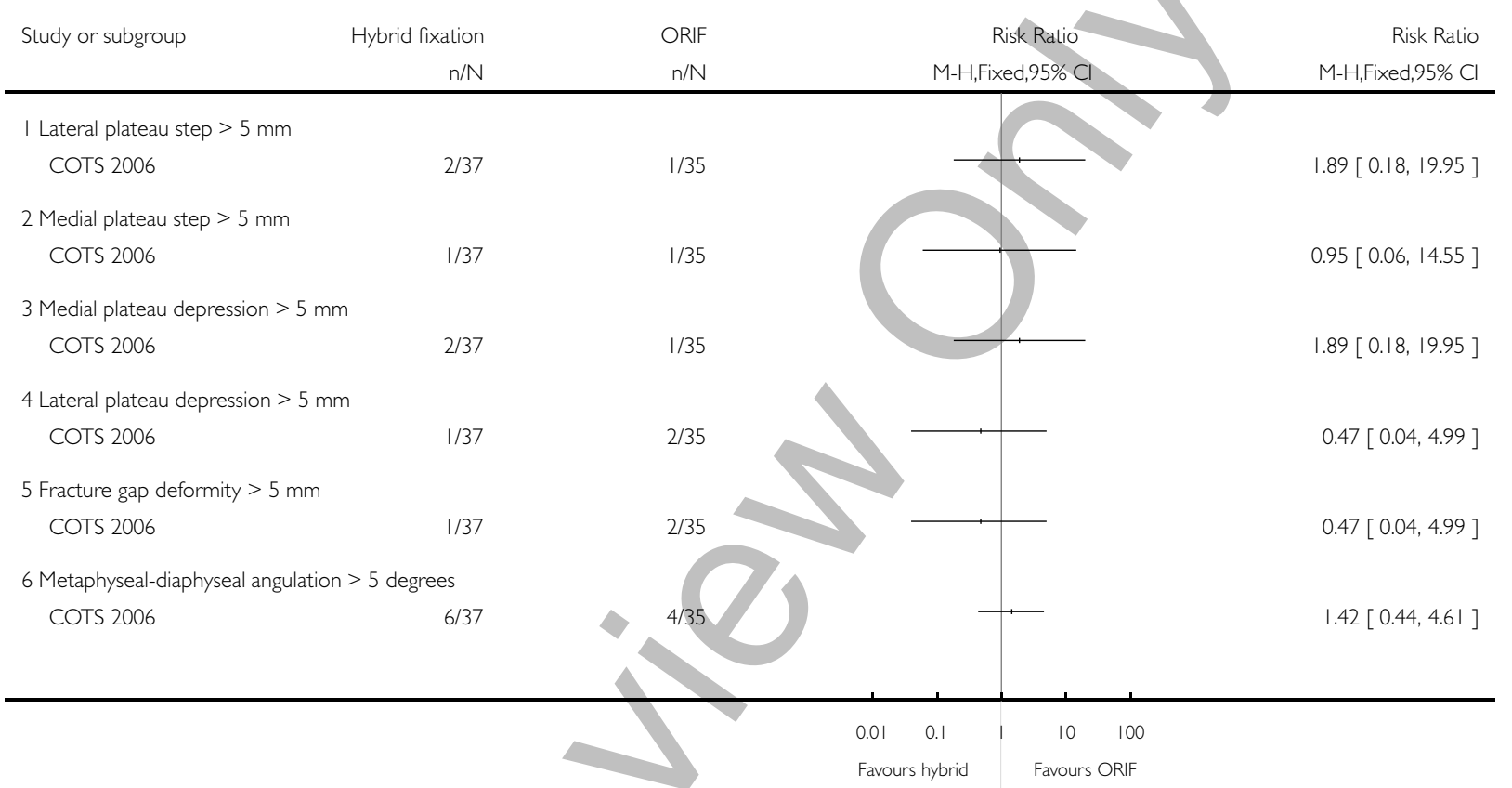


Analysis I.I2. Comparison I Circular external fixation with limited internal fixation (hybrid fixation) versus standard open reduction internal fixation, Outcome 12 Radiological evidence of tibiofemoral joint osteoarthritis.

Review: Surgical fixation methods for tibial plateau fractures

Comparison: I Circular external fixation with limited internal fixation (hybrid fixation) versus standard open reduction internal fixation

Outcome: 12 Radiological evidence of tibiofemoral joint osteoarthritis

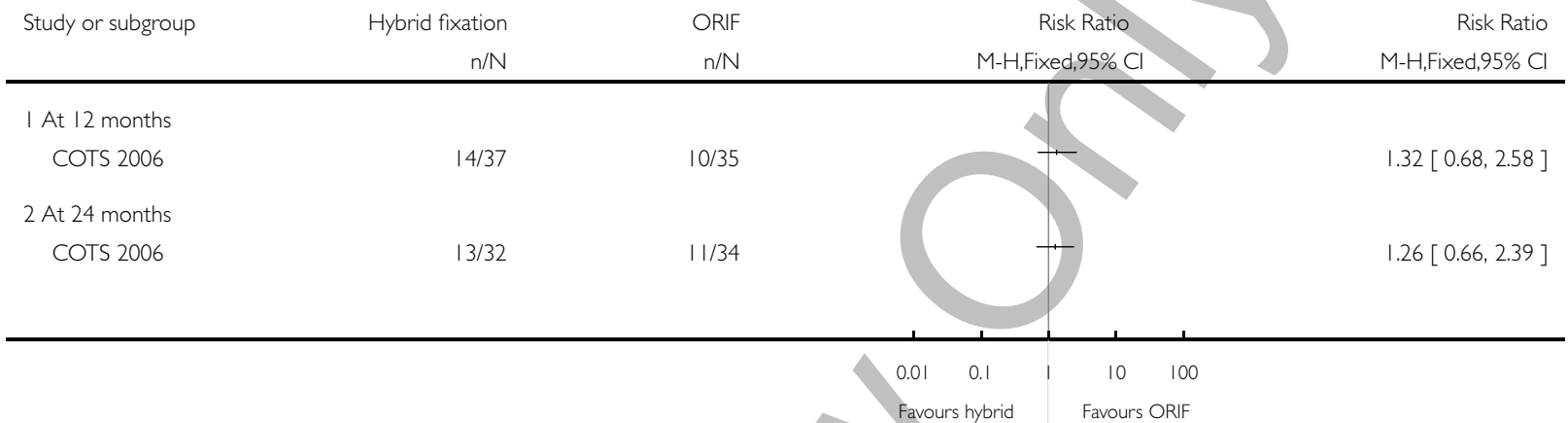

Analysis 2.I. Comparison 2 Less invasive stabilisation system (LISS) versus double-plating open reduction internal fixation, Outcome I Hospital for Special Surgery Score.

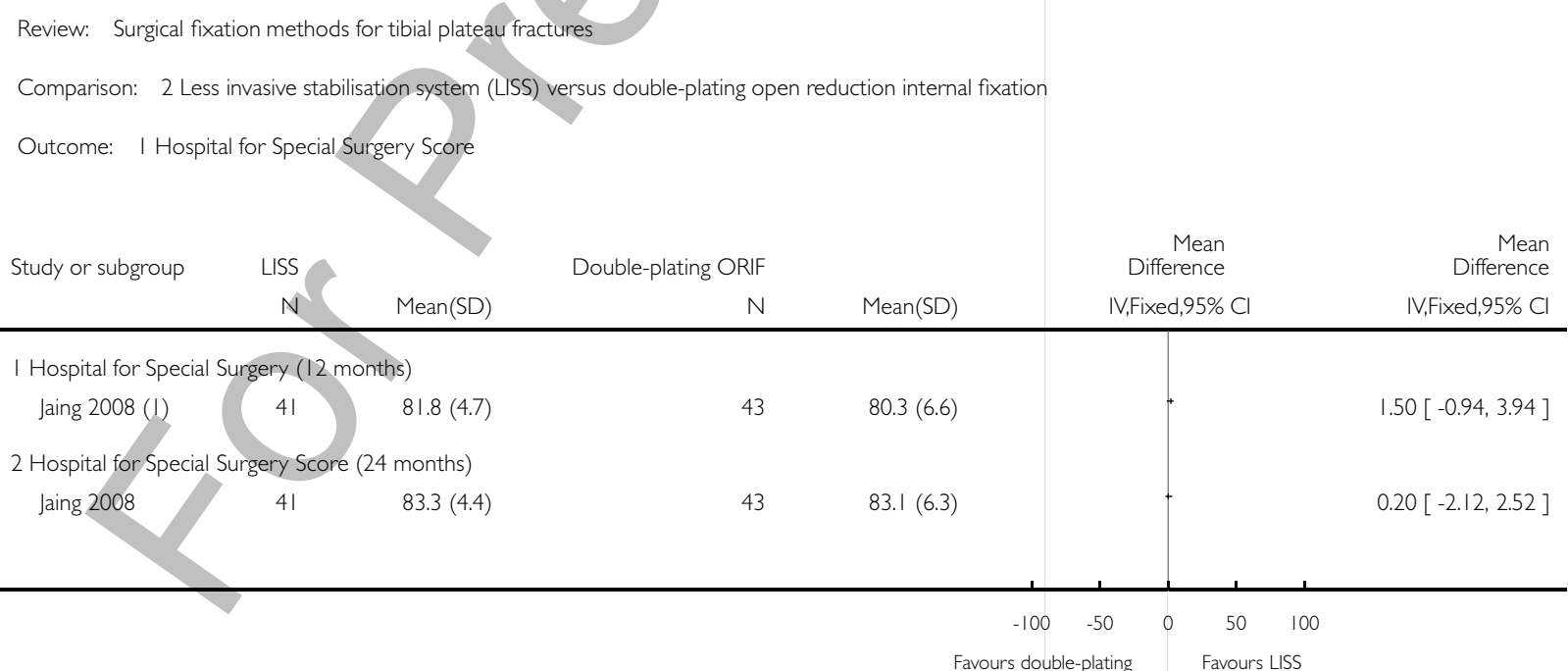

( I) HSS score (range 0 to 100). Higher scores represent better outcome. 
Analysis 2.2. Comparison 2 Less invasive stabilisation system (LISS) versus double-plating open reduction internal fixation, Outcome 2 Complications (24 months).

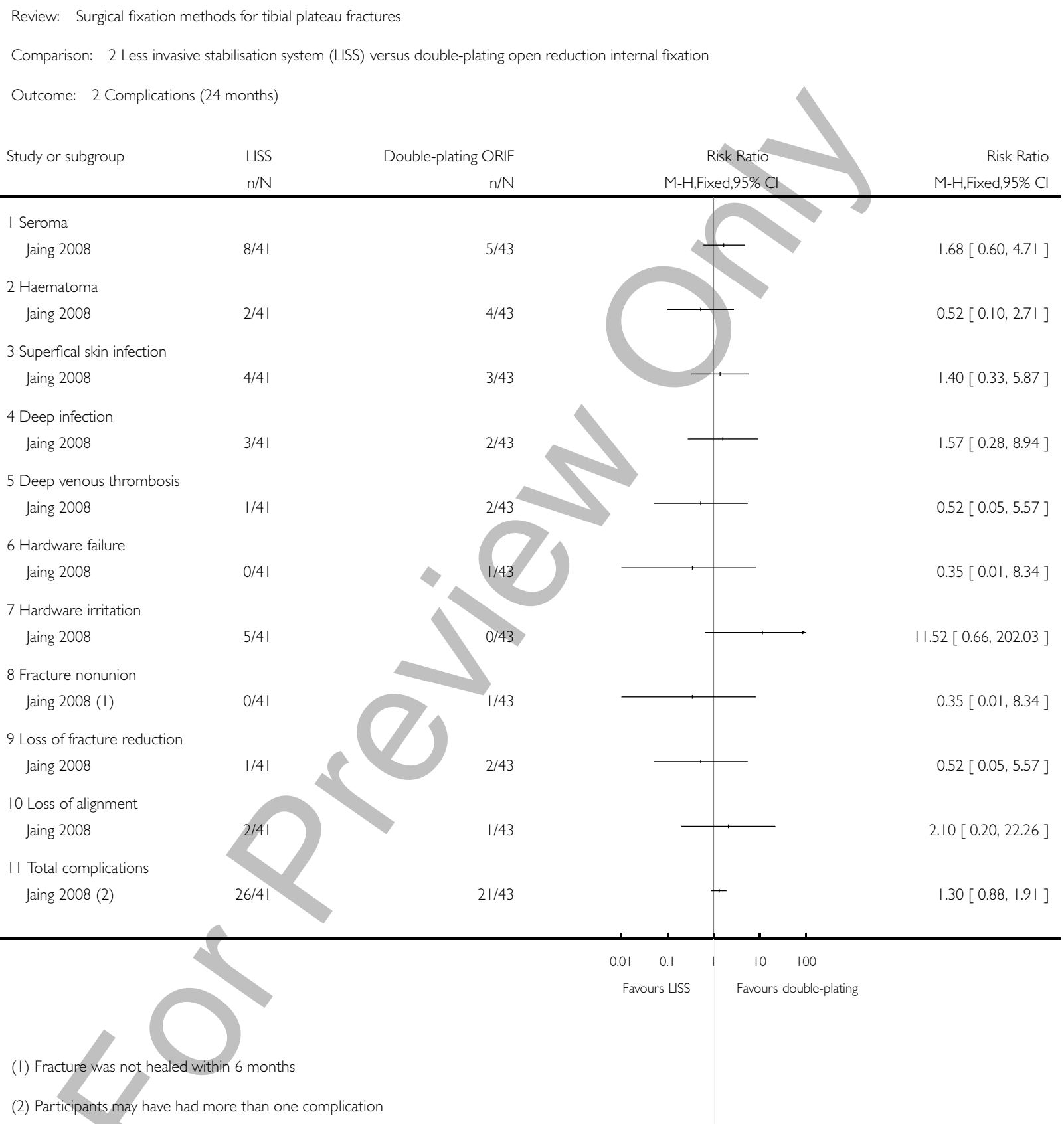


Analysis 2.3. Comparison 2 Less invasive stabilisation system (LISS) versus double-plating open reduction internal fixation, Outcome 3 Reoperations (up to 24 months).

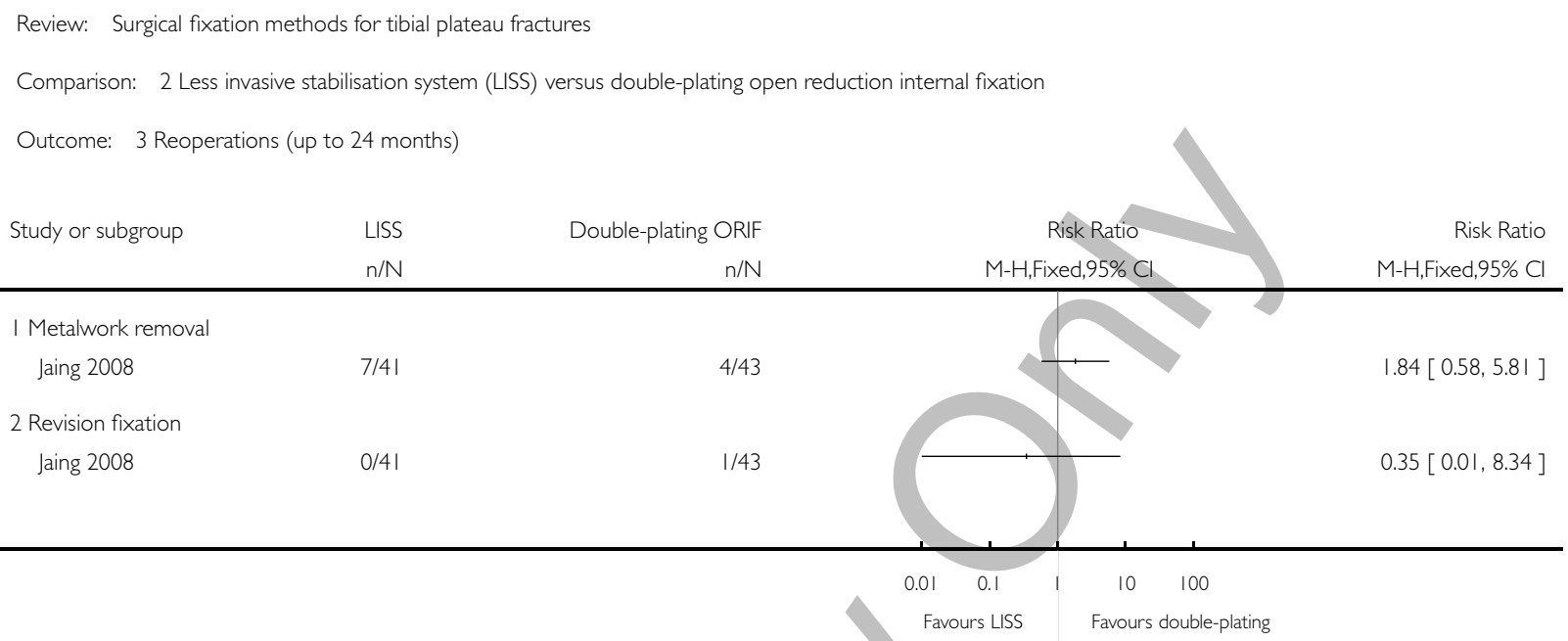

Analysis 2.4. Comparison 2 Less invasive stabilisation system (LISS) versus double-plating open reduction internal fixation, Outcome 4 Length of surgery $(\mathrm{min})$.

Review: Surgical fixation methods for tibial plateau fractures

Comparison: 2 Less invasive stabilisation system (LISS) versus double-plating open reduction internal fixation

Outcome: 4 Length of surgery (min)

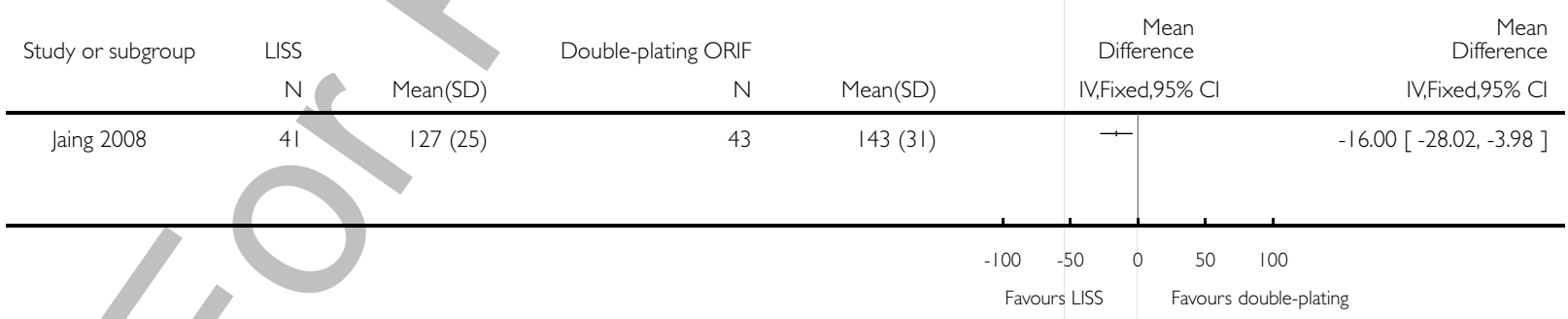


Analysis 2.5. Comparison 2 Less invasive stabilisation system (LISS) versus double-plating open reduction internal fixation, Outcome 5 Incision length $(\mathrm{cm})$.

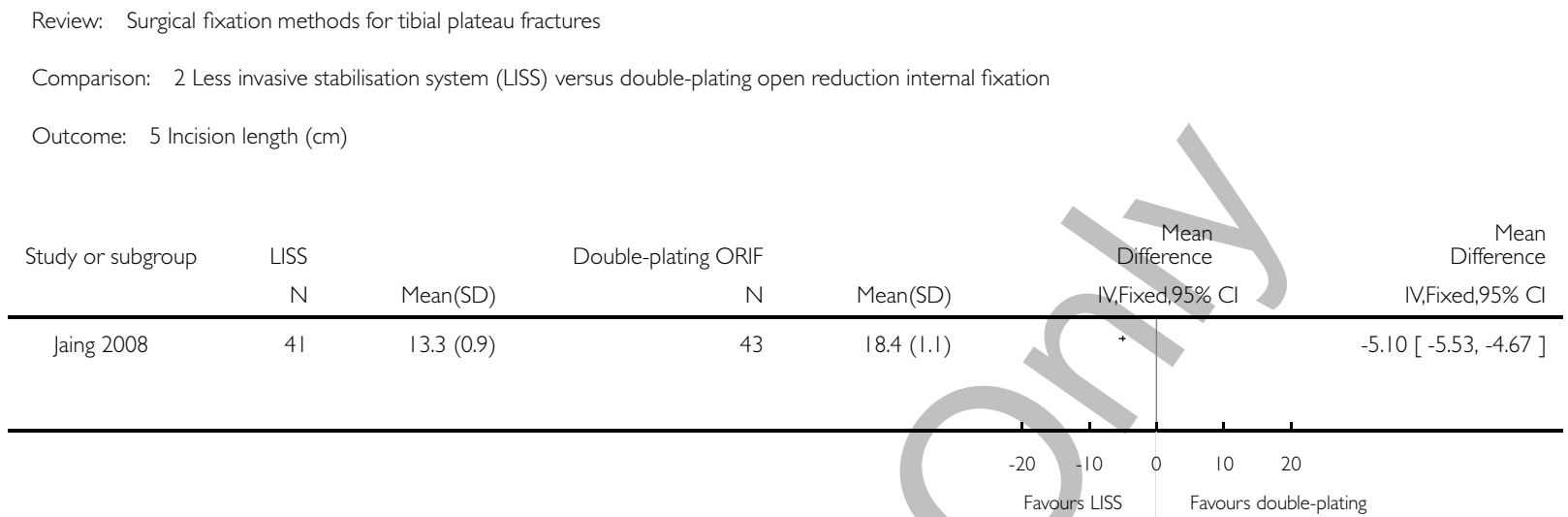

Analysis 2.6. Comparison 2 Less invasive stabilisation system (LISS) versus double-plating open reduction internal fixation, Outcome 6 Perioperative blood loss $(\mathrm{ml})$.

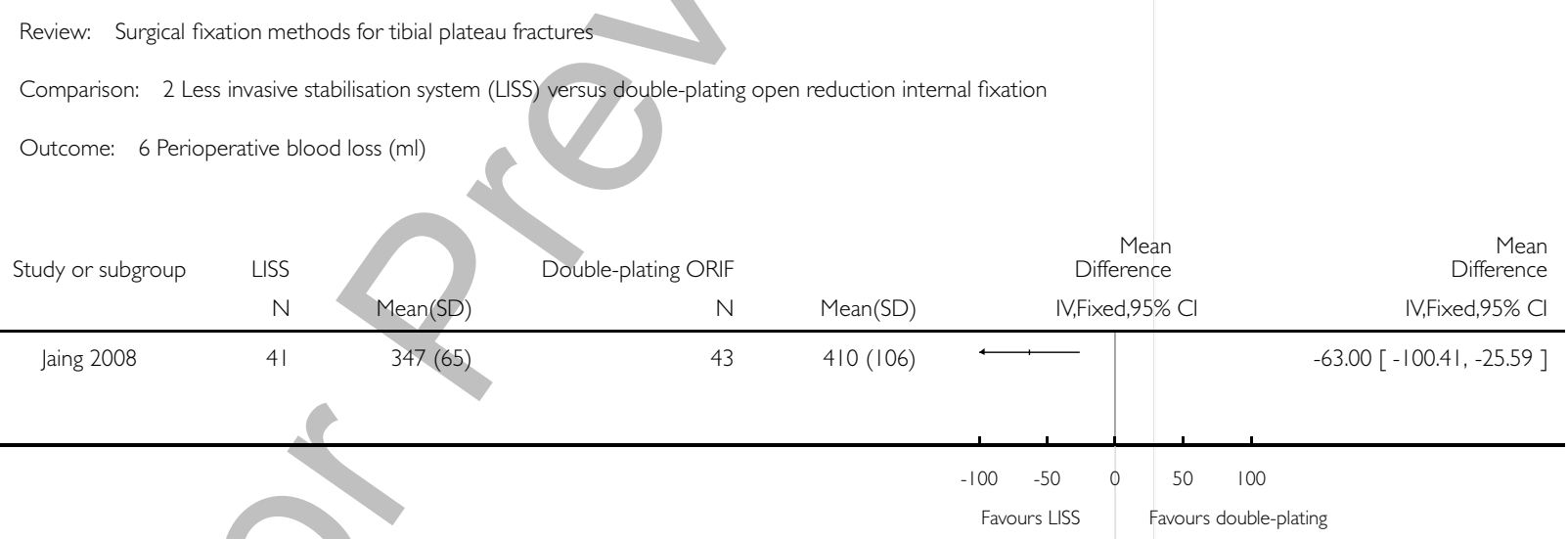


Analysis 2.7. Comparison 2 Less invasive stabilisation system (LISS) versus double-plating open reduction internal fixation, Outcome 7 Postsurgical malreduction and malalignment.

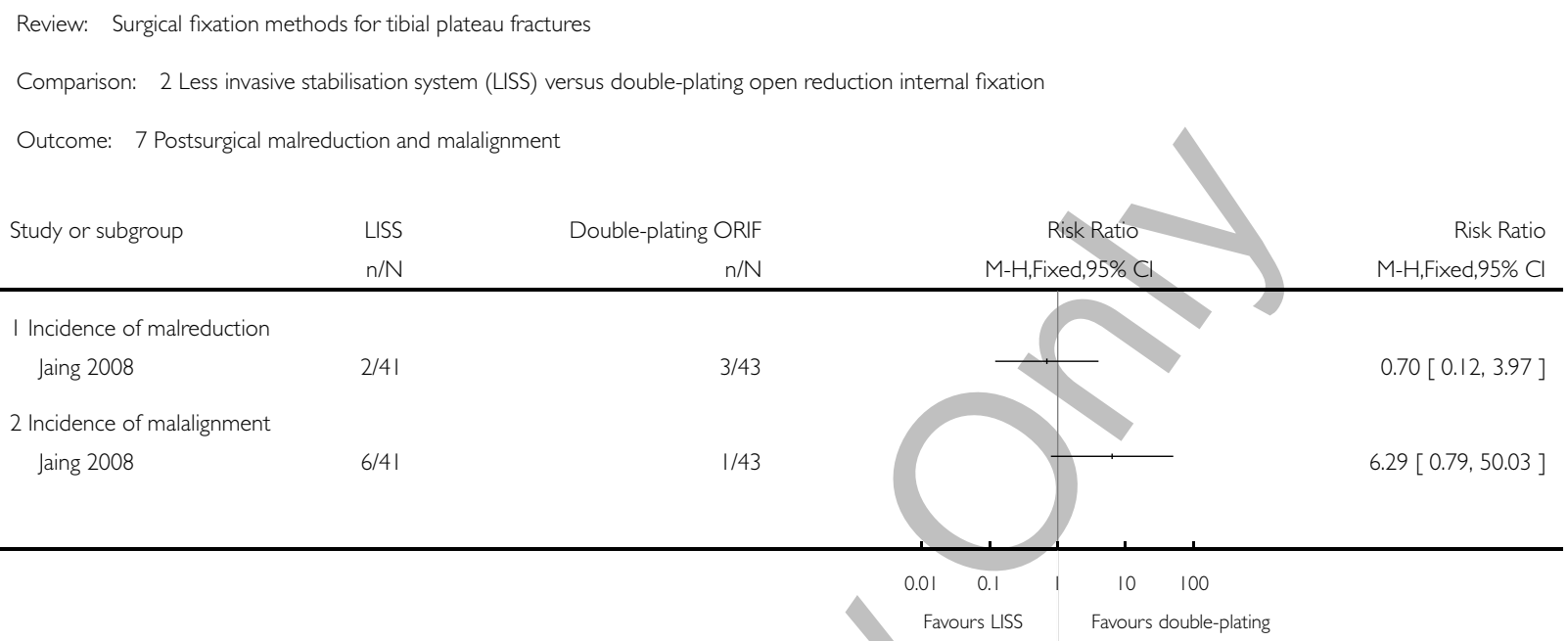

Analysis 2.8. Comparison 2 Less invasive stabilisation system (LISS) versus double-plating open reduction internal fixation, Outcome 8 Time to radiological union (weeks).

Review: Surgical fixation methods for tibial plateau fractures

Comparison: 2 Less invasive stabilisation system (LISS) versus double-plating open reduction internal fixation

Outcome: 8 Time to radiological union (weeks)

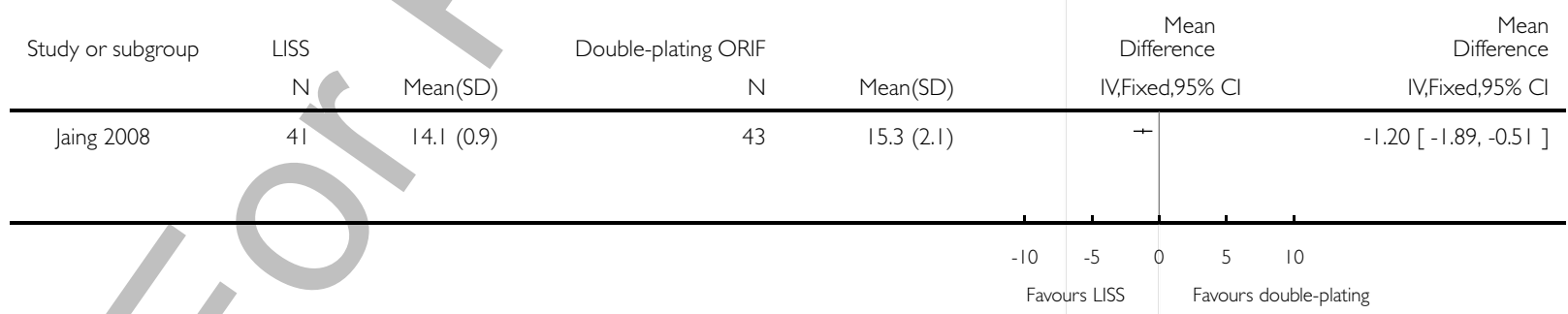


Analysis 3.I. Comparison 3 Arthroscopically assisted percutaneous reduction and internal fixation versus standard open reduction and internal fixation, Outcome I Hospital for Special Surgery Score (I4 months).

Review: Surgical fixation methods for tibial plateau fractures

Comparison: 3 Arthroscopically assisted percutaneous reduction and internal fixation versus standard open reduction and internal fixation

Outcome: I Hospital for Special Surgery Score (14 months)

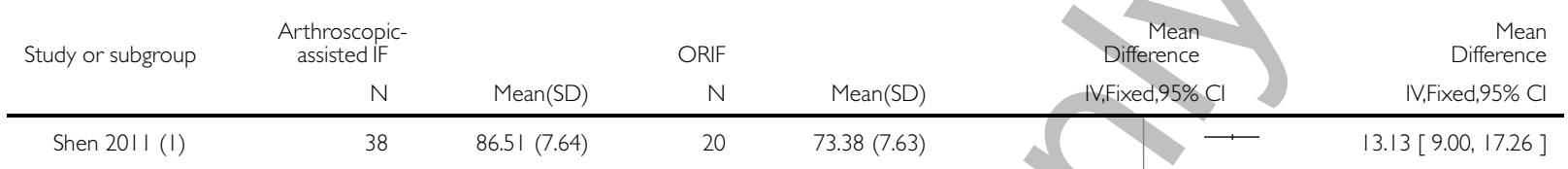

( I) HSS score (range 0 to 100). Higher scores represent better outcome.

Analysis 3.2. Comparison 3 Arthroscopically assisted percutaneous reduction and internal fixation versus standard open reduction and internal fixation, Outcome 2 Knee stiffness.

Review: Surgical fixation methods for tibial plateau fractures

Comparison: 3 Arthroscopically assisted percutaneous reduction and internal fixation versus standard open reduction and internal fixation

Outcome: 2 Knee stiffness

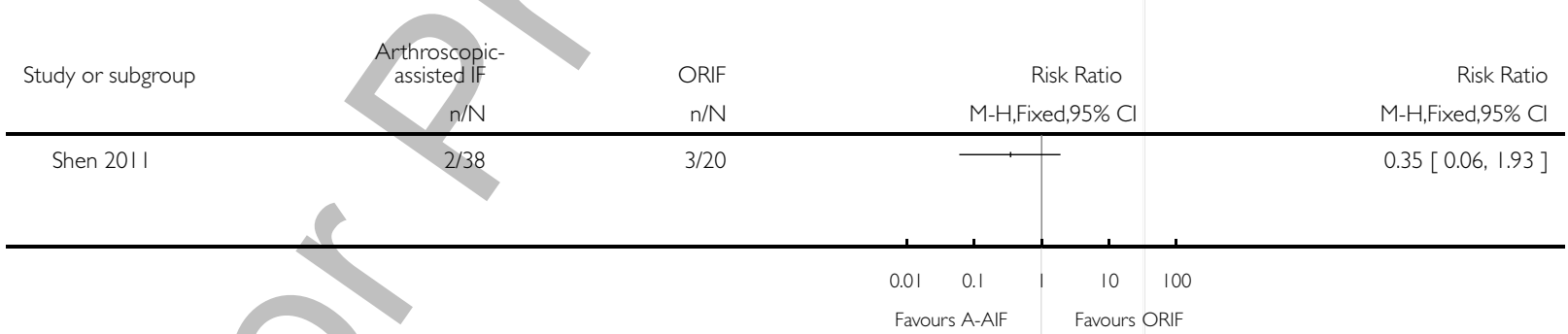


Analysis 3.3. Comparison 3 Arthroscopically assisted percutaneous reduction and internal fixation versus standard open reduction and internal fixation, Outcome 3 Length of surgery ( $\mathrm{min})$.

Review: Surgical fixation methods for tibial plateau fractures

Comparison: 3 Arthroscopically assisted percutaneous reduction and internal fixation versus standard open reduction and internal fixation

Outcome: 3 Length of surgery $(\mathrm{min})$

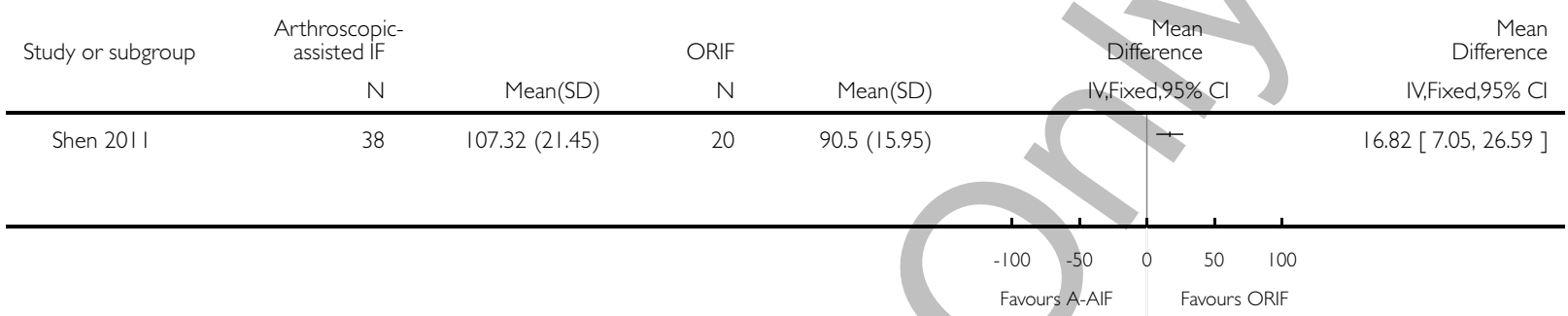

Analysis 3.4. Comparison 3 Arthroscopically assisted percutaneous reduction and internal fixation versus standard open reduction and internal fixation, Outcome 4 Incision length $(\mathrm{cm})$.

Review: Surgical fixation methods for tibial plateau fractures

Comparison: 3 Arthroscopically assisted percutaneous reduction and internal fixation versus standard open reduction and internal fixation

Outcome: 4 Incision length $(\mathrm{cm})$

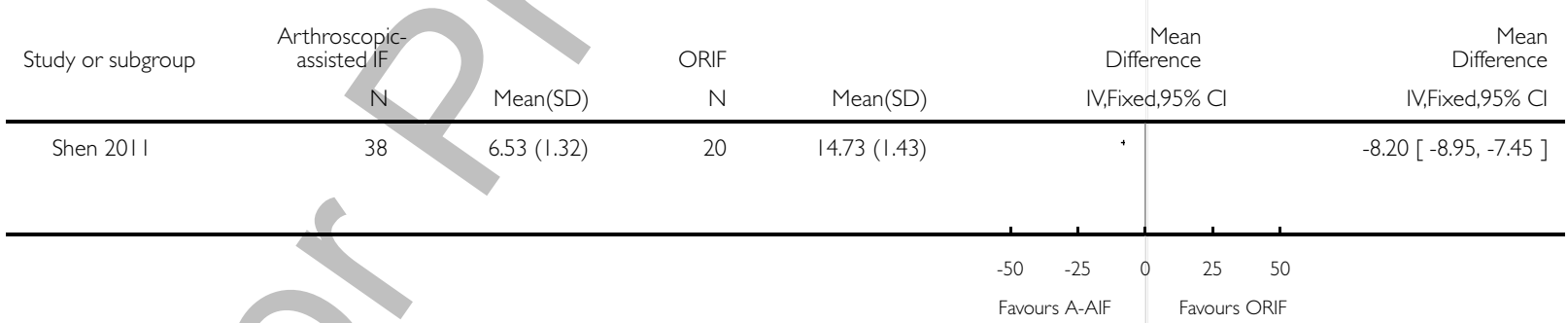


Analysis 3.5. Comparison 3 Arthroscopically assisted percutaneous reduction and internal fixation versus standard open reduction and internal fixation, Outcome 5 Knee joint range of motion (degrees) (I4 months).

Review: Surgical fixation methods for tibial plateau fractures

Comparison: 3 Arthroscopically assisted percutaneous reduction and internal fixation versus standard open reduction and internal fixation

Outcome: 5 Knee joint range of motion (degrees) (14 months)

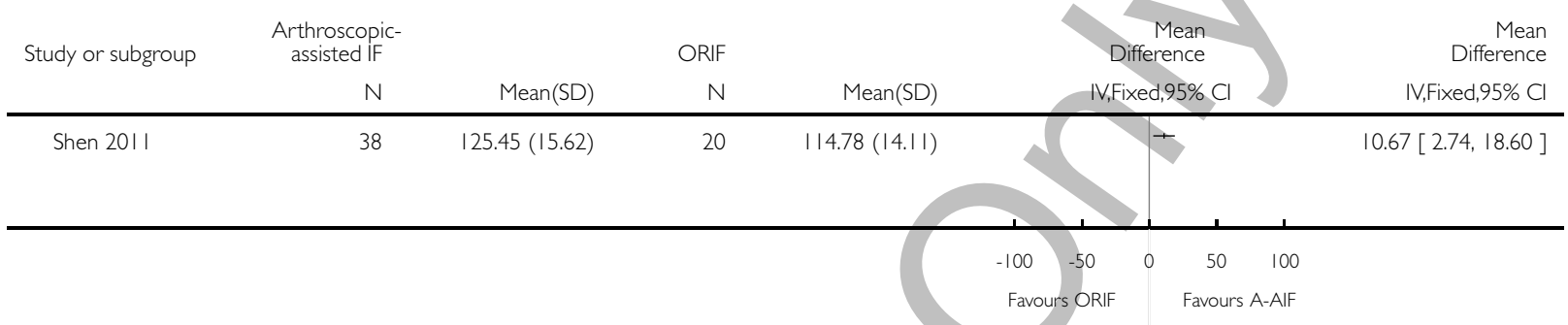

Analysis 3.6. Comparison 3 Arthroscopically assisted percutaneous reduction and internal fixation versus standard open reduction and internal fixation, Outcome 6 Time to radiological union (months).

Review: Surgical fixation methods for tibial plateau fractures

Comparison: 3 Arthroscopically assisted percutaneous reduction and internal fixation versus standard open reduction and internal fixation

Outcome: 6 Time to radiological union (months)

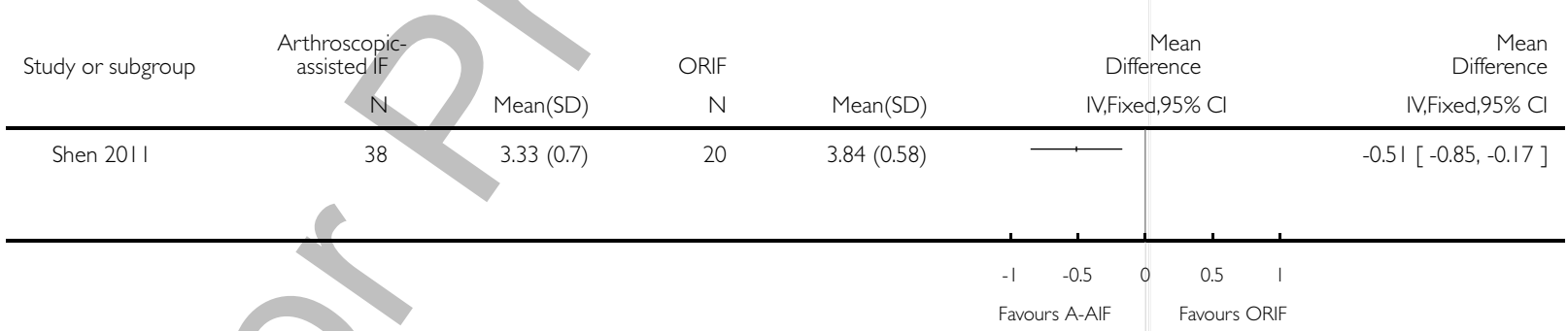


Analysis 4.I. Comparison 4 Bone substitute versus autologous bone graft, Outcome I Lower limb function.

Review: Surgical fixation methods for tibial plateau fractures

Comparison: 4 Bone substitute versus autologous bone graft

Outcome: I Lower limb function

Study or subgroup

Bone substiutute

Autogenous graft

Risk Ratio

Risk Ratio

$\mathrm{n} / \mathrm{N}$

$\mathrm{n} / \mathrm{N}$

M-H,Fixed, $95 \%$ Cl

M-H,Fixed,95\% Cl

| Ability to walk (excellent or good)

Heikkilä 201 I

$13 / 14$

$10 / 11$

2 Stair climbing (excellent or good)

Heikkilä 20। I

$11 / 14$

$10 / 11$

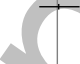

3 Squatting (excellent or good)

Heikkilä 20। I

$12 / 14$

$9 / 11$

4 Jumping (excellent or good)

Heikkilä 20। I

$6 / 14$

$4 / 11$

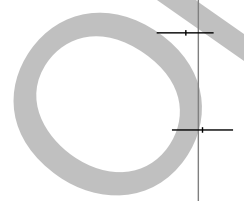

$0.86[0.62,1.20]$

$1.05[0.74,1.49]$

$1.18[0.44,3.17]$ 
Analysis 4.2. Comparison 4 Bone substitute versus autologous bone graft, Outcome 2 Adverse events.

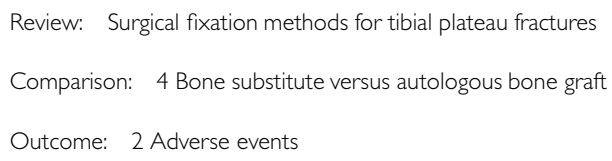

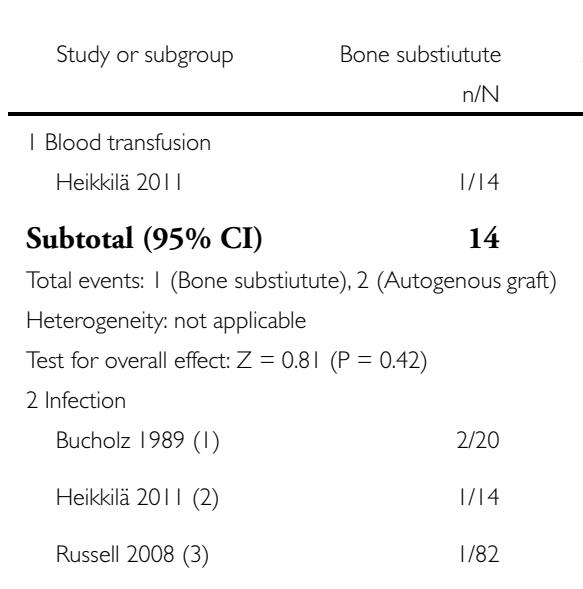

$\mathrm{n} / \mathrm{N}$
M-H,Fixed,95\% C M-H,Fixed,95\% Cl

\section{Subtotal $(95 \%$ CI)}

Total events: 4 (Bone substiutute), 3 (Autogenous graft) Heterogeneity: $\mathrm{Ch}^{2}=0.6 \mathrm{l}, \mathrm{df}=2(\mathrm{P}=0.74) ; \mathrm{I}^{2}=0.0 \%$ Test for overall effect: $Z=0.02(P=0.99)$ 3 Nonunion

Russell 2008

Subtotal $(\mathbf{9 5 \%}$ CI)

Total events: 0 (Bone substiutute), 0 (Autogenous graft) Heterogeneity: not applicable

Test for overall effect: not applicable

4 Implant breakage or deformity

$$
\text { Russell } 2008
$$

$2 / 11$

11

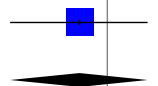

$2 / 20$

$0 / 11$

$1 / 38$

69

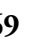

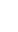
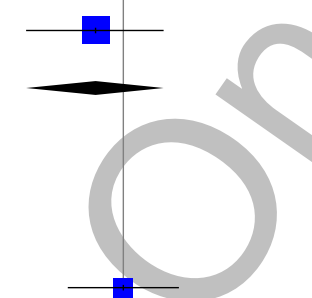

$100.0 \%$

$0.39[0.04,3.79]$

$100.0 \%$

0.39 [ 0.04, 3.79]

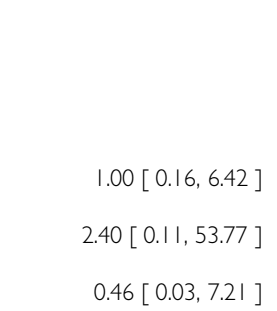

$1.01[0.27,3.82]$

Not estimable

Not estimable

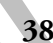

$51.0 \%$

$14.2 \%$

$100.0 \%$

\author{
$1.01[0.27,3.82]$
}

Subtotal (95\% CI)

82

$0 / 38$

38

Total events: 0 (Bone substiutute), 0 (Autogenous graft)

Heterogeneity: not applicable

Test for overall effect: not applicable

5 Prominent metalwork

Bucholz 1989

Subtotal $(95 \% \mathrm{CI})$

Total events: 3 (Bone substiutute), 3 (Autogenous graft)

Heterogeneity: not applicable

Test for overall effect: $Z=0.0(P=1.0)$

6 Loose screws 


\begin{tabular}{|c|c|c|c|c|c|}
\hline Study or subgroup & $\begin{array}{r}\text { Bone substiutute } \\
\mathrm{n} / \mathrm{N}\end{array}$ & $\begin{array}{r}\text { Autogenous graft } \\
n / N\end{array}$ & $\begin{array}{c}\text { Risk Ratio } \\
\text { M-H,Fixed,95\% Cl }\end{array}$ & Weight & $\begin{array}{r}\text { (. . . Continued }) \\
\text { Risk Ratio } \\
\text { M-H,Fixed,95\% Cl }\end{array}$ \\
\hline Bucholz 1989 & $1 / 20$ & $3 / 20$ & & $100.0 \%$ & $0.33[0.04,2.94]$ \\
\hline Subtotal $(95 \% \mathrm{CI})$ & 20 & 20 & & $100.0 \%$ & $0.33[0.04,2.94]$ \\
\hline \multicolumn{6}{|c|}{ Total events: I (Bone substiutute), 3 (Autogenous graft) } \\
\hline \multicolumn{6}{|c|}{ Heterogeneity: not applicable } \\
\hline \multicolumn{6}{|c|}{ Test for overall effect: $Z=0.99(P=0.32)$} \\
\hline \multicolumn{6}{|c|}{7 Donor site complication (infection) } \\
\hline Bucholz 1989 & 0/20 & $0 / 20$ & & & Not estimable \\
\hline Russell 2008 & $0 / 82$ & 0/38 & & & Not estimable \\
\hline Subtotal $(95 \% \mathrm{CI})$ & 102 & 58 & & & Not estimable \\
\hline \multicolumn{6}{|c|}{ Total events: 0 (Bone substiutute), 0 (Autogenous graft) } \\
\hline \multicolumn{6}{|c|}{ Heterogeneity: not applicable } \\
\hline \multicolumn{6}{|c|}{ Test for overall effect: not applicable } \\
\hline \multicolumn{6}{|c|}{8 Reoperation (for complication) } \\
\hline Bucholz 1989 (4) & $2 / 20$ & 2/20 & & $100.0 \%$ & $1.00[0.16,6.42]$ \\
\hline Subtotal $(95 \% \mathrm{CI})$ & 20 & 20 & & $100.0 \%$ & $1.00[0.16,6.42]$ \\
\hline \multicolumn{6}{|c|}{ Total events: 2 (Bone substiutute), 2 (Autogenous graft) } \\
\hline \multicolumn{6}{|c|}{ Heterogeneity: not applicable } \\
\hline \multicolumn{6}{|c|}{ Test for overall effect: $Z=0.0(P=1.0)$} \\
\hline
\end{tabular}

(I) All serious / deep infections resulted in eventual re-operation

(2) Superficial infection treated with debridement

(3) Surgical site infections resolved with wound care and antibiotics

(4) All re-operations were related to infection 
Analysis 4.3. Comparison 4 Bone substitute versus autologous bone graft, Outcome 3 Range of movement (at 12 months).

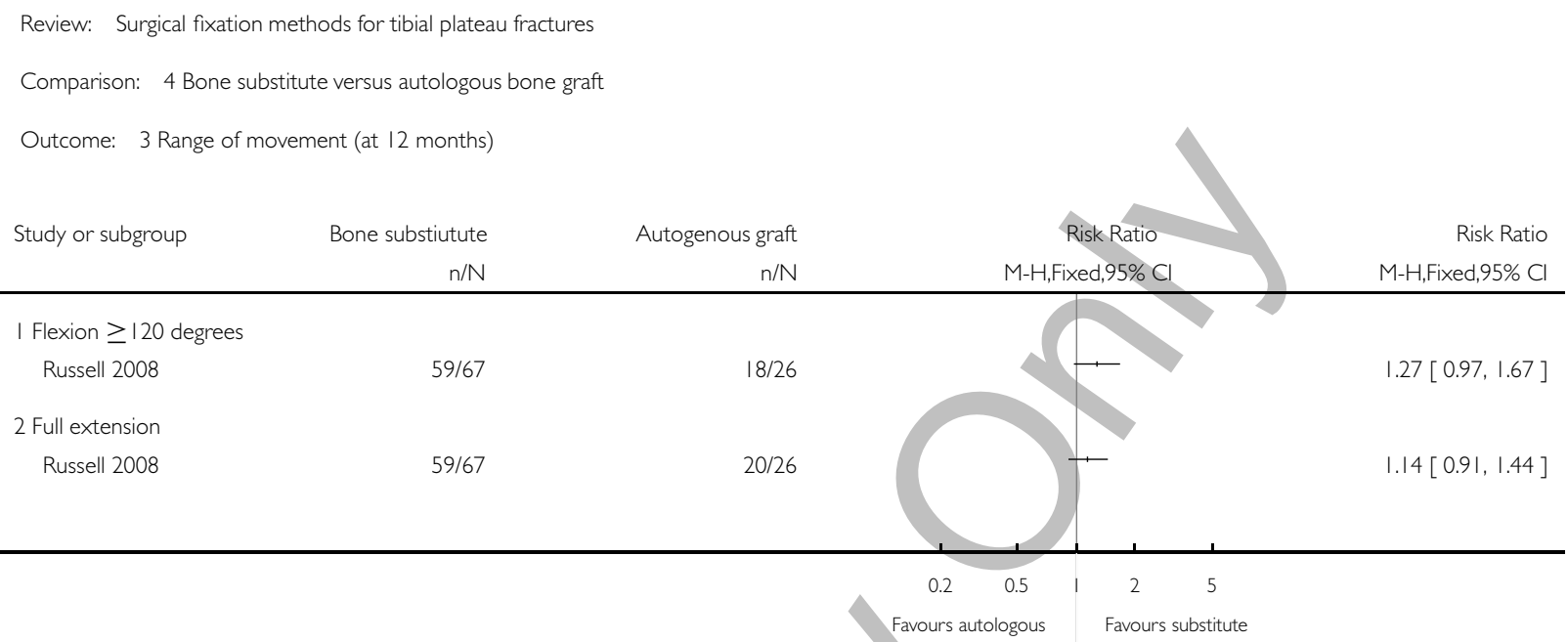

Analysis 4.4. Comparison 4 Bone substitute versus autologous bone graft, Outcome 4 Radiographic outcomes.

Review: Surgical fixation methods for tibial plateau fractures

Comparison: 4 Bone substitute versus autologous bone graft

Outcome: 4 Radiographic outcomes

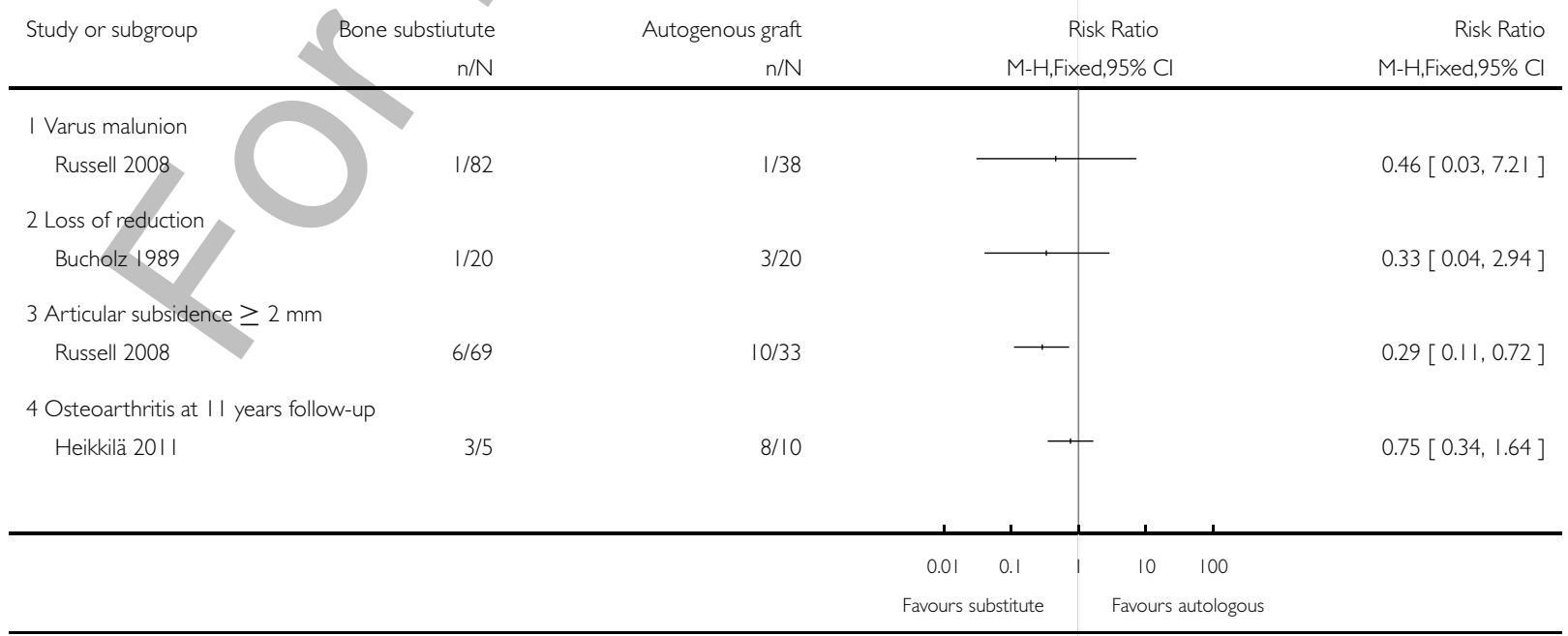

Surgical fixation methods for tibial plateau fractures (Review)

Copyright $\odot 2015$ The Cochrane Collaboration. Published by John Wiley \& Sons, Ltd. 


\section{Analysis 4.5. Comparison 4 Bone substitute versus autologous bone graft, Outcome 5 Anatomical}

malalignment.

Review: Surgical fixation methods for tibial plateau fractures

Comparison: 4 Bone substitute versus autologous bone graft

Outcome: 5 Anatomical malalignment

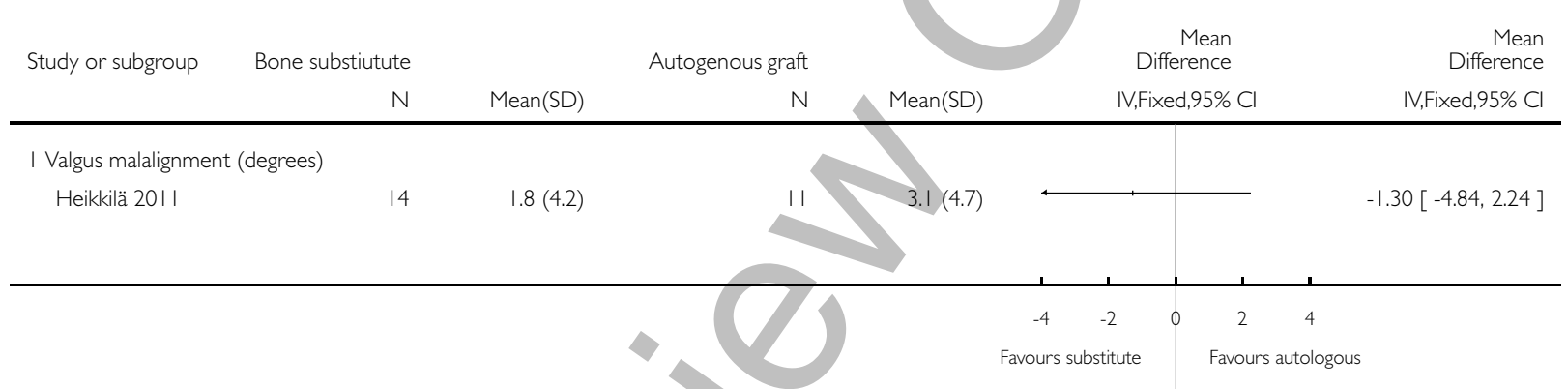

\section{A P P E N D I C E S}

\section{Appendix I. Search strategies}

\section{CENTRAL (Wiley Online Library)}

\#1 MeSH descriptor: [Tibial Fractures] this term only (239)

\#2 MeSH descriptor: [Tibia] this term only (394)

\#3 MeSH descriptor: [Knee] this term only (575)

\#4 MeSH descriptor: [Knee Injuries] this term only (630

\#5 MeSH descriptor: [Knee Joint] this term only (2170)

\#6 (tibia* or tibiofemoral):ti,ab,kw (2570)

$\# 7$ \#2 or \#3 or \#4 or \#5 or \#6 (5240)

\#8 MeSH descriptor: [Fractures, Bone] this term only (1196)

\#9 MeSH descriptor: [Fracture Healing] this term only (402)

\#10 (fracture or schatzker):ti,ab,kw (9803)

\#11 \#8 or \#9 or \#10 (9803)

\#12 \#7 and \#11 (658)

Surgical fixation methods for tibial plateau fractures (Review)

Copyright @ 2015 The Cochrane Collaboration. Published by John Wiley \& Sons, Ltd. 
\#13 \#1 or \#12 (658)

\#14 (plateau or intra*articular):ti,ab,kw (1881)

\#15 \#13 and \#14 (33)

\#16 MeSH descriptor: [Orthopedics] this term only (328)

\#17 MeSH descriptor: [Surgical Procedures, Operative] explode all trees (99550)

\#18 MeSH descriptor: [Orthopedic Fixation Devices] explode all trees (2053)

\#19 MeSH descriptor: [Fracture Fixation] explode all trees (1248)

\#20 (surg* or operat*):ti,ab,kw (107815)

\#21 (pin* or nail* or screw* or plate* or plating or fix* or ORIF or circular frame):ti,ab,kw (33050)

\#22 (\#16 or \#17 or \#18 or \#19 or \#20 or \#21) (180293)

$\# 23 \# 15$ and \#22 (33) [trials]

\section{MEDLINE (Ovid Online)}

1 Tibial Fractures/ (12616)

2 Tibia/ (27264)

3 Knee/ or Knee Injuries/ or Knee Joint/ (59976)

4 (tibia* or tibiofemoral).tw. (63038)

5 or/2-4 (121891)

6 Fractures, bone/ or Fracture Healing/ (55659)

7 fracture.tw. (112240)

8 schatzker.tw. (177)

9 or/6-8 (143023)

10 and/5,9 (11629)

11 or/1,9 (148610)

12 (plateau or intra?articular).tw. (36194)

13 and/11-12 (1984)

14 Orthopedics/ (16439)

15 exp Surgical Procedures, Operative/ (2437772)

16 exp Orthopedic Fixation Devices/ (61129)

17 exp Fracture Fixation/ (47611)

18 su.fs. (1611266)

19 (surg* $^{*}$ or operat*).tw. (1879733)

20 (pin* $^{*} 1$ or nail ${ }^{*}$ or screw* 1 or plate* 1 or plating or fix* or ORIF or circular frame).tw. (454447)

$21 \mathrm{or} / 14-20$ (4142916)

22 and/13,21 (1656)

23 Randomized controlled trial.pt. (388379)

24 Controlled clinical trial.pt. (89811)

25 randomized.ab. (308153)

26 placebo.ab. (159551)

27 Drug therapy.fs. (1743185)

28 randomly.ab. (222343)

29 trial.ab. (320966)

30 groups.ab. (1402804)

31 or/23-30 (3442434)

32 exp Animals/ not Humans/ (4009228)

3331 not 32 (2956547)

34 and/22,33 (206)

\section{EMBASE (Ovid Online)}

1 Tibia Fracture/ (11719)

2 Tibia/ (29063)

Surgical fixation methods for tibial plateau fractures (Review)

Copyright $\odot 2015$ The Cochrane Collaboration. Published by John Wiley \& Sons, Ltd. 
3 Knee/ or Knee injury/ (53649)

4 (tibia* or tibiofemoral).tw. (73821)

5 or/2-4 (126482)

6 Fracture/ or Fracture healing/ (80517)

7 fracture.tw. (133099)

8 schatzker.tw. (192)

96 or 7 or 8 (175596)

105 and $9(13825)$

111 or $9(180587)$

12 (plateau or intra?articular).tw. (41564)

1311 and $12(2222)$

14 Orthopedics/ (18310)

15 exp Orthopedic equipment/ (147468)

16 Fracture fixation/ (21355)

17 Surgery/ (270013)

18 (surg* or operat*).tw. (2344761)

19 (pin* 1 or nail $^{*}$ or screw* 1 or plate* 1 or plating or fix* or ORIF or circular frame).tw. (526705)

2014 or 15 or 16 or 17 or 18 or $19(2916651)$

2113 and 20 (1658)

22 Randomized controlled trial/ (351640)

23 Clinical trial/ (837981)

24 Controlled clinical trial/ (386732)

25 Randomization/ (63178)

26 Single blind procedure/ (18765)

27 Double blind procedure/ (117719)

28 Crossover procedure/ (40113)

29 Placebo/ (258051)

30 Prospective study/ (260827)

31 ((clinical or controlled or comparative or placebo or prospective* or randomi\#ed) adj3 (trial or study)).tw. (781821)

32 (random* adj7 (allocat* or allot* or assign* or basis* or divid* or order*)).tw. (189734)

33 ((singl* or doubl* or trebl* or tripl*) adj7 (blind* or mask*)).tw. (169251)

34 (cross?over* or (cross adj1 over*)).tw. (72386)

35 ((allocat* or allot* or assign* or divid*) adj3 (condition* or experiment* or intervention* or treatment* or therap* or control* or group $\left.\left.{ }^{*}\right)\right)$.tw. (250407)

36 RCT.tw. (14706)

37 or/22-36 (1951610)

38 Case Study/ or Abstract Report/ or Letter/ (946992)

3937 not 38 (1912317)

4021 and 39 (235)

\section{Open Grey}

1. Tibial (101)

\section{WHO International Clinical Trials Registry Platform}

1. Tibial Plateau (14)

\section{Current Controlled Trials}

1. Tibial (295)

Surgical fixation methods for tibial plateau fractures (Review) 


\section{United States National Institute of Health Trials Registry (clinicaltrials.gov)}

1. Tibial Plateau (15)

\section{Bone and Joint Journal Orthopaedic Proceedings}

Astract or title: Tibial Plateau

AND

Full text or abstract or title: Fracture (199)

\section{CONTRIBUTIONSOFAUTHORS}

Iain McNamara, Caroline Hing, Toby Smith, Allan Clark, Dominic Nielsen, Simon Donell and Karen Shepherd all contributed to the planning, design, and conduct of this review, and have reviewed and agreed the final review.

Iain McNamara is the guarantor of the protocol and the full review.

\section{DECLARATIONSOF INTEREST}

None known.

\section{SOURCES OF SUPPORT}

\section{Internal sources}

- Norfolk and Norwich University Hospital, Norwich, UK.

Computing, administration and library services (I McNamara, T Smith, S Donell)

- University of East Anglia, Norwich, UK.

Computing and administration services (T Smith, A Clark, S Donell).

- St George's Hospital, London, UK.

Computing services and clinical expertise (C Hing, D Neilsen)

\section{External sources}

- No sources of support supplied

\section{DIFFERENCES BETWEEN PROTOCOLANDREVIEW}

On reviewing the included papers and data extraction, the review team considered that intraoperative results (length of surgery, blood loss, length of incision) and return to pre-injury activity level were important outcome measures that should have been listed in the protocol. Hence these were added in as secondary outcome measures.

Risk of bias assessment for blinding of outcome assessment and completeness of outcome data was for all outcomes rather than split by patient-reported outcomes and objective outcomes, as originally planned. This is because we considered that the rating was unlikely to be affected by the type of outcome for these sorts of trials. 